\title{
ULTRAVIOLET AND INFRARED DIAGNOSTICS OF STAR FORMATION AND DUST IN NGC 7331
}

\author{
David A. Thilker, ${ }^{1}$ Samuel Boissier, ${ }^{2,3}$ Luciana Bianchi, ${ }^{1}$ Daniela Calzetti, ${ }^{4}$ Alessandro Boselli, ${ }^{3}$ Daniel A. Dale, ${ }^{5}$ \\ Mark Seibert, ${ }^{6}$ Robert Braun, ${ }^{7}$ Denis Burgarella, ${ }^{3}$ Armando Gil de Paz, ${ }^{2,8}$ George Helou, ${ }^{9}$ Fabian Walter, ${ }^{10}$ \\ R. C. Kennicutt, Jr., ${ }^{11}$ Barry F. Madore, ${ }^{2}$ D. Christopher Martin, ${ }^{6}$ Tom A. Barlow, ${ }^{6}$ Karl Forster, ${ }^{6}$ \\ Peter G. Friedman, ${ }^{6}$ Patrick Morrissey, ${ }^{6}$ Susan G. Neff, ${ }^{12}$ David Schiminovich, ${ }^{13}$ Todd Small, ${ }^{6}$ \\ Ted K. Wyder, ${ }^{6}$ José Donas, $^{3}$ Timothy M. Heckman, ${ }^{14}$ Young-Wook Lee, ${ }^{15}$ \\ Bruno Milliard, ${ }^{3}$ R. Michael Rich, ${ }^{16}$ Alex S. Szalay, ${ }^{14}$ \\ Barry Y. Welsh, ${ }^{17}$ and Sukyoung K. YI ${ }^{15}$ \\ Received 2006 July 17; accepted 2006 September 26
}

\begin{abstract}
We present images of NGC 7331 obtained with GALEX and Spitzer, tracing UV and IR signatures of star formation. NGC 7331's morphology at $8-850 \mu \mathrm{m}$ is dominated by a central dust ring. This structure is a vigorous site of star formation (hosting one-third of the present activity) but remains inconspicuous in our GALEX UV imagery. Radial profile analysis and photometry for discrete UV- and UV+IR-selected substructures indicate a decline in UV extinction with increasing galactocentric distance, although highly attenuated star-forming regions can be found throughout the disk. UV-optical surface brightness profiles suggest a recent birthrate parameter $\left(b_{8}\right)$ that is highest in the outer part of the disk, even though the local star formation intensity peaks in the ring. Bolometric luminosity and UV attenuation are correlated in substructures on $0.4 \mathrm{kpc}$ scales, with a relationship similar to that established for starburst galaxies. The distribution of substructures in $L(\mathrm{IR}) / L(\mathrm{FUV}), L_{\lambda}(\mathrm{FUV}) / L_{\lambda}(\mathrm{NUV})$ space suggests that the majority of the disk is best characterized by Milky Way-type dust, with the exception of sources in the star-forming ring. As found by Calzetti et al. in M51, the observed 8 and $24 \mu \mathrm{m}$ luminosity for substructures in NGC 7331 are correlated, showing a decline in $L_{\nu}(8 \mu \mathrm{m}) / L_{\nu}(24 \mu \mathrm{m})$ with increasing luminosity. We demonstrate the dependence of $L_{\nu}(8 \mu \mathrm{m}) /$ $L_{\nu}(24 \mu \mathrm{m})$ on the local extinction-corrected $\mathrm{H} \alpha$ surface brightness (hence current $\Sigma_{\mathrm{SFR}}$ ). A power law of slope 1.64 (1.87) accurately describes the Schmidt-law relation versus $\Sigma_{\mathrm{H}_{2}}\left(\Sigma_{\text {gas }}\right)$ for molecular-dominated environments. The same locations show no correlation between $\Sigma_{\mathrm{SFR}}$ and $\Sigma_{\mathrm{H}_{\mathrm{I}}}$. For atomic-dominated regions above an apparent local star formation threshold, we found a trend for increasing $\Sigma_{\mathrm{SFR}}$ at higher $\Sigma_{\mathrm{H}}$, although the Schmidt-law correlation with molecular-only surface density persists in areas dominated by atomic gas.
\end{abstract}

Subject headings: galaxies: evolution — galaxies: individual (NGC 7331) — infrared: galaxies — ultraviolet: galaxies

Online material: color figures

\section{INTRODUCTION}

The Infrared Astronomical Satellite (IRAS) revolutionized our awareness of the physical conditions under which star formation proceeds in the most prodigious star-forming galaxies (Soifer et al. 1987; Sanders et al. 1988). This pioneering work was followed by the Infrared Space Observatory (ISO), which provided benchmark surveys of normal galaxies (Dale et al. 2000; Tuffs et al. 2002; Bendo et al. 2002). Extragalactic sources having the highest infrared (IR) luminosity (and hence star formation rate [SFR]) are heavily attenuated at shorter wavelengths, evidently forming the bulk of their stars behind (and within) an obscuring veil of dust

\footnotetext{
1 Center for Astrophysical Sciences, The Johns Hopkins University, 3400 North Charles Street, Baltimore, MD 21218; dthilker@pha.jhu.edu.

2 Observatories of the Carnegie Institution of Washington, 813 Santa Barbara Street, Pasadena, CA 91101.

3 Laboratoire d'Astrophysique de Marseille, BP 8, Traverse du Siphon, 13376 Marseille Cedex 12, France.

4 Space Telescope Science Institute, 3700 San Martin Drive, Baltimore, MD 21218.

5 Department of Physics and Astronomy, University of Wyoming, Laramie, WY 82071

6 California Institute of Technology, MC 405-47, 1200 East California Boulevard, Pasadena, CA 91125.

7 ASTRON, P.O. Box 2, 7990 AA Dwingeloo, Netherlands.

8 Departamento de Astrofísica, Universidad Complutense de Madrid, Madrid 28040, Spain.
}

(Soifer et al. 1987; Sanders et al. 1988; Sanders \& Mirabel 1996; Calzetti 2001), although this does not preclude the existence of ultraviolet (UV)-luminous/IR-faint galaxies having very high SFRs (e.g., Burgarella et al. 2006; Hoopes et al. 2007). Could a similar relationship between SFR and dust attenuation hold on subgalactic scales, as anticipated on the basis of the Schmidt law (Calzetti et al. 2005)? Indeed, how much star formation in nearby, "well-known" galaxies remains largely or completely hidden due to our predominately optical view of the universe? When revealed with instruments such as the Spitzer Space Telescope, might this unrecognized star formation change our understanding of issues

\footnotetext{
9 California Institute of Technology, MC 314-6, Pasadena, CA 91101.

10 Max-Planck-Institut fur Astronomie, Konigstuhl 17, 69117 Heidelberg, Germany.

${ }^{11}$ Institute of Astronomy, University of Cambridge, Madingley Road, Cambridge CB3 0HA, UK.

12 Laboratory for Astronomy and Solar Physics, NASA Goddard Space Flight Center, Greenbelt, MD 20771.

13 Department of Astronomy, Columbia University, New York, NY 10027.

14 Department of Physics and Astronomy, The Johns Hopkins University, Homewood Campus, Baltimore, MD 21218.

15 Center for Space Astrophysics, Yonsei University, Seoul 120-749, Korea.

16 Department of Physics and Astronomy, University of California, Los Angeles, CA 90095.

17 Space Sciences Laboratory, University of California at Berkeley, 601 Campbell Hall, Berkeley, CA 94720.
} 
as fundamental as galaxy classification and evolution? Recent advances in the morphological classification of galaxies on the basis of how their evolved Population II component appears in near-IR imagery (e.g., Block \& Puerari 1999) suggest that dustenshrouded star formation may likewise be critical to our assessment of galaxy evolution.

Until recently, however, it has been difficult to obtain both IR and UV imagery of common targets having adequate resolution and sensitivity in each band to gauge the balance of "hidden" and unobscured star formation. Spitzer (Werner et al. 2004) and the Galaxy Evolution Explorer (GALEX; Martin et al. 2005) now routinely provide morphologically detailed IR and UV images of targets well beyond the Local Volume $(\sim 10 \mathrm{Mpc})$, permitting the study of a significant and representative sample of galaxies. In the present paper, we demonstrate the power of combining imagery from Spitzer and GALEX by taking a multiwavelength look at the nearby spiral NGC 7331, which was an early observation for the GALEX Nearby Galaxy Survey (NGS; Bianchi et al. 2003; Gil de Paz et al. 2006) and the Spitzer Infrared Nearby Galaxies Survey (SINGS; Kennicutt et al. 2003). We build on the analysis of two preceding SINGS studies. In particular, Regan et al. (2004) have already conducted analysis of the Spitzer Infrared Array Camera (IRAC) and Multiband Imaging Photometer for Spitzer (MIPS) data for NGC 7331, with ancillary H I, CO, and submillimeter data. Smith et al. (2004) presented Spitzer Infrared Spectrograph (IRS) observations for varied environments within NGC 7331, discovering a new dust emission feature at $17.1 \mu \mathrm{m}$. This spectral feature is thought to be a tracer of polycyclic aromatic hydrocarbons (PAHs), as are the well-known 7.7 and $11.3 \mu \mathrm{m}$ bands.

In several ways NGC 7331 bears similarity to the Milky Way. It is classified as a SA(s)b galaxy, whereas the Milky Way appears to be SAB(rs)bcII (de Vaucouleurs \& Pence 1978). NGC 7331 has a total stellar+gas mass of $\sim 1.6 \times 10^{11} M_{\odot}$ via the baryonic TullyFisher relation (McGaugh 2005) and a virial mass $>4 \times 10^{11} M_{\odot}$ (from the $\mathrm{H}$ I rotation curve). In comparison, the Milky Way has an estimated virial mass of $\sim 10^{12} M_{\odot}$ (Kravtsov et al. 2004). The gaseous content of NGC 7331 is dominated by a massive $\mathrm{H}$ I disk containing $M_{\mathrm{H}_{\mathrm{I}}} \sim 1.2 \times 10^{10} M_{\odot}$ (Braun et al. 2003$)$, whereas the most recent Galactic $\mathrm{H}_{\mathrm{I}}$ models suggest $3 \times 10^{9} M_{\odot}$ for the Milky Way (Nakanishi \& Sofue 2003). The molecular hydrogen content of NGC 7331 has been estimated as $M_{\mathrm{H}_{2}}=4.7 \times 10^{9} M_{\odot}$ using Berkeley-Illinois-Maryland Array (BIMA) Survey of Nearby Galaxies (SONG) observations (Helfer et al. 2003). Most of this material is confined to a molecular ring, which was earlier recognized by Young \& Scoville (1982). The existence of this ring further accentuates the similarity between NGC 7331 and the Milky Way. The Milky Way's 5 kpc ring hosts a large fraction of the known Galactic star formation activity. Very Large Array (VLA) radio continuum observations (Cowan et al. 1994; Filho et al. 2002) demonstrate that NGC 7331's ring contains numerous nonthermal sources that are probably supernova remnants. Star formation throughout NGC 7331 has been studied by Petit (1998) using the typical tracer of $\mathrm{H} \alpha$ emission from $\mathrm{H}$ II regions. Dutil \& Roy (1999) conducted an abundance study of 164 such nebulae, establishing a mild metallicity gradient in NGC 7331. It has been claimed that NGC 7331 contains a counterrotating bulge (Prada et al. 1996), but this conclusion was contested by Bottema (1999). No gas-rich ( $\mathrm{H}$ I-detected) companions are known within $0.5^{\circ}$ (125 kpc) and $400 \mathrm{~km} \mathrm{~s}^{-1}$ in redshift (Haynes et al. 1998).

Throughout this paper, we adopt the Cepheid distance of 14.7 Mpc (Freedman et al. 2001) to NGC $7331\left(1^{\prime \prime} \sim 71 \mathrm{pc}, 1 \mathrm{kpc} \sim\right.$ $\left.14^{\prime \prime}\right)$. We also assume a foreground screen of Galactic extinction resulting in $E(B-V)=0.091 \mathrm{mag}$ (Schlegel et al. 1998). In $\S 2$ we describe our space-based IR $(3.6-160 \mu \mathrm{m})$ and UV (FUV:
$1530 \AA$, NUV: $2310 \AA$ ) observations, new imaging in the $\mathrm{H} \alpha$ and $B V R I$ bands, new radio-continuum synthesis observations, and our corollary database. Section 3 gives an overview of the analysis conducted using this multiwavelength data set, including details regarding region photometry and radial profiles. Section 4 presents our results, including a comparison of UV-and IR-selected substructures in NGC 7331 on scales of $\sim 0.4 \mathrm{kpc}$. Global and radial measures are also given to place the discrete sources in context. Our discussion emphasizes the necessity of both UV and IR star formation tracers if a complete census of massive star formation over the past few hundred megayears is the goal, although even higher spatial resolution is needed to completely remove the degeneracy between reddening and aging for individual star-forming regions. We conclude with a brief summary in $\oint 5$.

\section{OBSERVATIONS}

The data set assembled for this study, although focused on Spitzer and GALEX observations, spans the radio, millimeter, submillimeter, far-IR (FIR), mid-IR (MIR), near-IR (NIR), visible, and UV bands. Our data set is summarized in Table 1 and shown in Figure 1.

\subsection{Spitzer Space Telescope Imaging}

Spitzer images of NGC 7331 were obtained with both the IRAC $(3.6,4.5,5.8$, and $8.0 \mu \mathrm{m})$ and $\operatorname{MIPS}(24,70$, and $160 \mu \mathrm{m})$ instruments as part of the SINGS Legacy project. The shortwavelength (3.6 and $4.5 \mu \mathrm{m}$ ) IRAC bands provide a view of the old stellar population nearly unhampered by extinction. The 8.0 $\mu \mathrm{m}$ IRAC band provides an excellent high-resolution diagnostic of the emission originating from PAHs, although one must first account for underlying stellar continuum. MIPS observations in the MIR and FIR regime are crucial for estimating the bolometric luminosity of constituent star-forming complexes, as they trace thermal emission from the reservoir of warm and cool dust irradiated by UV light from stellar clusters. A complete description of the observing strategy and data reduction can be found in Kennicutt et al. (2003) and Regan et al. (2004). IRAC and MIPS instrument descriptions are given by Fazio et al. (2004) and Rieke et al. (2004), respectively.

Each of the four IRAC images is a mosaic of two $4 \times 7$ grids, each covering a $12.5^{\prime} \times 15^{\prime}$ field. The total integrated exposure time is $240 \mathrm{~s}$ in the center of the field and $120 \mathrm{~s}$ at the edges (Regan et al. 2004). The SINGS IRAC pipeline was used to create the final mosaics, which exploits the subpixel dithering to better sample the emission. The measured point-spread functions (PSFs) have FWHM $\sim 2^{\prime \prime}(0.14 \mathrm{kpc})$ in all bands, although notable sidelobe differences exist. Sensitivity limits at $3 \sigma$ are $0.54,0.63,2.8$, and $3.5 \mu \mathrm{Jy} \operatorname{arcsec}^{-2}$ for the four bands, respectively.

The MIPS observations were obtained in scan-mapping mode covering a similar field of view as the IRAC observations, in two separate visits (to discriminate asteroids, the same strategy as for the IRAC observations). Total integration times were 160, 80, and $16 \mathrm{~s}$ at 24,70 , and $160 \mu \mathrm{m}$, respectively. The data were processed through the MIPS Instrument Team Data Analysis Tool (Gordon et al. 2005).

The NGC 7331 MIPS data were obtained early in the Spitzer mission, before adjustments were made to nominal bias voltage levels. As a result, at $70 \mu \mathrm{m}$, special corrections had to be made for latent images resulting from stim flashes. Because of the significantly longer wavelengths relative to IRAC, the MIPS mosaics PSFs have measured FWHM of $6.0^{\prime \prime}, \sim 18^{\prime \prime}$, and $\sim 40^{\prime \prime}$. Sensitivity limits at $3 \sigma$ are $4.2,35.8$, and $77.4 \mu \mathrm{Jy} \operatorname{arcsec}^{-2}$ for the three bands. 
TABLE 1

SumMary OF OBSERVATIONS

\begin{tabular}{|c|c|c|c|c|c|c|}
\hline \multirow[b]{2}{*}{ BAND } & \multirow[b]{2}{*}{ Telescope } & \multirow[b]{2}{*}{$\lambda_{c}$} & \multirow[b]{2}{*}{$\Delta_{\lambda}$} & \multicolumn{2}{|c|}{ Resolution } & \multirow[b]{2}{*}{ REFERENCE } \\
\hline & & & & $(\operatorname{arcsec})$ & $(\mathrm{kpc})$ & \\
\hline FUV ............... & $G A L E X$ & $1530 \AA$ & $1344-1786 \AA$ & 4.6 & 0.33 & This paper \\
\hline 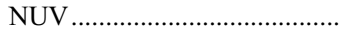 & $G A L E X$ & $2310 \AA$ & $1771-2831 \AA$ & 5.9 & 0.42 & This paper \\
\hline$B$ & KPNO $2.1 \mathrm{~m}$ & $4320 \AA$ & $1064 \AA$ & 1.5 & 0.11 & 1 \\
\hline$V \ldots \ldots \ldots \ldots \ldots$ & KPNO $2.1 \mathrm{~m}$ & $5395 \AA$ & $987 \AA$ & 1.5 & 0.11 & 1 \\
\hline$R$ & KPNO $2.1 \mathrm{~m}$ & $6471 \AA$ & $1444 \AA$ & 2.0 & 0.14 & 1 \\
\hline 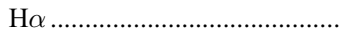 & KPNO $2.1 \mathrm{~m}$ & $6573 \AA$ & $67 \AA$ & 1.9 & 0.14 & 1 \\
\hline I & KPNO $2.1 \mathrm{~m}$ & $8212 \AA$ & $1922 \AA$ & 2.0 & 0.14 & 1 \\
\hline J & 2MASS & $1.25 \mu \mathrm{m}$ & $0.16 \mu \mathrm{m}$ & 2.5 & 0.18 & 2 \\
\hline H & 2MASS & $1.63 \mu \mathrm{m}$ & $0.30 \mu \mathrm{m}$ & 2.5 & 0.18 & 2 \\
\hline 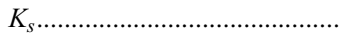 & 2MASS & $2.15 \mu \mathrm{m}$ & $0.31 \mu \mathrm{m}$ & 2.5 & 0.18 & 2 \\
\hline $3.6 \mu \mathrm{m}$ & Spitzer IRAC & $3.56 \mu \mathrm{m}$ & $0.75 \mu \mathrm{m}$ & 2.0 & 0.14 & 1 \\
\hline $4.5 \mu \mathrm{m}$ & Spitzer IRAC & $4.52 \mu \mathrm{m}$ & $1.01 \mu \mathrm{m}$ & 2.0 & 0.14 & 1 \\
\hline $5.8 \mu \mathrm{m}$ & Spitzer IRAC & $5.73 \mu \mathrm{m}$ & $1.42 \mu \mathrm{m}$ & 2.0 & 0.14 & 1 \\
\hline $8.0 \mu \mathrm{m}$ & Spitzer IRAC & $7.91 \mu \mathrm{m}$ & $2.93 \mu \mathrm{m}$ & 2.1 & 0.15 & 1 \\
\hline $24 \mu \mathrm{m}$ & Spitzer MIPS & $24 \mu \mathrm{m}$ & $5 \mu \mathrm{m}$ & 6 & 0.43 & 1 \\
\hline $70 \mu \mathrm{m}$ & Spitzer MIPS & $70 \mu \mathrm{m}$ & $19 \mu \mathrm{m}$ & 18 & 1.3 & 1 \\
\hline $160 \mu \mathrm{m}$ & Spitzer MIPS & $160 \mu \mathrm{m}$ & $35 \mu \mathrm{m}$ & 40 & 2.8 & 1 \\
\hline $450 \mu \mathrm{m}$ & JCMT SCUBA & $450 \mu \mathrm{m}$ & $50 \mu \mathrm{m}$ & 7.6 & 0.54 & 1,3 \\
\hline $850 \mu \mathrm{m}$ & JCMT SCUBA & $850 \mu \mathrm{m}$ & $80 \mu \mathrm{m}$ & 14.9 & 1.1 & 1,3 \\
\hline 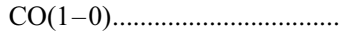 & BIMA & $2.6 \mathrm{~mm}$ & $\ldots$ & $6.1 \times 4.9$ & $0.43 \times 0.35$ & 1,4 \\
\hline 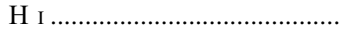 & VLA & $21 \mathrm{~cm}$ & $\cdots$ & $6.1 \times 5.6$ & $0.43 \times 0.40$ & 1 \\
\hline 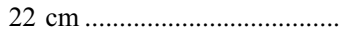 & WSRT & $22 \mathrm{~cm}$ & $\ldots$ & $22.6 \times 11.0$ & $1.6 \times 0.78$ & This paper \\
\hline
\end{tabular}

REFERENCES.-(1) Regan et al. 2004; (2) Jarrett et al. 2003; (3) Bianchi et al. 1998; (4) Helfer et al. 2003.

The resolution of the MIPS data limit the range of physical scales over which we can directly probe the spectral energy distribution (SED) within NGC 7331. Extending SED coverage from the UV to 24,70 , and $160 \mu \mathrm{m}$ implies limiting projected scales of $0.4,1.2$, and $2.7 \mathrm{kpc}$, respectively. However, in $\S 3.2$, we describe a technique for estimating the total IR luminosity of dust complexes using a combination of IRAC $8 \mu \mathrm{m}$ and MIPS $24 \mu \mathrm{m}$ observations (Calzetti et al. 2005). Following this method, we are able to compare the distribution of IR and UV flux on nearly identical scales.

\subsection{Galaxy Evolution Explorer \\ Far- and Near-Ultraviolet Imaging}

GALEX obtained far-UV (FUV; 1350-1750 ^) and near-UV (NUV; 1750-2750 $\AA$ ) direct imaging observations of NGC 7331 for a total duration of $3322 \mathrm{~s}$, spanning five visits during 2003 August. These data were pipeline processed and co-added to produce a single deep image in each UV band. The images analyzed herein are a product of pipeline version ops-4 .0-RC1 and were included in the first public GALEX data release (GR1). ${ }^{18}$ Details on the GALEX mission and on-orbit performance are provided by Martin et al. (2005) and Morrissey et al. (2005). The UV images primarily trace photospheric emission from massive stars.

The GALEX PSF is band and position dependent, although neither our FUV or NUV data are diffraction limited (as is the case for Spitzer). The GALEX FUV image of NGC 7331 is characterized by a typical PSF FWHM of $4.6^{\prime \prime}(0.33 \mathrm{kpc})$, with $80 \%$ of the point-source flux enclosed within $6^{\prime \prime}$. For the NUV band, the FWHM is $5.9^{\prime \prime}(0.42 \mathrm{kpc})$ with an $80 \%$ enclosed energy diameter of $8^{\prime \prime}$. In both bands the PSF varies with field position from center to edge. As the integrated FUV and NUV images are

\footnotetext{
${ }^{18}$ Available from the Multimission Archive at STScI (MAST) at http://galex .stsci.edu/GR1.
}

generated from photon-counting observations obtained while the spacecraft executes a compact spiral dither pattern, future pipeline improvements could yield modestly improved PSF shapes and sizes. Likewise, distortion corrections are made to the photon data in order to accurately register the distribution of observed counts with the sky. The IR1.0 pipeline based its distortion corrections on a preliminary set of in-flight calibration data, which successfully reduced astrometric errors for $80 \%$ of sources lying in the central degree of the GALEX field to less than $1.1^{\prime \prime}$.

The accuracy of GALEX photometric calibration is continually improving through a series of white dwarf observations to gauge the instrumental zero point, in addition to a program of on-orbit flat-field mapping. For GR1 data products, the photometric zero points appropriate to the FUV and NUV bands were determined using observations of the white dwarf LDS 749B. Uncertainty in the zero points is about 0.07 mag (P. Morrisey 2007, private communication). Residual scatter in the flat field is approximately $7 \%$. The rms noise in the NGC 7331 images is $\sim 9.8 \times$ $10^{-19} \mathrm{ergs} \mathrm{s}^{-1} \mathrm{~cm}^{-2} \AA^{-1}$ for FUV and $3.1 \times 10^{-18} \operatorname{ergs~s}^{-1} \mathrm{~cm}^{-2}$ $\AA^{-1}$ for NUV at the scale of the PSF.

\subsection{Ancillary Imaging}

The distribution of neutral atomic hydrogen in NGC 7331 was observed at the VLA as part of the H I Nearby Galaxy Survey (THINGS; Walter et al. 2005). These data will be described fully by F. Walter et al. (2008, in preparation). High-resolution NGC 7331 $\mathrm{H}$ I observations were obtained while the VLA was deployed in the B configuration on 2003 October 20 . These new observations were combined with archival $\mathrm{C}$ and $\mathrm{D}$ configuration visibility data. The high-resolution ( $6^{\prime \prime}, 0.4 \mathrm{kpc}$ FWHM ) map we use here is sensitive to inclination corrected $N(\mathrm{HI}) \sim 1.4 \times 10^{20} \mathrm{~cm}^{-2}$.

CO data from BIMA SONG (Regan et al. 2001; Helfer et al. 2003) are used as a tracer for the distribution of molecular gas. The $2.6 \mathrm{~mm}$ data set combines synthesis observations from 

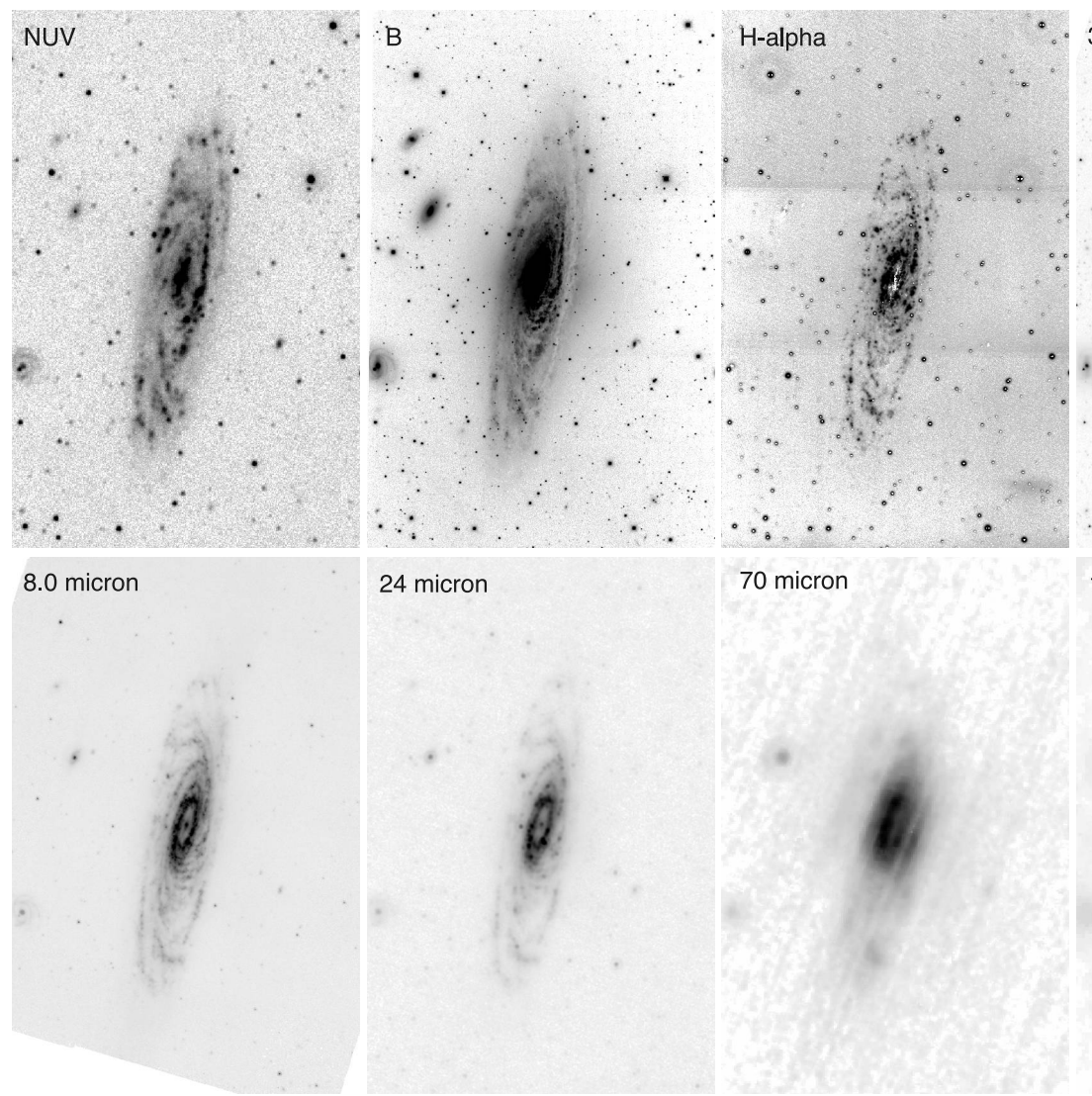

70 micron

450 micron

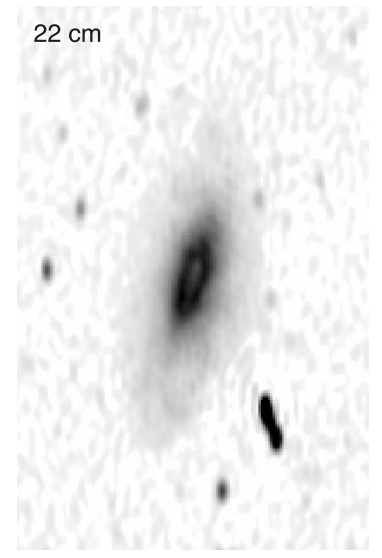

HI

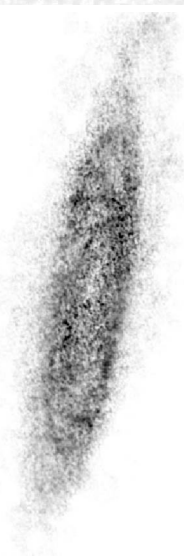

160 micron

3.6 micron

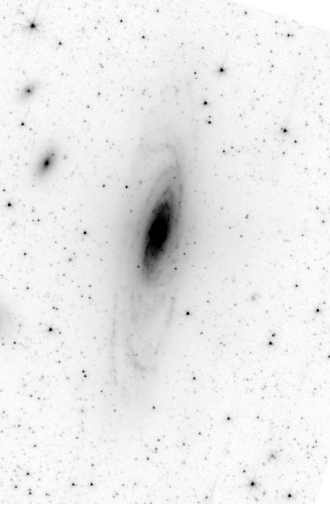

(1) mich

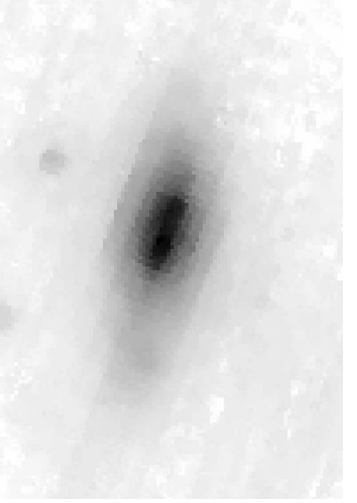

$\mathrm{CO}$

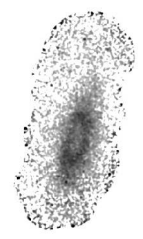

FIG. 1.- Multispectral morphology of NGC 7331. The GALEX NUV; KPNO $2.1 \mathrm{~m} B$ and continuum-subtracted H $\alpha$; Spitzer 3.6, 8, 24, 70, and 160; SCUBA 450 $\mu$ m; WSRT $22 \mathrm{~cm}$; VLA H I; and BIMA CO images presented here are displayed at their intrinsic resolution. With the exception of 70 and $160 \mu \mathrm{m}$, and $22 \mathrm{~cm}$, the PSF in each frame is comparable to or better than $6^{\prime \prime}(\sim 0.4 \mathrm{kpc})$. In order to maximize the useful dynamic range we have employed a square root transfer function for all panels except the $\mathrm{H}$ a and CO frames, which were scaled linearly. The field of view is $10.1^{\prime} \times 15.5^{\prime}$, corresponding to $43 \mathrm{kpc} \times 66 \mathrm{kpc}$ at the distance of NGC 7331. North is up and east is left. [See the electronic edition of the Supplement for a color version of this figure.]

BIMA and a total power map from the National Radio Astronomy Observatory (NRAO) $12 \mathrm{~m}$. An integrated CO image created from the combined data sets is sensitive to emission as faint as $3 \mathrm{Jy} \mathrm{beam}^{-1} \mathrm{~km} \mathrm{~s}^{-1}$ at $6^{\prime \prime}(0.4 \mathrm{kpc})$ resolution.

To trace the distribution of cold dust, we use 450 and $850 \mu \mathrm{m}$ James Clerk Maxwell Telescope (JCMT) Submillimetre Common User Bolometer Array (SCUBA) observations of NGC 7331, originally obtained by Bianchi et al. (1998) and Alton et al. (2001). The angular resolution of the SCUBA maps is $7.6^{\prime \prime}(0.5 \mathrm{kpc})$ and $14.9^{\prime \prime}$ (1 kpc) at 450 and $850 \mu \mathrm{m}$, respectively, superior to the limit of our comparable wavelength $(160 \mu \mathrm{m})$ Spitzer image.

IRAS observations of NGC 7331 at 12, 25, 60, and $100 \mu \mathrm{m}$ were obtained from the NASA/IPAC Infrared Science Archive (IRSA) for use in our analysis of radial profiles. They were pro- cessed via a HIRES request in order to obtain the best possible resolution.

A dedicated Westerbork Synthesis Radio Telescope (WSRT) survey of SINGS targets has been completed (Braun et al. 2007), providing a $22 \mathrm{~cm}$ radio continuum map for use in our study. The WSRT synthesis data at 18 and $22 \mathrm{~cm}$ for NGC 7331 were obtained on 2003 May 23. The synthesized beam has $22.6^{\prime \prime} \times$ $11.0^{\prime \prime}(1.6 \mathrm{kpc} \times 0.8 \mathrm{kpc}) \mathrm{FWHM}$, with a major axis position angle $0.4^{\circ}$ east of north. The rms noise in the cleaned image is $21 \mu \mathrm{Jy}$ beam $^{-1}$.

$\mathrm{H} \alpha$ observations of NGC 7331 were obtained using the Kitt Peak National Observatory (KPNO) $2.1 \mathrm{~m}$ on 2001 November 15. The contribution of continuum emission within the $67 \AA \mathrm{H} \alpha$ bandpass was estimated using an $R$-band observation acquired 
during the same run. This continuum image was scaled on the basis of foreground stars, prior to subtraction from the narrowband image. Our resulting line-only $\mathrm{H} \alpha$ image was flux calibrated using observations of spectrophotometric standard stars. Due to the $67 \AA$ bandpass of the narrowband filter, it transmitted the $\left[\mathrm{N}_{\text {III }}\right] \lambda \lambda 6548,6584$ doublet in addition to $\mathrm{H} \alpha$. Spectrophotometric measurements of Dutil \& Roy (1999, data kindly provided by Y. Dutil) show that $[\mathrm{N}$ II $] \lambda 6584 / \mathrm{H} \alpha$ varies between 0.2 and 0.7 in the $\mathrm{H}$ II region population of NGC 7331, with low values observed over all radii and higher values observed preferentially in $\mathrm{H}$ II regions closer to the galaxy center. Note that $[\mathrm{N} \mathrm{II}] \lambda 6584 / \mathrm{H} \alpha$ is known to be even higher yet ( $\sim 1.9$; Hoopes \& Walterbos 2003$)$ in diffuse ionized gas. We adopted $\left[\mathrm{N}_{\mathrm{II}}\right] \lambda 6584 / \mathrm{H} \alpha=0.5$ as our study focuses on star-forming regions rather than the diffuse medium. Furthermore, we assumed [ $\left.\mathrm{N}_{\text {II }}\right] \lambda 6548 /\left[\mathrm{N}_{\mathrm{II}}\right] \lambda 6584=$ 0.345 (Nussbaumer \& Rusca 1979). The observed flux in the continuum-subtracted image was adjusted downward to account for this level of total [ $\mathrm{N}$ II] contamination $(65 \%$ of $\mathrm{H} \alpha)$. The surface brightness sensitivity of the $\mathrm{H} \alpha$ data set yields an rms noise of $8.6 \times 10^{-18} \mathrm{ergs} \mathrm{s}^{-1} \mathrm{~cm}^{-2} \operatorname{arcsec}^{-2}$. At $5 \sigma$ significance, we can detect an $\mathrm{H}$ II region in NGC 7331 having $\log \left(L_{\mathrm{H} \alpha}\right)\left(\mathrm{ergs} \mathrm{s}^{-1}\right)=$ 36.6 , equivalent to the nebula produced by four B0 stars or a single O8 star. Broadband $B V I$ imaging was also obtained using the $2.1 \mathrm{~m}$ at KPNO. These data were flux calibrated using standard stars, achieving an accuracy of approximately $15 \%$.

\section{DATA ANALYSIS}

\subsection{Multiwavelength Galaxy Morphology}

Figure 1 presents a montage of our GALEX, Spitzer, $\mathrm{H} \alpha$-optical, SCUBA $450 \mu \mathrm{m}$, WSRT $22 \mathrm{~cm}$, VLA H i, and BIMA SONG $\mathrm{CO}(J=1-0)$ imagery for NGC 7331 . The data are presented at their intrinsic resolution (see Table 1). The morphology of NGC 7331 is typical of an SAb galaxy in the optical, NIR, and UV. In particular, the $3.6 \mu \mathrm{m}$ IRAC band displays a relatively featureless bulge and bright inner disk, surrounded by subdued spiral arm structures. The optical $(B)$ image presented in Figure 1 exhibits evident dust lanes appearing in silhouette against the near (west) side of the disk. The star-forming spiral arms in which much of this dust resides eventually dominate the galaxy morphology at UV wavelengths. However, the Spitzer 3.6-70 $\mu \mathrm{m}$ imagery reveals a rather different picture of recent star formation in NGC 7331. Most cleanly defined in the $24 \mu \mathrm{m}$ MIPS image, we note the presence of a dust-laden star-forming ring at radii between $30^{\prime \prime}-60^{\prime \prime}(2.1-4.3 \mathrm{kpc})$. The ring is virtually absent in the GALEX FUV and NUV images. Although we cannot distinguish the star-forming ring at $160 \mu \mathrm{m}$, comparison of the $160 \mu \mathrm{m}$ data with our $70 \mu \mathrm{m}$ image convolved to a common resolution shows that the requisite level of detail is lost. Indeed, observations with SCUBA at 450 and $850 \mu \mathrm{m}$ confirm that the ring morphology persists to longer wavelengths (Bianchi et al. 1998). NGC 7331's 3 kpc ring was first discovered by Young \& Scoville (1982) and Telesco et al. (1982) in CO maps. As we show in $\S 4.1$, this structure hosts a significant fraction of the current star formation in NGC 7331 yet remains inconspicuous in broadband optical imagery. In the UV, the only indication of the ring's star formation activity is very limited emission from the interior edge of the ring's far side.

Despite the substantial extinction toward star-forming sites within the ring, implied by the lack of a large-scale UV morphological signature, our $\mathrm{H} \alpha$ image reveals bright $\mathrm{H}$ II regions along the entire ring. This is not the only instance in NGC 7331 where $\mathrm{H} \alpha$ is detected without a UV counterpart. Additional cases appear to be highly extincted regions of star formation distributed in the disk but outside the ring. Furthermore, $\mathrm{H}$ II nebulae associated with one or a few massive stars can be detected in our $\mathrm{H} \alpha$ image, whereas the GALEX data can only achieve this in the outer disk where resolution and crowding issues are less severe. These considerations demonstrate that $\mathrm{H} \alpha$ imaging provides a more complete view of current (approximately zero-age) star formation activity than GALEX, principally due to the conversion of Lyman continuum flux to Balmer series photons, which are less obscured than the nonionizing UV emission. The converse is true regarding recent star formation activity. On the near side (west) of NGC 7331, a UV-bright spiral arm segment (bisected by the minor axis) stretches continuously in a north-south direction. In the $\mathrm{H} \alpha$ image this structure is more patchy in appearance. Indeed, many locations of bright UV yet faint $\mathrm{H} \alpha$ emission are evident throughout the galaxy. We interpret these UV sources as complexes which, although moderately young, have not formed $\mathrm{O}$ stars in the past several megayears.

Figure 2 presents a side-by-side comparison of the multiband Spitzer and GALEX imagery. On the left is a false-color image showing a subset of the IRAC and MIPS data. For this figure, we assigned $24,8.0$, and $3.6 \mu \mathrm{m}$ to red, green, and blue channels, respectively. NGC 7331's $3 \mathrm{kpc}$ star-forming ring is the dominant feature in this representation. On the right, we present our GALEX UV imagery with NUV in red, the energy-weighted average of NUV and FUV in green, and FUV in blue. Color variations in the composite GALEX image reflect the net effect of changes in luminosity-weighted age among stellar complexes and a rather significant component of modulation by extinction. Note the highly reddened color of the inner disk.

In Figure 3 (left), we combine the Spitzer and GALEX data in a single color-composite image to illustrate the distribution of overall bolometric output from star formation in NGC 7331. We have assigned color channels as follows: red, scaled $F(24 \mu \mathrm{m})$; green, average of scaled $F(24 \mu \mathrm{m})$ and $F($ FUV $)$; and blue, $F($ FUV $)$. The $F(24 \mu \mathrm{m})$ was scaled by a constant factor of 11.5 to approximate the total IR flux (see $\S 3.2$ for justification). In the rendition of Figure 3 (left) we have preserved color information at all but the highest intensities, by applying a gamma correction independently to the luminance. Regions appearing orange or yellow are generally rather extincted locations of star formation, in which nearly all the UV radiation from young stars is absorbed by dust and reemitted in the IR. Some of the interarm gaps, with dust heated by clusters in neighboring spiral arms or the general interstellar radiation field, also appear orange-yellow. Environments showing up as shades of light gray in Figure 3 (left) demonstrate a more substantial contribution from UV light either (1) escaping dusty natal sites of cluster formation or (2) originating from more evolved stellar complexes positioned along or near the line of sight to the dust cloud(s). Finally, the substructures appearing blue correspond to evolved complexes (still young enough to have many surviving B stars) in which massive stellar content has appreciably dispersed outside the veil of dust. It should be emphasized that, although the image in Figure 3 (left) shows a clear trend for a higher incidence of blue structures in the outer portion of the disk (roughly $>0.5 R_{25}$ ) ${ }^{19}$ and away from the $3 \mathrm{kpc}$ dust ring, most sources appearing blue in this representation are still radiating strongly in the IR. The majority of stellar complexes in NGC 7331 are IR-dominated, with total IR $(3-1100 \mu \mathrm{m})$ luminosity exceeding the UV output by typical factors ranging from a few to several hundred. Even for a UV-selected sample of complexes, this remains true, as we show in $\S 4.3$. Independent of the

\footnotetext{
19 The quantity $R_{25}$ is defined as one-half of $D_{25}$ : the galaxy diameter in blue light evaluated at a surface brightness of $25 \mathrm{mag} \operatorname{arcsec}^{-2}$.
} 

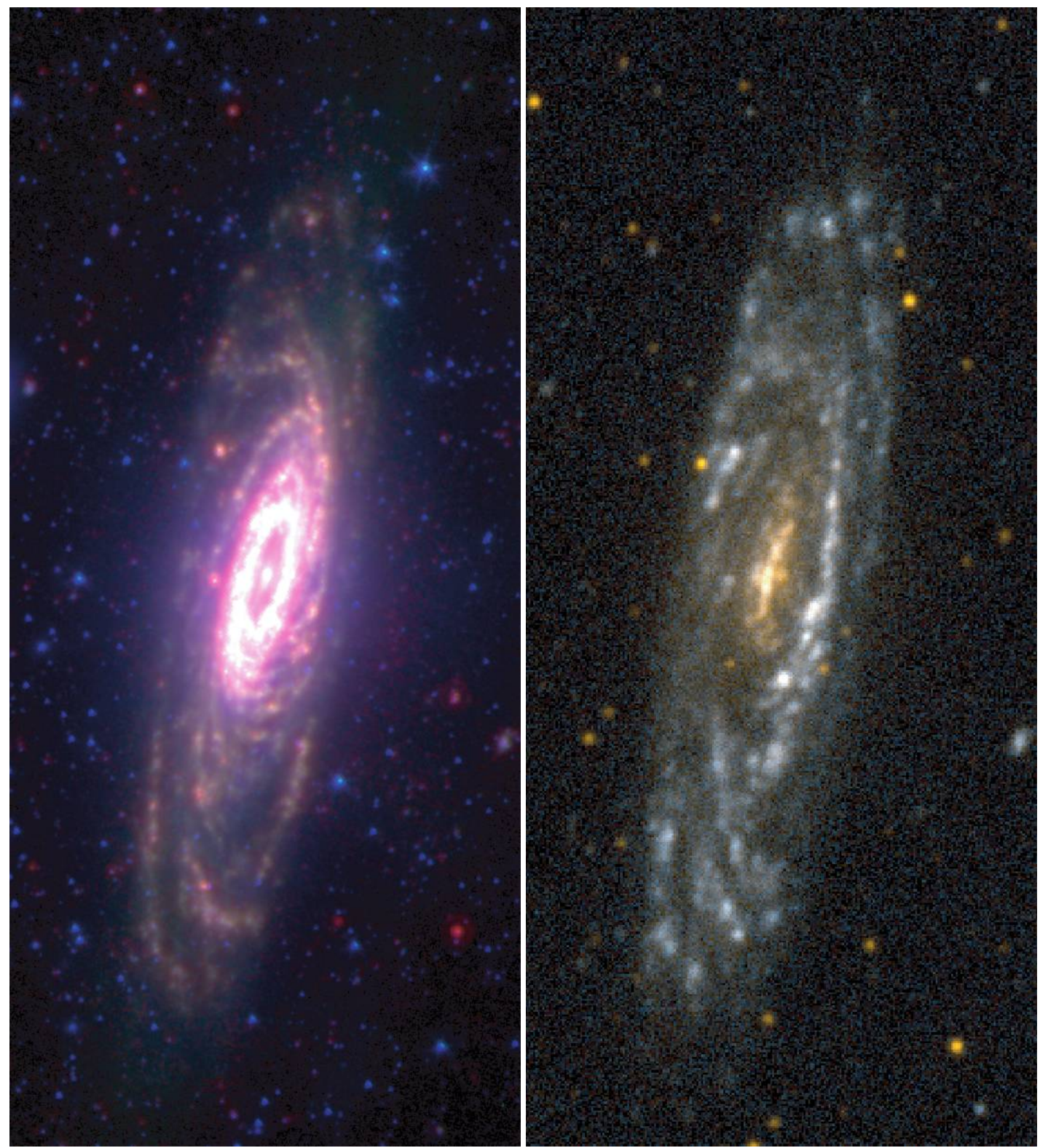

FIG. 2.-Comparison of Spitzer and GALEX observations of NGC 7331. Left: Spitzer color composite image generated from the $3.6 \mu \mathrm{m}(\mathrm{blue}), 8.0 \mu \mathrm{m}$ (green), and 24 $\mu \mathrm{m}$ (red) maps. The stellar continuum has not been subtracted from the IRAC $8 \mu \mathrm{m}$ data for this figure. Right: GALEX color image prepared using FUV (blue), NUV (red), and their energy-weighted average (green). The striking differences in IR and UV morphology are discussed in the text. NGC 7331's dusty star-forming ring dominates the Spitzer imagery yet is hardly noticeable in the GALEX data. The extent of the region depicted is $5.8^{\prime} \times 12.8^{\prime}$, or $25 \mathrm{kpc} \times 55 \mathrm{kpc}$ at the distance of NGC 7331 . North is up and east is left.

star-forming regions emphasized in Figure 3 (left), one can also see a substantial component of diffuse $24 \mu \mathrm{m}$ emission projected outside of the disk, especially on the near side (to the west) of NGC 7331. We suggest that the dust responsible for this emission could reside out of the plane, as it is not seen at the majority of position angles, after having been transported vertically by the action of the vigorous star formation in the ring (e.g., compare to M82; Hoopes et al. 2005; Engelbracht et al. 2006). Indeed, the extension of the out-of-plane emission along the galaxy major axis only reaches to about the size of NGC 7331's ring. An alternative explanation is that the $24 \mu \mathrm{m}$ emission is associated with dust formed in the atmospheres of asymptotic giant branch (AGB) stars, as seen in the Sombrero Galaxy (Bendo et al. 2006).

In order to constrain the dust emission within NGC 7331 on scales smaller than the $0.4 \mathrm{kpc}\left(6^{\prime \prime}\right)$ limit of our $24 \mu \mathrm{m}$ MIPS data, we turn to higher resolution $\left(0.15 \mathrm{kpc}, 2.1^{\prime \prime}\right) 8.0 \mu \mathrm{m}$ IRAC data. But emission from the population of cool stars remains appreciable at this wavelength, so we constructed a model for the stellar contribution to the observed $8.0 \mu \mathrm{m}$ IRAC image (see Appendix A). This model image, derived from the 3.6 and $4.5 \mu \mathrm{m}$ IRAC data, was subtracted from the $8.0 \mu \mathrm{m}$ frame to produce an image of "nonstellar" emission. It traces the distribution of PAH carriers, modulo their pumping and destruction (photoevaporation) mechanisms, with a secondary contribution from hot dust grains. Section 4.4.2 provides further detail on caveats related to interpretation of $8 \mu \mathrm{m}$ emission as a dust tracer. Nevertheless, we specifically sought to compare the $0.15 \mathrm{kpc}\left(2.1^{\prime \prime}\right)$-resolution dust distribution with other interstellar medium (ISM) constituents, such as neutral and ionized hydrogen.

Color channels in Figure 3 (center) are assigned as follows: red, $\mathrm{H} \alpha$; green, $8 \mu \mathrm{m}$ dust/PAHs; and blue, $N(\mathrm{H} \mathrm{I})$. The $\mathrm{H} \alpha$ and $8 \mu \mathrm{m}$ images are presented at $1.9^{\prime \prime}$ and $2.1^{\prime \prime}$ resolution $(\sim 0.15 \mathrm{kpc})$, 

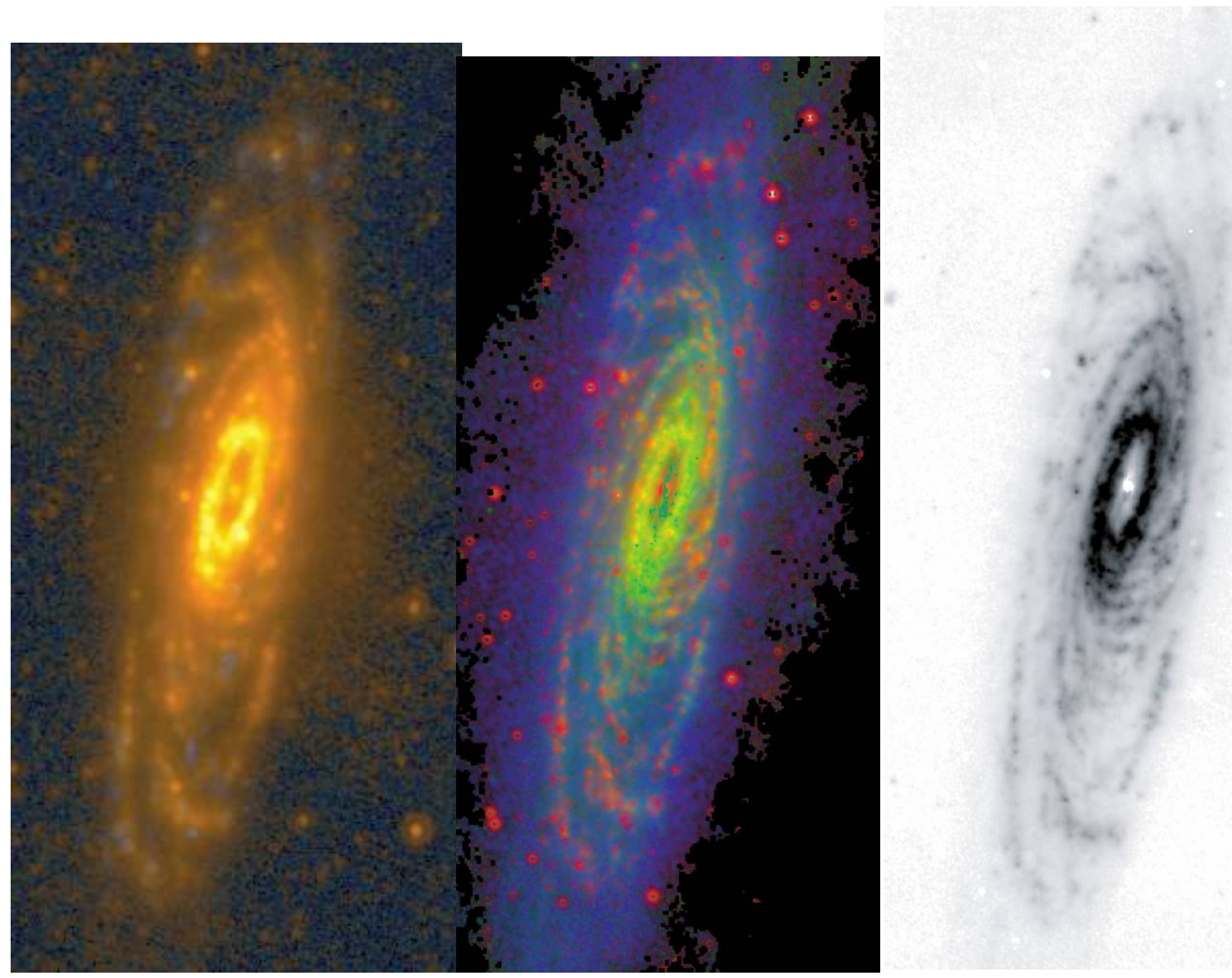

FIG. 3.-Left: Spitzer (MIPS) $24 \mu \mathrm{m}$, and GALEX NUV and FUV imaging of NGC 7331, presented as a color-composite image in which $24 \mu \mathrm{m}$ dust emission has been displayed in red, NUV emission in green, and FUV emission in blue. The field of view is $5.5 \times 10.8^{\prime}$. Note the complementary nature of the GALEX and Spitzer observations. Both IR and UV-dominated sources can be identified. The Spitzer $24 \mu \mathrm{m}$ image particularly highlights heavily attenuated star-forming regions, although our SINGS observations are deep enough to detect many sources for which the majority of bolometric luminosity emerges in the UV. GALEX observations are well suited for probing the young stellar content of locales forming stars at comparatively low rates and for gauging the effective attenuation toward starbursting regions. Middle: $\mathrm{H} \alpha$, PAH, and $\mathrm{H}$ I imaging of NGC 7331, presented as a color-composite image in which $\mathrm{H} \alpha$ emission has been displayed in red, PAH emission in green, and $\mathrm{H}$ I emission in blue. The field of view is the same as in left and right panels. Stellar residuals from the $\mathrm{H} \alpha$ continuum-subtraction process have not been masked, so as to provide a positional reference frame within the galaxy enabling comparison with broadband optical data. It appears the edge of the $\mathrm{H}$ I disk may be less populated with star-forming regions. Right: PAH emission in NGC 7331 at a spatial resolution of $\sim 0.15 \mathrm{kpc}$. The figure presents continuum-subtracted $8 \mu \mathrm{m}$ IRAC imaging of the same field shown in left and middle panels. The distribution of PAH emission breaks up into clumps typically arranged along NGC 7331's spiral arms and inner star-forming ring. Diffuse PAH emission fills the interarm gaps.

respectively, compared to $\sim 6^{\prime \prime}(0.4 \mathrm{kpc})$ for $\mathrm{H}$ I. We note many areas in the vicinity of the star-forming ring that exhibit diffuse PAH emission (appearing green or blue-green) amid an interspersed ensemble of $\mathrm{H}$ II regions and PAH clumps. In addition, the southern end of the ring is lacking $\mathrm{H}$ II regions yet is characterized by strong $8 \mu \mathrm{m}$ PAH emission. A lack of $\mathrm{H}$ in regions in the outer parts of the $\mathrm{H}$ I disk (with lowest column density) is apparent, perhaps reflecting the influence of star formation thresholds.

Figure 3 (right) shows the $8 \mu \mathrm{m}$ dust-only image in gray-scale form, to better illustrate the observed low-level PAH emission. This rendition highlights the morphology of diffuse PAHs mentioned above. Furthermore, the characteristic size and clumpiness of the $8 \mu \mathrm{m}$ peaks can be discerned as our highest resolution $(\sim 0.15 \mathrm{kpc})$ extinction-free tracer of star formation. The features observed as small, unresolved clumps in Figure 3 (right) are likely the dust counterparts of individual giant molecular clouds (GMCs), which have produced one or more stellar clusters.

\subsection{The Total Infrared Flux Distribution at GALEX Resolution}

Although Spitzer provides an unprecedentedly high-resolution view of NGC 7331's NIR-FIR morphology at the bandpasses sampled by IRAC and MIPS, the two longest wavelength images (70 and $160 \mu \mathrm{m}$ ) have diffraction-limited PSFs of 18 and $40^{\prime \prime}(1.3$ and $2.8 \mathrm{kpc}$ ) FWHM, respectively. Such resolution is substantially lower than that achieved in the UV by GALEX $\left(5-6^{\prime \prime}, \sim 0.4 \mathrm{kpc}\right)$. The $24 \mu \mathrm{m}$ MIPS image is also diffraction limited but has angular resolution comparable to our GALEX data.

Calzetti et al. (2005) showed that a correlation between $\log [L(\mathrm{IR}) / L(24 \mu \mathrm{m})]$ and $\log \left[L_{\nu}(8 \mu \mathrm{m}) / L_{\nu}(24 \mu \mathrm{m})\right]$ persists on subgalactic scales in M51, similar to the established relation applicable to entire star-forming galaxies (Dale \& Helou 2002, hereafter DH02). Calzetti et al. (2005) represented the correlation between $\log [L(\mathrm{IR}) / L(24 \mu \mathrm{m})]$ and $\log \left[L_{\nu}(8 \mu \mathrm{m}) / L_{\nu}(24 \mu \mathrm{m})\right]$ with a linear fit and used this result to estimate the total IR luminosity, $L(\mathrm{IR})$, of dust complexes evident on $6^{\prime \prime}$ scales using 
only the 8 and $24 \mu \mathrm{m}$ imagery. The factor of 7 spatial resolution improvement provided by this procedure almost certainly outweighs any possible errors introduced by unrecognized variation in the SED shape (at scales too fine for 70 and $160 \mu \mathrm{m}$ ). However, the constants of the Calzetti et al. (2005) calibration (their eq. [1]) were measured using large $(2.8 \mathrm{kpc})$ apertures, much larger than the $0.4 \mathrm{kpc}$ scale on which we are able to study the $24 \mu \mathrm{m}$ distribution in NGC 7331.

To ascertain calibration of the $\log [L(\mathrm{IR}) / L(24 \mu \mathrm{m})]$ versus $\log \left[L_{\nu}(8 \mu \mathrm{m}) / L_{\nu}(24 \mu \mathrm{m})\right]$ relation on scales directly comparable to the size of star-forming complexes and the GMCs within which they form, we redetermined the correlation on the basis of IR-selected clumps in the IRAC and MIPS imagery of M33 (courtesy B. Gehrz and the GGTOP team). Following Calzetti et al. (2005), we computed L(IR) from the DH02 Spitzer-specific formulation. Our M33 analysis will be the subject of a future paper, but equation (1) presents the updated prediction for $F(\mathrm{IR}) / F(24 \mu \mathrm{m})$ as a function of $F_{\nu}(8 \mu \mathrm{m}) / F_{\nu}(24 \mu \mathrm{m})$ at $160 \mathrm{pc}$ scales:

$$
\log \left[\frac{F(\mathrm{IR})}{F(24 \mu \mathrm{m})}\right]=1.06+0.475 \log \left[\frac{F_{\nu}(8 \mu \mathrm{m})}{F_{\nu}(24 \mu \mathrm{m})}\right] .
$$

Our result is intermediate between the Calzetti et al. (2005) scaling relation and the models of DH02. This is perhaps not surprising given that the $F(24 \mu \mathrm{m}) / F(\mathrm{IR})$ ratio of Calzetti et al. was 0.2 dex higher than $\mathrm{DH} 02$ at constant $F_{\nu}(8 \mu \mathrm{m}) / F_{\nu}(24 \mu \mathrm{m})$. Our equation (1) also yields $F(24 \mu \mathrm{m}) / F(\mathrm{IR})$ greater than DH02, but as Calzetti et al. describe, a shift in this direction is anticipated due to the greater importance of star-forming regions (vs. cirrus) in small apertures.

To use equation (1) in NGC 7331, we first convolved the IRAC $8 \mu \mathrm{m}$ dust-only image described in $\S 3.1$ (also see Fig. 3, right) with a kernel designed to degrade the $8 \mu \mathrm{m}$ PSF until it precisely matched the MIPS $24 \mu \mathrm{m}$ PSF (K. D. Gordon et al. 2008, in preparation). After resolution matching, the ratio of flux densities was computed for all positions, determining the degree of local deviation from $F($ IR $) / F(24 \mu \mathrm{m})=11.5$ as expected for $F_{\nu}(8 \mu \mathrm{m})=F_{\nu}(24 \mu \mathrm{m})$. On smallest scales probed by our $24 \mu \mathrm{m}$ image of NGC 7331, the logarithmic term in equation (1) generally varied between 0.0 and 0.5 , implying slightly less than a factor of 2 increase in $F(\mathrm{IR}) / F(24 \mu \mathrm{m})$ at positions characterized by relatively strong $8 \mu \mathrm{m}$ PAH emission (vs. $24 \mu \mathrm{m}$ ). The observed $24 \mu \mathrm{m}$ image was then scaled using equation (1) with our positiondependent information regarding $\log \left[F_{\nu}(8 \mu \mathrm{m}) / F_{\nu}(24 \mu \mathrm{m})\right]$. Finally, the total IR image (characterized by the $24 \mu \mathrm{m}$ PSF) was deconvolved using 25 iterations of a maximum entropy method algorithm. As expected for maximum entropy deconvolution, resolution of the output image is mildly variable but is comparable to the input PSF (without sidelobes).

\subsection{Adopted Star Formation Rate Formulations}

Both Spitzer and GALEX clearly reveal sites of recent or ongoing star formation, but the particular view afforded by each observatory actually reflects a juxtaposition of dissimilar locations (in terms of dust content) and temporal phases occurring within a star-forming environment. Individually, neither of the space-based data sets provides a complete census of locales which have been active in the recent past.

Excepting a possible contribution from cirrus heated by field stars, the IR luminosity traces present-day star formation taking place in dusty environments. Depending on the precise grain properties and the relative geometry of dust and stars, a variable fraction of the intrinsic UV luminosity from newly formed, embedded stellar clusters can escape without being absorbed and reprocessed into the IR. The observed UV emission may also include radiation arising from slightly more evolved populations in the same (structurally complicated) star-forming environment-locations still young in the sense of having a B star population, but old enough for such stars to have dispersed from within the bulk of dust. However, the IR and UV data jointly recover most of the bolometric luminosity, $L$ (bol), from young stellar clusters and starforming regions regardless of environment. Consequently, we estimate the bolometric luminosity originating from such stellar populations,

$$
L(\mathrm{bol}) \approx \nu_{\mathrm{FUV}} L_{\nu}(\mathrm{FUV})+(1-\eta) L(\mathrm{IR}),
$$

where $L_{\nu}(\mathrm{FUV})$ is only corrected for Milky Way foreground dust and $L(\mathrm{IR})$ is the total 3-1100 $\mu \mathrm{m}$ MIR-FIR luminosity computed directly with the $\mathrm{DH} 02$ relation or estimated from IRAC and MIPS data on small scales as described in $\S 3.2$. The $(1-\eta)$ term corrects for the fraction of IR luminosity associated with heating from old stars, $\eta$. The effective frequency of the GALEX FUV band is given by $\nu_{\mathrm{FUV}}$.

An empirical (e.g., Buat \& Xu 1996) or theoretical (e.g., Kennicutt 1983 , 1998b) conversion factor is still required to derive the SFR from the observed luminosity. Model-based calibrations of the SFR as a function of measured strength of the stellar continuum, nebular emission lines, thermal dust emission, and radio continuum are most commonly used. Kennicutt (1998b) provides a definitive review pertaining to the topic, carefully exposing the limitations encountered at each wavelength range. Hirashita et al. (2003) further developed a framework to establish consistency between multiple SFR tracers, accounting for issues such as the $\eta$-parameter, Lyman continuum leakage, and extinction. In Appendix B, we state the various calibrations used in our paper, which are based on Starburst99 (Leitherer et al. 1999) populationsynthesis models.

\subsection{Photometry}

Our multiwavelength database enables measurement of the observed SED at high spatial resolution across the disk of NGC 7331. Our photometry goals were to provide estimates of integrated flux in all bands for discrete stellar clusters and dust complexes, while also sampling the entire range of diverse physical conditions encountered in the galaxy (e.g., arm vs. interarm).

The first goal was addressed by conducting multiwavelength photometry of discrete substructures evident in NGC 7331. Two samples were identified on the basis of different "selection" images, specifically FUV and our bolometric (FUV+IR) representation. These images both have $\sim 6^{\prime \prime}(0.4 \mathrm{kpc})$ resolution. Note that the two samples are complementary yet not entirely independent. That is, a single stellar complex with associated dust emission can appear in both samples, but there are also some UV-selected clusters with no IR-selected counterpart and vice versa. Both samples are retained with such duplication throughout our photometry procedure and analysis, as this allows uncomplicated interpretation of wavelength-dependent selection effects. Substructures were detected and measured on the UV and UV+IR selection images using MMAP (D. Thilker et al. 2008, in preparation), which is a multiscale, multiwavelength successor to the adaptive photometry program HIIphot (Thilker et al. 2000). In order to morphologically distinguish small-scale substructures from slowly varying galactic emission we subtracted a background surface evaluated using a circular median operator of diameter $40^{\prime \prime}$ $(2.8 \mathrm{kpc})$. Sources exhibiting significant small-scale emission were identified on the background-subtracted image, which was 
TABLE 2

Рhotometry of UV+IR and UV-selected Substructures in NGC 7331

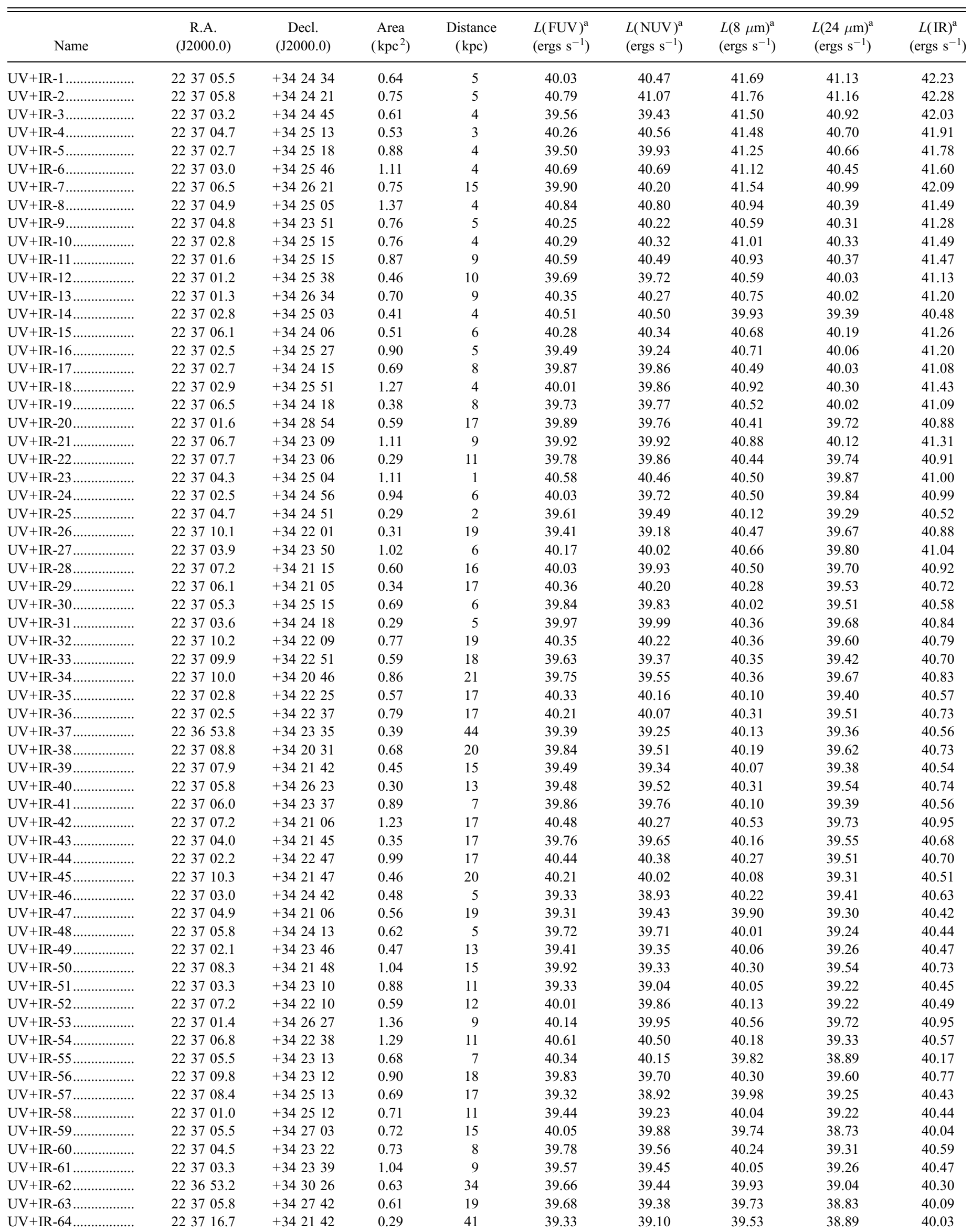


TABLE 2-Continued

\begin{tabular}{|c|c|c|c|c|c|c|c|c|c|}
\hline Name & $\begin{array}{c}\text { R.A. } \\
(\mathrm{J} 2000.0)\end{array}$ & $\begin{array}{c}\text { Decl. } \\
(\mathrm{J} 2000.0)\end{array}$ & $\begin{array}{c}\text { Area } \\
\left(\mathrm{kpc}^{2}\right)\end{array}$ & $\begin{array}{l}\text { Distance } \\
(\mathrm{kpc})\end{array}$ & $\begin{array}{l}L(\mathrm{FUV})^{\mathrm{a}} \\
\left(\mathrm{ergs} \mathrm{s}^{-1}\right)\end{array}$ & $\begin{array}{l}L(\mathrm{NUV})^{\mathrm{a}} \\
\left(\mathrm{ergs} \mathrm{s}^{-1}\right)\end{array}$ & $\begin{array}{l}L(8 \mu \mathrm{m})^{\mathrm{a}} \\
\left(\mathrm{ergs} \mathrm{s}^{-1}\right)\end{array}$ & $\begin{array}{l}L(24 \mu \mathrm{m})^{\mathrm{a}} \\
\left(\operatorname{ergs~s}^{-1}\right)\end{array}$ & $\begin{array}{c}L(\mathrm{IR})^{\mathrm{a}} \\
\left(\mathrm{ergs} \mathrm{s}^{-1}\right)\end{array}$ \\
\hline UV+IR-65 ............... & 223702.7 & +342730 & 0.49 & 11 & 40.43 & 40.27 & 39.45 & 38.77 & 39.93 \\
\hline UV+IR-66 ............... & 223706.4 & +342552 & 0.42 & 13 & 40.42 & 40.28 & 39.65 & 38.71 & 39.99 \\
\hline UV+IR-67 ................ & 223700.6 & +341826 & 0.51 & 44 & 39.50 & 38.79 & 39.82 & 38.85 & 40.15 \\
\hline UV+IR-68 ................ & 223709.1 & +342022 & 0.61 & 20 & 39.92 & 39.80 & 39.88 & 39.04 & 40.27 \\
\hline UV+IR-69 .............. & 223705.8 & +342754 & 0.53 & 20 & 39.51 & 39.54 & 39.41 & 38.71 & 39.88 \\
\hline UV+IR-70 ............... & 223714.0 & +342721 & 0.63 & 47 & 39.89 & 39.83 & 39.90 & 39.11 & 40.32 \\
\hline UV+IR-71 ................ & 223703.8 & +342601 & 0.35 & 5 & 39.41 & 39.04 & 39.98 & 39.52 & 40.58 \\
\hline UV+IR-72 ............... & 223701.4 & +342417 & 0.74 & 13 & 40.52 & 40.55 & 39.50 & 38.80 & 39.97 \\
\hline UV+IR-73 .............. & 223707.6 & +342249 & 0.62 & 12 & 39.97 & 39.76 & 39.56 & 38.86 & 40.03 \\
\hline UV+IR-74 ................ & 223706.5 & +342724 & 0.60 & 20 & 39.27 & 39.12 & 39.71 & 38.97 & 40.16 \\
\hline UV+IR-75 ............... & 223710.0 & +342239 & 0.51 & 19 & 39.67 & 39.70 & 39.76 & 38.85 & 40.12 \\
\hline UV+IR-76 ............... & 223654.4 & +342629 & 0.53 & 33 & 40.02 & 39.85 & 39.92 & 38.94 & 40.24 \\
\hline UV+IR-77 .................. & 223700.8 & +342447 & 0.39 & 13 & 39.93 & 39.91 & 39.74 & 39.09 & 40.24 \\
\hline UV+IR-78 ............... & 223659.7 & +343015 & 0.32 & 23 & 39.31 & 39.02 & 39.47 & 38.69 & 39.89 \\
\hline UV+IR-79 ............... & 223703.6 & +342848 & 0.29 & 20 & 39.26 & 39.32 & 39.20 & 38.54 & 39.69 \\
\hline UV+IR-80 ............... & 223651.1 & +343227 & 0.29 & 42 & 39.32 & 39.38 & 39.29 & 38.41 & 39.67 \\
\hline UV+IR-81 ............... & 223705.9 & +342857 & 0.31 & 26 & 40.14 & 40.12 & 39.41 & 38.35 & 39.69 \\
\hline UV+IR-82 ............... & 223715.1 & +341835 & 0.35 & 36 & 40.00 & 39.82 & 39.46 & 38.67 & 39.88 \\
\hline UV+IR-83 ............... & 223658.5 & +342751 & 0.61 & 18 & 39.83 & 39.74 & 39.40 & 38.76 & 39.90 \\
\hline UV+IR-84 ............... & 223652.5 & +343142 & 0.36 & 38 & 39.42 & 39.48 & 39.47 & 39.52 & 40.33 \\
\hline UV+IR-85 ................ & 223655.3 & +343136 & 0.38 & 32 & 39.57 & 39.66 & 39.07 & 38.94 & 39.84 \\
\hline 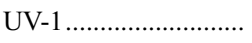 & 223704.0 & +342348 & 0.94 & 6 & 41.00 & 40.99 & 40.37 & 39.72 & 40.86 \\
\hline 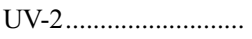 & 223706.7 & +342620 & 0.63 & 16 & 40.76 & 40.71 & 40.82 & 40.28 & 41.37 \\
\hline UV-3 ....................... & 223706.4 & +342056 & 0.79 & 18 & 40.53 & 40.47 & 40.04 & 39.25 & 40.46 \\
\hline 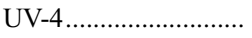 & 223705.0 & +342449 & 0.37 & 3 & 40.59 & 40.89 & 41.30 & 40.52 & 41.73 \\
\hline 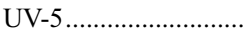 & 223701.7 & +342853 & 0.48 & 17 & 40.45 & 40.32 & 40.32 & 39.71 & 40.83 \\
\hline 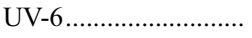 & 223707.0 & +342114 & 0.43 & 16 & 40.47 & 40.32 & 40.28 & 39.53 & 40.72 \\
\hline 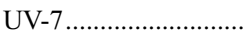 & 223702.2 & +342405 & 0.51 & 11 & 40.58 & 40.42 & 40.80 & 40.27 & 41.35 \\
\hline 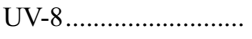 & 223701.6 & +342518 & 0.40 & 9 & 40.55 & 40.54 & 40.19 & 39.81 & 40.82 \\
\hline 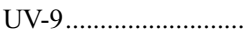 & 223710.2 & +342202 & 0.71 & 19 & 40.65 & 40.52 & 40.44 & 39.80 & 40.94 \\
\hline 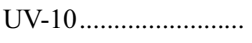 & 223704.8 & +342500 & 0.33 & 3 & 40.47 & 40.72 & 41.24 & 40.48 & 41.67 \\
\hline 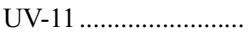 & 223706.6 & +342156 & 0.52 & 13 & 40.45 & 40.28 & 39.47 & 38.76 & 39.93 \\
\hline 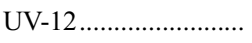 & 223701.2 & +342612 & 0.40 & 9 & 40.26 & 40.31 & 40.73 & 40.03 & 41.20 \\
\hline 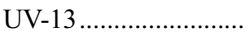 & 223707.1 & +342610 & 0.55 & 16 & 40.52 & 40.37 & 39.73 & 38.88 & 40.12 \\
\hline UV-14 „........................ & 223704.4 & +342515 & 0.67 & 3 & 40.59 & 40.88 & 41.39 & 40.61 & 41.82 \\
\hline UV-15 ......................... & 223703.5 & +342329 & 0.59 & 9 & 40.34 & 40.27 & 40.65 & 39.95 & 41.12 \\
\hline 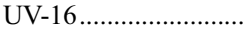 & 223705.6 & +342254 & 0.49 & 9 & 40.25 & 40.14 & 39.98 & 39.20 & 40.41 \\
\hline 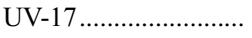 & 223702.8 & +342345 & 0.47 & 10 & 40.18 & 39.99 & 39.63 & 38.82 & 40.04 \\
\hline 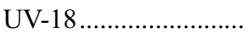 & 223707.6 & +342250 & 0.60 & 11 & 40.45 & 40.33 & 39.96 & 39.03 & 40.30 \\
\hline 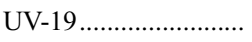 & 223704.5 & +342109 & 1.09 & 19 & 40.47 & 40.27 & 40.48 & 39.69 & 40.90 \\
\hline 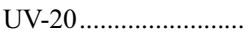 & 223707.0 & +342454 & 0.57 & 11 & 40.23 & 40.19 & 40.54 & 40.30 & 41.25 \\
\hline UV-21 ......................... & 223710.1 & +342046 & 0.53 & 21 & 40.32 & 40.13 & 40.06 & 39.33 & 40.51 \\
\hline 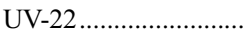 & 223705.9 & +342754 & 0.61 & 21 & 40.32 & 40.29 & 39.58 & 38.57 & 39.89 \\
\hline 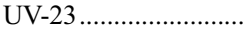 & 223701.8 & +342642 & 0.44 & 8 & 40.17 & 40.13 & 40.54 & 39.78 & 40.98 \\
\hline UV-24 ......................... & 223708.8 & +342033 & 0.79 & 20 & 40.42 & 40.37 & 40.21 & 39.47 & 40.66 \\
\hline UV-25 ........................ & 223700.1 & +342907 & 0.69 & 18 & 40.39 & 40.21 & 39.81 & 38.96 & 40.20 \\
\hline 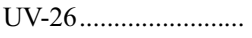 & 223659.7 & +342639 & 0.44 & 14 & 39.90 & 39.82 & 39.91 & 39.43 & 40.49 \\
\hline UV-27 ......................... & 223658.7 & +342621 & 0.33 & 17 & 39.94 & 39.80 & 39.46 & 38.71 & 39.90 \\
\hline 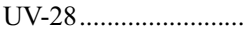 & 223707.6 & +342622 & 0.32 & 19 & 40.01 & 39.76 & 38.45 & 38.51 & 39.31 \\
\hline 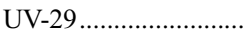 & 223704.7 & +342251 & 0.52 & 10 & 40.48 & 40.32 & 39.66 & 38.87 & 40.08 \\
\hline 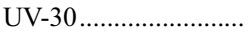 & 223710.4 & +342101 & 0.31 & 21 & 39.99 & 39.82 & 39.90 & 39.18 & 40.36 \\
\hline UV-31 ........................ & 223659.5 & +342837 & 0.81 & 17 & 40.19 & 40.04 & 39.76 & 38.83 & 40.11 \\
\hline 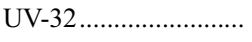 & 223701.6 & +342845 & 0.42 & 17 & 40.12 & 40.06 & 39.42 & 38.85 & 39.95 \\
\hline UV-33 „....................... & 223708.1 & +342142 & 0.30 & 15 & 39.86 & 39.74 & 40.07 & 39.40 & 40.55 \\
\hline 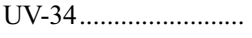 & 223705.1 & +342629 & 0.75 & 11 & 40.21 & 40.16 & 39.67 & 39.01 & 40.16 \\
\hline 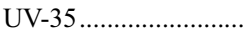 & 223710.4 & +342148 & 0.32 & 20 & 40.07 & 39.94 & 40.06 & 39.31 & 40.50 \\
\hline UV-36 ....................... & 223705.4 & +342148 & 0.31 & 14 & 39.85 & 39.67 & 39.67 & 38.76 & 40.03 \\
\hline UV-37 ......................... & 223703.8 & +342529 & 0.44 & 2 & 40.09 & 40.56 & 41.13 & 40.64 & 41.71 \\
\hline 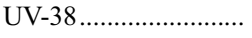 & 223705.0 & +342330 & 0.35 & 6 & 39.90 & 39.91 & 40.28 & 39.51 & 40.71 \\
\hline 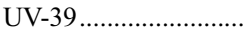 & 223659.2 & +342742 & 0.37 & 16 & 40.03 & 39.82 & 39.76 & 39.03 & 40.21 \\
\hline UV-40 ........................ & 223704.8 & +342531 & 0.36 & 5 & 39.92 & 40.08 & 41.17 & 40.64 & 41.73 \\
\hline 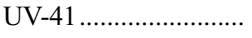 & 223707.4 & +342644 & 0.41 & 20 & 40.06 & 39.99 & 39.65 & 38.85 & 40.07 \\
\hline 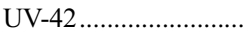 & 223705.6 & +342456 & 0.31 & 6 & 40.26 & 40.55 & 41.37 & 40.84 & 41.92 \\
\hline UV-43 ....................... & 223707.5 & +342310 & 0.31 & 11 & 39.95 & 39.78 & 40.16 & 39.47 & 40.63 \\
\hline 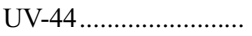 & 223707.3 & +342211 & 0.43 & 13 & 40.01 & 39.86 & 39.88 & 38.97 & 40.24 \\
\hline
\end{tabular}


TABLE 2-Continued

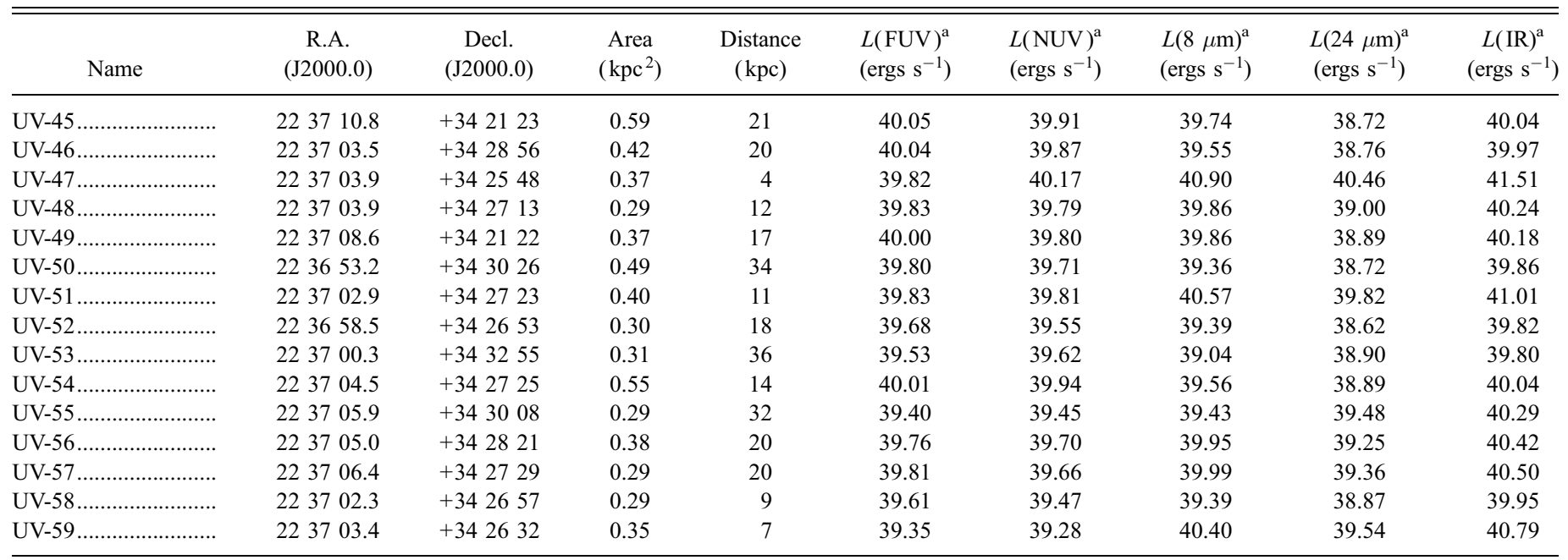

NoтE.- -Units of right ascension are hours, minutes, and seconds, and units of declination are degrees, arcminutes, and arcseconds.

a The maximum $1 \sigma$ uncertainty in reported luminosity values for sources included in this table is 0.09 dex.

also used for boundary definition. Full discussion of deblendingrelated parameters is deferred to D. Thilker et al. (2008, in preparation), but they were set so as to break apart individual peaks inside larger composite objects. Integrated flux measurements within these irregularly shaped apertures were extracted from each of the bands regardless of the selection bandpass. The background level for each substructure was determined after the extent of all regions from both selection images was finalized. These areas were masked, and we robustly determined the mode of the remaining unmasked pixels in a circular region around each source. Table 2 presents our flux measurements for all significant substructures in the UV+IR- and UV-selected samples. Analysis of our photometry is discussed in $\S 4.4$.

To accomplish the later goal, we constructed a datacube containing the flux-calibrated images at all available wavelengths. During the cube-making process, each image was registered to a master coordinate grid after convolution to a common resolution. This data product could then be used directly to assess the SED shape at any given position, such as the brightest peaks of the dust ring or, conversely, in quiescent locales. A series of such cubes were generated that were matched to the limiting angular resolution of the MIPS 24, 70, and $160 \mu \mathrm{m}$ imagery.

To examine the dependence of SED shape on galactocentric radius and thereby determine the variation of SFR, star formation history (SFH), and dust properties in a galaxy-wide context, we computed radial surface brightness profiles evaluated within concentric elliptical annuli. The adopted inclination, $i=77^{\circ}$, and position angle, $170^{\circ}$, were taken from García-Gómez et al. (2002). Due to the varied resolution of our multispectral data set, we evaluated radial profiles at differing angular resolution using the flux-calibrated datacubes described above. The image data were blanked of foreground stars prior to generating the galaxy profiles. The highest resolution considered was $6^{\prime \prime}$, permitting inclusion of the GALEX, optical, Two Micron All Sky Survey (2MASS), IRAC, and MIPS $24 \mu \mathrm{m}$ imagery. These profiles (shown in Fig. 4) allow us to constrain the emergent stellar SED and the properties of warm dust but contain very little information on the complete SED of dust grains. To gain more direct measurements regarding the long-wavelength MIPS bands, we computed lower resolution profiles (see Fig. 4) using the cubes convolved to $\left(18^{\prime \prime}\right) 40^{\prime \prime}[1.3(2.8) \mathrm{kpc}] \mathrm{FWHM}$, thereby matching the 70 (160) $\mu \mathrm{m}$ PSF. We integrated the radial profiles to obtain total flux

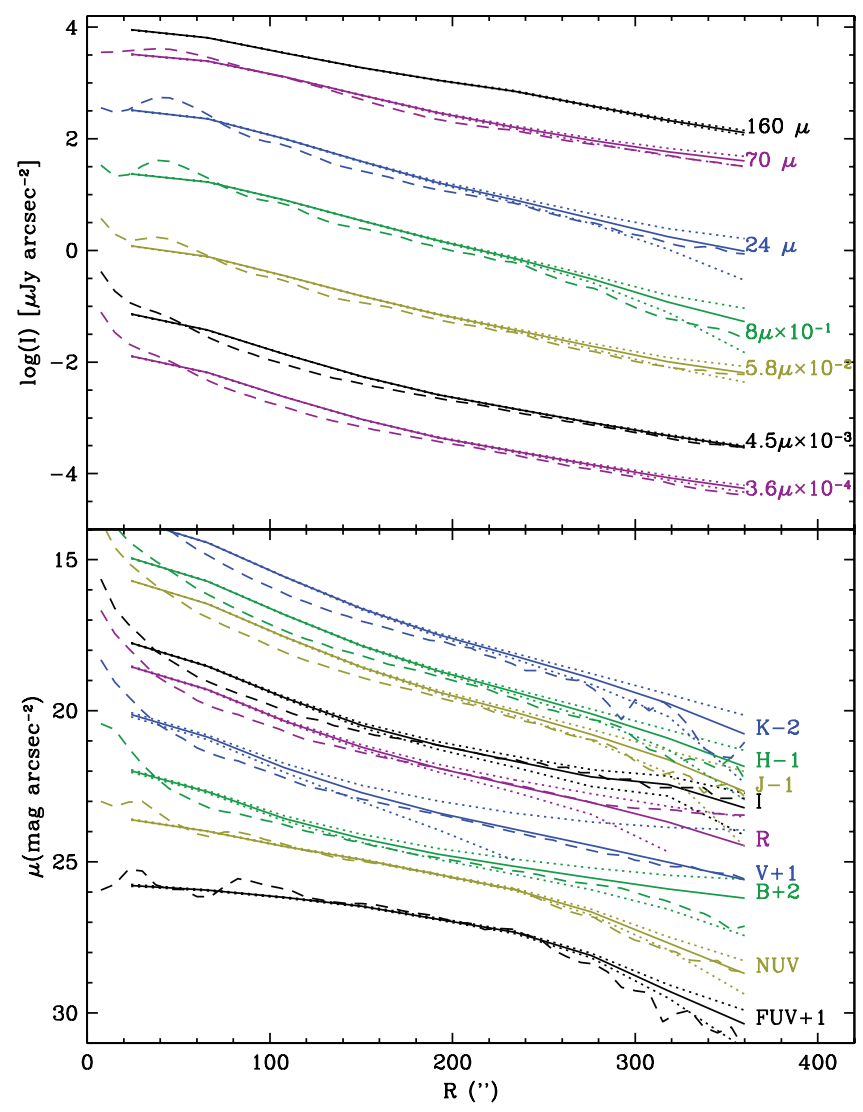

FIG. 4.- Radial surface brightness profiles as a function of galactocentric radius in NGC 7331. We include all the Spitzer bands in the top panel. The bottom panel shows measurements from 2MASS (NIR: $J H K$ ), visible (BVRI), and GALEX (NUV and FUV) bands. The solid lines indicate the profiles measured at resolution corresponding to the limit of our $160 \mu \mathrm{m}$ imagery, whereas the dashed lines show higher (intrinsic) resolution profiles. The dotted lines represent the $1 \sigma$ uncertainty of the former profiles and are clearly a lower limit to the uncertainty of the highresolution measurements. Note that the star-forming ring is distinguishable in the high-resolution profiles. 
TABLE 3

Flux Measurements and SFR Estimates for NGC 7331

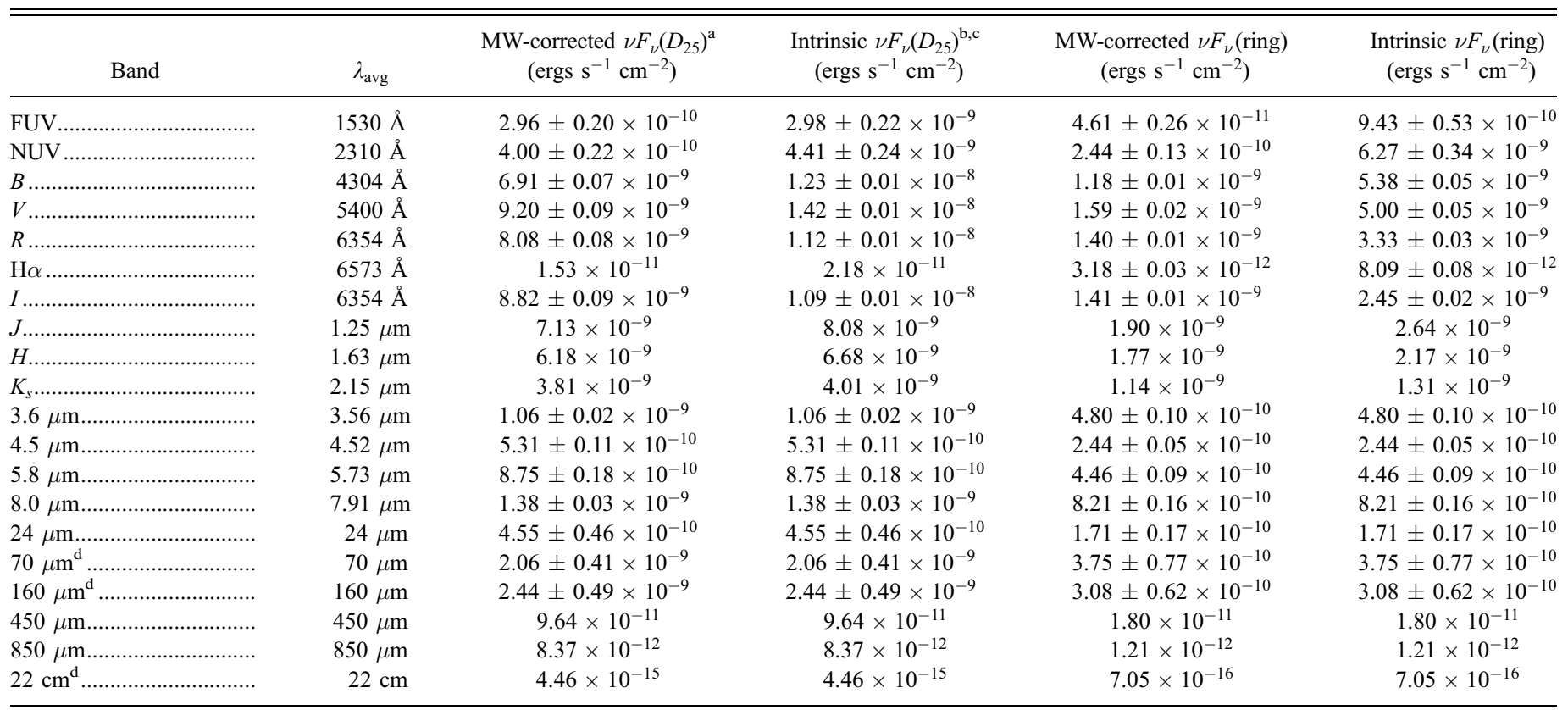

${ }^{\text {a }}$ Following DH02 and using our 24-160 $\mu \mathrm{m}$ flux measurements, we estimate global total IR $=5.58 \times 10^{-9} \mathrm{ergs} \mathrm{s}^{-1} \mathrm{~cm}^{-2}$. The ring's total IR flux was estimated by scaling our $24 \mu \mathrm{m}$ measurement according to eq. (1), as the resolution of the Spitzer 70 and $160 \mu \mathrm{m}$ images is low compared to the dimensions of the ring. We obtain total $\mathrm{IR}=2.02 \times 10^{-9} \mathrm{ergs} \mathrm{s}^{-1} \mathrm{~cm}^{-2}$ for the ring.

${ }^{\mathrm{b}}$ Based on the parameterization of Burgarella et al. (2005), we obtain global $A(\mathrm{FUV})=2.51$ and ring $A(\mathrm{FUV})=3.3$. The intrinsic SED values presented in this table were computed following dust correction to this degree using a Milky Way extinction law. The reddest band corrected for extinction was $K_{s}$; at longer wavelengths the extinction is negligible.

${ }^{\circ}$ SFR estimates in solar masses per year: global (FUV: 6.3, H $\alpha: 4.4,22 \mathrm{~cm}: 5.1$, IR: 4.6, bol: 4.9) and ring (FUV: 1.9, H $\alpha: 1.6,22 \mathrm{cm:} 0.8$, IR: 1.6, bol: 1.7)

${ }^{d}$ In these bands the measured fluxes are underestimates given the comparitively low resolution of the data relative to NGC 7331's ring.

measurements for NGC 7331 within the $D_{25}$ extent of the galaxy. The global SED will be discussed in $\S 4.1$ and the radial profiles in $\S 4.3$.

\section{RESULTS AND DISCUSSION}

\subsection{Global Spectral Energy Distribution, Star Formation Rate, and Stellar Mass}

Table 3 presents our integrated flux measurements within $D_{25}$ for NGC 7331. We list the data simply corrected for Galactic foreground extinction $[E(B-V)=0.091 \mathrm{mag}]$ and in a form additionally dereddened on the basis of an internal extinction estimate. The global attenuation at FUV was determined using the formulation of Burgarella et al. (2005) and Buat et al. (2005), which parameterizes $A(\mathrm{FUV})$ as a function of $L(\mathrm{IR}) / L(\mathrm{UV})$ and is relatively insensitive to SFH (see Appendix B). With the measurements in Table 3 , we derive $A_{\text {global }}($ FUV) $\sim 2.51$ mag. For the global dereddening in other bands, we further assumed a Milky Way attenuation law given the apparent similarity between NGC 7331 and the Galaxy. Buat et al. (2005) show that the dispersion in the $L(\mathrm{IR}) / L$ (UV)- $A$ (UV) calibration caused by use of different attenuation laws is only about $5 \%$, so our choice of the Milky Way law for bands other than FUV should yield a consistently dereddened SED.

Applying the formulae of $\S 3.3$ and Appendix B to the dustcorrected FUV and $\mathrm{H} \alpha$ luminosities and the extinction-free total IR and radio measurements, we obtained estimates of the total SFR in NGC 7331, which are given in a footnote to Table 3. Within the photometric errors and considering the different timescales being probed, the FUV, $\mathrm{H} \alpha$, radio continuum, IR, and bolometric SFRs are in agreement, spanning the range 4.4-6.3 $M_{\odot} \mathrm{yr}^{-1}$.

Comparing the $2 \mathrm{MASS}$ total $K_{S}$ magnitude with populationsynthesis models for continuous star formation, solar metallicity, and a Chabrier initial mass function (IMF) (Bruzual \& Charlot 2003; Chabrier 2003), we obtain an estimate of the total stellar mass in NGC 7331 amounting to $5.3 \times 10^{10} M_{\odot}$. This is somewhat smaller than the recent determination $\left(1.3 \times 10^{11} M_{\odot}\right)$ of McGaugh (2005), probably owing to differences in the assumed mass-to-light ratio $(M / L)$. This ratio is uncertain even for a single galaxy, as it depends on the detailed SFH of the object. (The range in $M / L$ considered by McGaugh spans a factor of 4.) Assuming their population synthesis-based $M / L$ reduces their mass estimate to $7.4 \times 10^{10} M_{\odot}$, which is in rough agreement with our analysis of the 2MASS data. In any case, the total stellar mass of NGC 7331 is 3-4 times the gaseous mass, including both $\mathrm{H}$ I (Braun et al. 2003) and molecular (Regan et al. 2004) components. This ratio is common for galaxies with a stellar mass $\gtrsim 10^{10} M_{\odot}$, although a tail in the distribution reaches to substantially greater ratios.

\subsection{Star Formation within NGC 7331 's Dust Ring}

How significant is the highly obscured star formation now occurring in NGC 7331's dust ring? In addition to the global SED, Table 3 also presents observed and extinction-corrected FUVradio flux measurements for the ring. Note that, in obtaining these measurements, we have subtracted an underlying contribution from the disk by evaluating a local, average background level outside of the dust ring. Our multiwavelength SFR estimates for the ring are in agreement $\left(1.6-1.9 M_{\odot} \mathrm{yr}^{-1}\right)$, with the exception of the radio continuum value $\left(0.8 M_{\odot} \mathrm{yr}^{-1}\right)$. However, the $22.6^{\prime \prime} \times$ $11.0^{\prime \prime}$ resolution of the WSRT map certainly led to some of the ring flux falling outside of our image mask used to define the ring at other wavelengths. Note that the total IR flux was computed by scaling the $24 \mu \mathrm{m}$ measurement according to equation (1). In any case, the ring contributes approximately one-third of the present star-formation activity in NGC 7331. 
From the comparison of UV and IR morphology in $\S 3.1$, it is obvious that the star-forming ring is highly extincted at UV wavelengths. The UV extinction determined specifically from the integrated ring photometry via the Burgarella et al. (2005) $L(\mathrm{IR}) / L(\mathrm{UV})$ formulation is $A_{\text {ring }}(\mathrm{FUV})=3.3$.

The estimated SFR can be coupled with the gas mass of the ring to determine a gas-consumption timescale, assuming no gas recycling and/or fueling via radial gas motions. Regan et al. (2004) estimated the total gas mass of the ring to be $4 \times 10^{9} M_{\odot}$, comprised of $3.4 \times 10^{9} M_{\odot}$ in molecular form and $5.6 \times 10^{8} M_{\odot}$, in atomic form. Throughout our analysis we have assumed a metallicity-dependent $X$-factor to convert from $\mathrm{CO}$ integrated intensity to $N\left(\mathrm{H}_{2}\right)$. Dutil \& Roy (1999) show that the oxygen abundance of the ring environment is consistent with $12+\log [\mathrm{O} / \mathrm{H}] \sim$ 9.1. Using the $X$-factor calibration of Boselli et al. (2002), we adopted a $\mathrm{CO}$ conversion factor of $1.2 \times 10^{20} \mathrm{~cm}^{-2}\left(\mathrm{~K} \mathrm{~km} \mathrm{~s}^{-1}\right)^{-1}$. This change reduces the Regan et al. measurement for total gas mass in the ring to $2 \times 10^{9} M_{\odot}$. The corresponding gas-consumption timescale is $>1$ Gyr. Evidently, the dusty star-forming ring of NGC 7331 has the potential to remain a long-standing morphological feature fueled only by the ISM reservoir already amassed, unless otherwise quenched by dynamical means. Our UV and IR data show that there is very little (if any) current star formation interior to the ring, which is consistent with the absence of $\mathrm{CO}$ and $\mathrm{H} \mathrm{I}$ in the circumnuclear regions $\left(d \lesssim 2 \mathrm{kpc}, 28^{\prime \prime}\right)$ of NGC 7331 (see Fig. 1).

\subsection{Radial Variation of Star Formation History, Extinction, and Dust Temperature}

Figure 4 shows low- and moderate-resolution radial surface brightness profiles for NGC 7331, the computation of which was described in $\S 3.4$. The low-resolution profiles (solid lines) were measured at resolution corresponding to the limit of our $160 \mu \mathrm{m}$ imagery.

The star-forming ring and stellar bulge become distinguishable in higher resolution profiles shown as dashed lines in Figure 4. The ring is a prominent dust emission feature showing notable enhancement at galactocentric distances of $20-80^{\prime \prime}(1.4-5.7 \mathrm{kpc})$ over wavelengths ranging from 70 to $5.8 \mu \mathrm{m}$. The SCUBA imagery of Figure 1 demonstrates that the ring morphology persists even to $850 \mu \mathrm{m}$, despite not being resolved by our Spitzer $160 \mu \mathrm{m}$ data. In the high-resolution profiles (dashed lines), the star-forming ring appears to be noticeable as a very slight, local flattening away from otherwise smoothly declining radial profiles in the 4.5 and $3.6 \mu \mathrm{m}$ bands. This could indicate that the star-forming ring is not newly formed and has produced a significant perturbation within the mass surface density of the stellar disk. A second explanation may be that the ring is a dynamical structure including stars and gas. Deciding between these possibilities requires detailed analysis of the kinematic information contained in the $\mathrm{H}$ I and $\mathrm{CO}$ datacubes, but such a study is beyond the scope of this paper. Alternatively, note that Regan et al. demonstrate that a small fraction $(6 \%)$ of the $4.5 \mu \mathrm{m}$ emission originates from very hot $(1000 \mathrm{~K})$ dust grains. Therefore, hot dust emission may partially contribute to the inflection at $4.5 \mu \mathrm{m}$ but probably not at $3.6 \mu \mathrm{m}$. Note that the presence of red supergiants in the star-forming regions of the ring could also potentially reduce the effective $M / L$ for the 3.6 and $4.5 \mu \mathrm{m}$ IRAC bands.

The FUV-NUV color becomes slightly bluer $(\sim 0.5 \mathrm{mag})$ with increasing radius when measured at $160 \mu \mathrm{m}$ resolution, and FUV $-K$ becomes bluer by about $3 \mathrm{mag}$ from the galaxy center to the outer edge. We show below that this is fully consistent with a change in SFH as a function of galactocentric distance, as quantified by the $100 \mathrm{Myr}$ birthrate parameter, $b_{8}$. We define $b_{8}$ as the

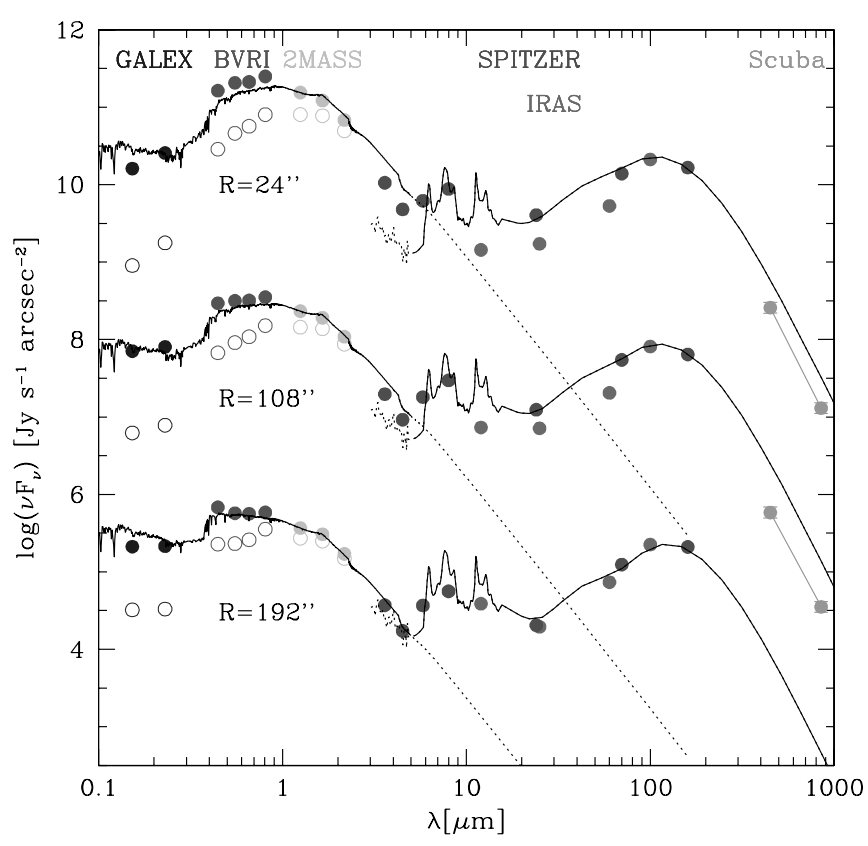

FIG. 5.- Average 0.15-1000 $\mu \mathrm{m}$ SEDs at three different radii. In the UV-NIR range, open symbols show observed values, and filled symbols indicate extinctioncorrected values (see text). The SEDs were computed in successive radial bins sized in accordance with the Spitzer $160 \mu \mathrm{m}$ PSF FWHM of $\sim 40^{\prime \prime}$. Only every other bin is shown here, each being offset for clarity by 2 dex downward from the previous one. The data in each band were convolved to the $160 \mu \mathrm{m}$ resolution after star removal and before profile measurement. The curves (on the IR side) are a fit of the Spitzer points by a DH02 model (details in text). The SCUBA points were not included in the fit as the observations cover only part of the galaxy; they, however, are close to the predictions for the fit performed at lower wavelengths, and the observed slope is in agreement with the model one. The UV-optical-NIR dust-corrected SED is fitted with a simple population-synthesis model corresponding to an exponentially decreasing SFR history. The model fits suggest about an order-of-magnitude increase in $b_{8}$ from the inner $1.5^{\prime}$ to the outer edge of the disk. Over the same range, the heating intensity distribution must be notably "cooler" to remain consistent with the observed IR SED. [See the electronic edition of the Supplement for a color version of this figure.]

ratio of the SFR averaged over the last $100 \mathrm{Myr}$ to the SFR averaged over the Hubble time. Note the difference with regard to $b_{0}$, the instantaneous birthrate parameter. Muñoz-Mateos et al. (2007) show a systematic increase in $b_{8}$ as a function of galactocentric distance for a larger sample of galaxies observed by GALEX and 2MASS.

Figure 5 presents the average $0.15-1000 \mu \mathrm{m}$ SED as a function of galactocentric distance, obtained from the lower resolution profiles of Figure 4 (only a few annuli are shown). Overplotted are models of the dust emission and UV-NIR starlight (from the aggregate stellar population) in each radial bin. The best fit was determined as follows. We initially compared the Spitzer fluxes to the parameterized dust models of DH02. These authors presented their models as a function of the power-law index, $\alpha$, of the heating-intensity distribution function. Higher values of $\alpha$ correspond to environments preferentially lacking the relatively hard interstellar radiation field. Our IR observations are best described by values of $\alpha$ ranging from 2.19 near the center of NGC 7331 up to 2.94 in the radial bin centered at $234^{\prime \prime}(17 \mathrm{kpc})$. See Table 4 for measurements and fitting results. That is, the apparent dust heating-intensity distribution becomes "cooler" with increasing galactocentric distance, possibly reflecting a change in the relative strength of dust-heating mechanisms (e.g., arm vs. interarm environments dominated by young and old stars, respectively). Having fit the dust emission in this manner, we can compute the total FIR emission by integrating the model spectrum (equivalent 
TABLE 4

Results from SED Fitting within Annular Zones

\begin{tabular}{|c|c|c|c|c|c|c|c|c|}
\hline \multicolumn{2}{|l|}{ Radius } & $\alpha_{\mathrm{DH} 02}$ & $\begin{array}{c}\log F(\text { dust }) \\
\left(\mathrm{W} \mathrm{m}^{-2} \operatorname{arcsec}^{-2}\right)\end{array}$ & $\begin{array}{l}\log \nu F_{\nu, \mathrm{obs}}(\mathrm{FUV}) \\
\left(\mathrm{W} \mathrm{m} \mathrm{m}^{-2} \operatorname{arcsec}^{-2}\right)\end{array}$ & $\log \left[F(\right.$ dust $) / \nu F_{\nu, \text { obs }}($ FUV $\left.)\right]$ & $\begin{array}{l}A(\mathrm{FUV}) \\
(\mathrm{mag})\end{array}$ & $\begin{array}{c}\log \Sigma_{\mathrm{SFR}} \\
\left(M_{\odot} \mathrm{pc}^{-2} \mathrm{Gyr}^{-1}\right)\end{array}$ & $b_{8}$ \\
\hline $3-45 \ldots$ & $0.2-3.2$ & 2.19 & -15.4 & -17.0 & 1.7 & 3.3 & 2.2 & 0.028 \\
\hline $45-87 \ldots \ldots \ldots \ldots \ldots \ldots \ldots . .$. & $3.2-6.2$ & 2.19 & -15.5 & -17.1 & 1.6 & 3.2 & 2.0 & 0.035 \\
\hline $87-129 \ldots \ldots \ldots \ldots \ldots \ldots$ & $6.2-9.2$ & 2.31 & -15.8 & -17.2 & 1.4 & 2.8 & 1.8 & 0.059 \\
\hline $129-171 \ldots \ldots \ldots \ldots \ldots . .$. & $9.2-12.2$ & 2.50 & -16.1 & -17.3 & 1.2 & 2.4 & 1.5 & 0.059 \\
\hline $171-213 \ldots \ldots \ldots \ldots \ldots \ldots$ & $12.2-15.2$ & 2.81 & -16.4 & -17.5 & 1.1 & 2.2 & 1.2 & 0.214 \\
\hline $297-339 \ldots \ldots \ldots \ldots \ldots . .$. & $21.2-24.2$ & 2.75 & -17.1 & -18.4 & 1.3 & 2.7 & 0.50 & 0.186 \\
\hline
\end{tabular}

to using eq. [4] of DH02) and use the FIR/FUV ratio to deduce the degree of extinction at UV wavelengths in each radial bin (using the Burgarella et al. [2005] calibration). The extinction at other wavelengths up to the NIR are then estimated using the simplistic model of Boselli et al. (2003), which was also used in Boissier et al. (2004).

Figure 5 shows the observed values (open circles) and those corrected for the estimated extinction as described above ( filled circles). The extinction correction is extremely large, especially in the UV and should be considered as quite uncertain. This uncertainty in the extinction-corrected SEDs propagates throughout our profile analysis, especially since aging and extinction produce similar reddening effects.

Nevertheless, to obtain a rough idea of the radial variation of the azimuthally averaged SFH in NGC 7331, a suite of populationsynthesis models (using the codes of Boissier \& Prantzos 1999) having SFHs given by exponentials with different timescales is compared with the dereddened colors relative to $K$. These synthesis models are further constrained to remain in broad agreement with the nebular abundance of oxygen (Dutil \& Roy 1999). We find that the best-fit SFH varies in the sense that values of $b_{8}$ increase by almost an order of magnitude from the galaxy center to the $D_{25}$ radius ( $b_{8}=0.028$ for the $24^{\prime \prime}[1.7 \mathrm{kpc}]$ SED in Figure 5 vs. $b_{8}=0.214$ at $276^{\prime \prime}$, or $20 \mathrm{kpc}$ ). The spectrum corresponding to the best-fit SFH for each radial bin is included in Figure 5.

It has been relatively common to fit the FIR SED of galaxies as a double-modified ( $\lambda^{-2}$ emissivity) blackbody to gain some knowledge of the properties (e.g., temperature, mass) of the dust they contain (e.g., Popescu et al. 2002; Boselli et al. 2003).

As an alternative to the DH02 fit, enabling comparison with the literature, we also performed a fit of the MIPS fluxes and IRAS $100 \mu \mathrm{m}$ data by a double-modified black-body SED to determine warm and cold dust temperatures. Adding the $100 \mu \mathrm{m}$ to the MIPS data gives us as many constraints as parameters. The IRAS data were obtained by a HIRES request (from IRSA). The $100 \mu \mathrm{m}$ image has a PSF broader (by a factor of $>2$ ) than that we used (matching $160 \mu \mathrm{m}$ ), but we confirmed that we obtain similar results after degrading all the data to the $100 \mu \mathrm{m}$ resolution. That is, all structures have already been smoothed out; thus, the difference in resolution has a weak impact at this point. We note that at the shortest wavelength, the IRAS images are of very poor quality (the PSF is very asymmetric and orthogonal to the position angle of the galaxy), which might explain the disagreement between the Spitzer and IRAS points.

The results of this double-modified black-body fit is presented in Figure 6 . The temperature $T_{\text {cold }}$ (dotted line) has a nearly constant value of $20 \mathrm{~K}$ as can be seen in Figure $6 a$. The value of $T_{\text {warm }}$ (dashed line) is less well constrained, as warm dust only dominates the $24 \mu \mathrm{m}$ band. Still, $T_{\text {warm }}$ shows signs of a decrease with radius, in agreement with our analysis of Figure 5. Figure $6 b$ plots integrated IR flux density associated with the warm and cold components as a function of radius. The solid line shows the combined (warm and cold) flux density, which is found to be comparable to the total IR radiation obtained by fitting the dust SED with the DH02 models (magenta). The ratio of $F_{\text {dust }}$ to $F_{\mathrm{UV}}$ for both GALEX bands (NUV, dashed lines; FUV, solid lines) is shown in Figure $6 c$. The origin of the deviation to larger ratios at radii near $R_{25}$ (largest radius where it was computed) could be explained by one of the two phenomena below (or a combination of them). (1) The galaxy presents prominent spiral arms, including close to $R_{25}$. The increase in extinction could be due to the fact that this particular radial bin includes a lot of extincted starforming regions (the bin just inside that one could be associated with regions of lower extinction in interarm areas). (2) In the outer low-density regions, some of the dust might be located far from bright star-forming regions and might be partially heated by relatively distant older stars. In that case, the balance of FUV and FIR radiation would not be representative of the local UV extinction, and we would overestimate the local extinction with our methodology.

Figure $6 d$ shows the implied extinction as a function of galactocentric distance, determined using the relation of Burgarella et al. (2005). The azimuthally averaged values presented are in good agreement with the estimates presented later in the paper for individual sources, in the sense that the averaged values are lower or equivalent to discrete measures at fixed radii (with the caveat already noted for the outer annulus).

This global decrease of extinction with radius (except for the last point, as discussed above) is in agreement with a general trend observed in six late-type galaxies by Boissier et al. (2004) based on IRAS and FOCA data, in M101 by Popescu et al. (2005) based on GALEX and ISO data, and in Holwerda et al. (2004, 2005a, $2005 \mathrm{~b}$ ) based on the independent method of background-galaxies counts. See also Boissier et al. (2007) for a comprehensive look at the issue using GALEX and IRAS observations.

In Boissier et al. (2004), it was suggested that the decrease in extinction is associated with the metallicity gradient usually observed in spiral galaxies. In NGC 7331, Dutil \& Roy (1999) used narrowband imaging to measure the nebular oxygen abundance in $164 \mathrm{H}$ II regions. From these data they determined a metallicity gradient of the form $Z=12+\log (\mathrm{O} / \mathrm{H})=(9.15 \pm 0.014)-$ $(0.021 \pm 0.0015) \mathrm{dex} \mathrm{kpc}^{-1}$. Adopting the extinction-metallicity relation of Boissier et al. (2004) with this gradient, we would obtain a predicted variation in extinction of $\sim 1.3$ mag over the radial range studied. The observed one is a bit smaller, but the UV wavelengths probed in the two studies differ (FOCA vs. GALEX), the dispersion between galaxies in Boissier et al. (2004) is large, and NGC 7331 is more inclined than the galaxies in that study. Calzetti et al. (1994) 
(a)

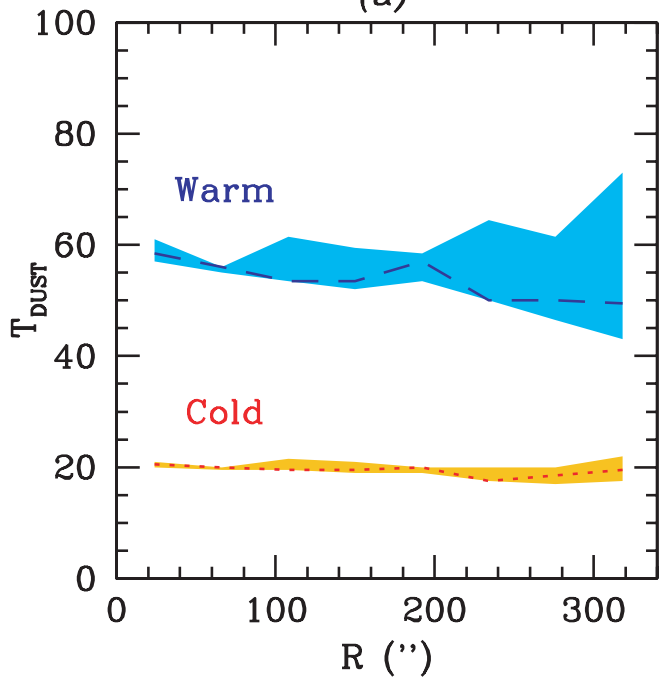

(b)

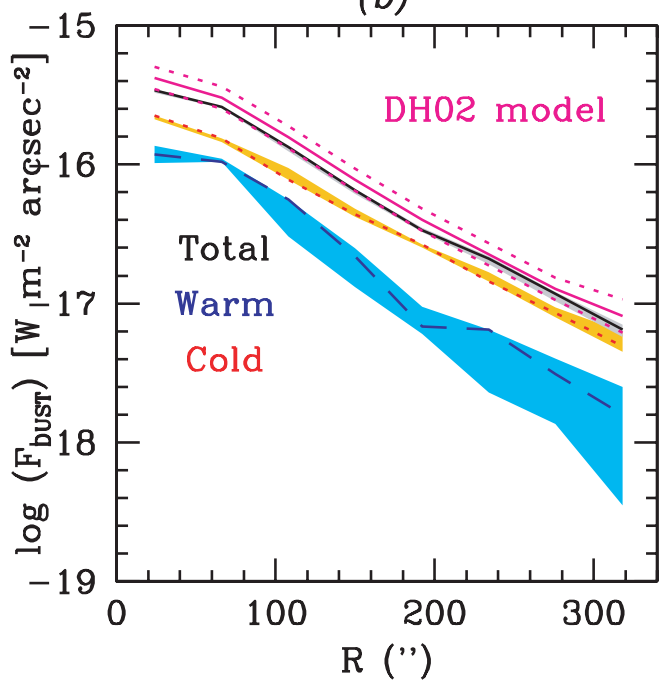

(c)

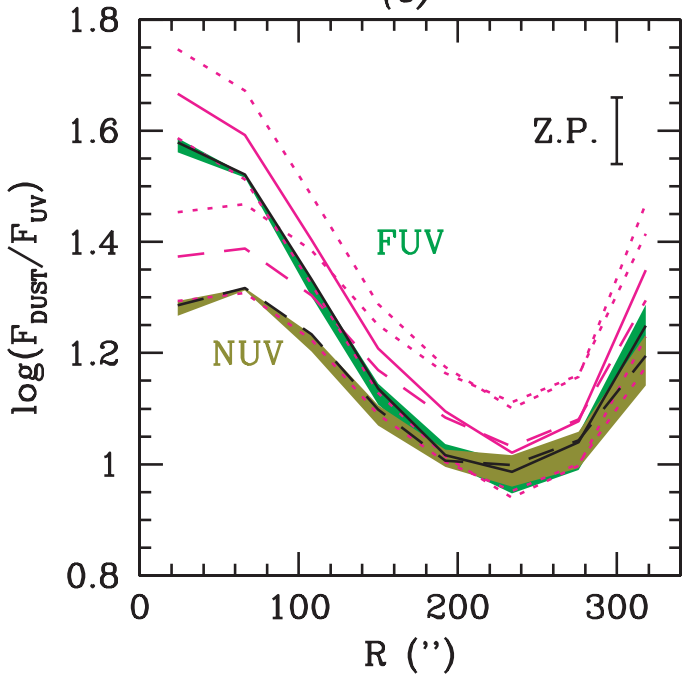

(d)

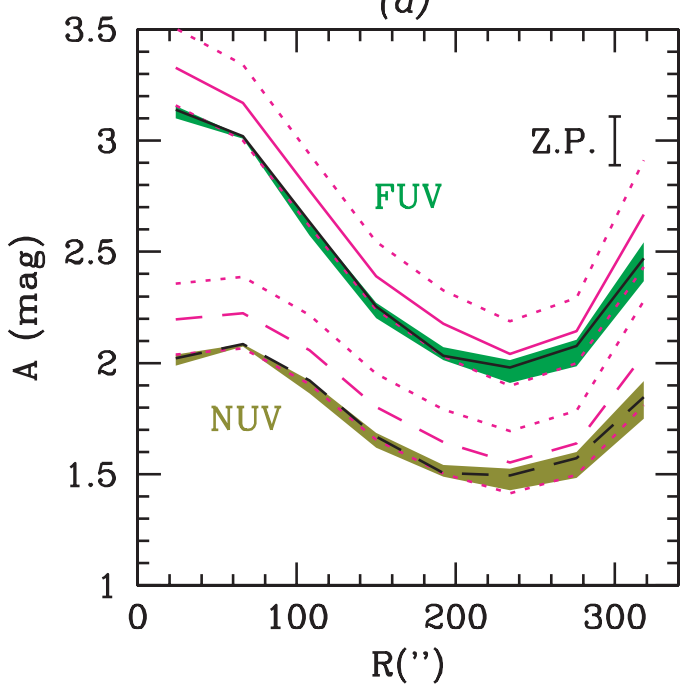

FIG. 6. - Radial variation of the dust properties and extinction in NGC 7331. (a) Dust temperatures determined by fitting a double-modified blackbody SED to the MIPS bands

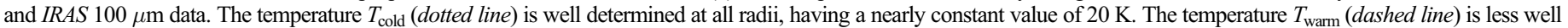

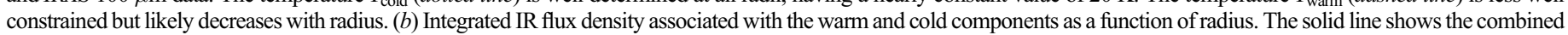
(warm and cold) flux density, in comparison to a comparable result obtained by fitting the dust SED with the DH02 models (DH02, $m a g e n t a)$. $\left(c\right.$ ) Observed ratio of $F_{\mathrm{DUST}}$ to $F_{\mathrm{UV}}$ for

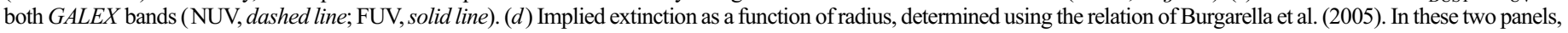

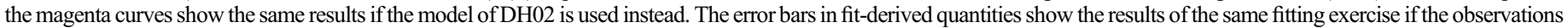
are changed from their nominal value within the uncertainty due to sky subtraction. Additional GALEX zero-point systematic uncertainties are indicated in the right panels.

and Heckman et al. (1998) also showed (in starburst galaxies) that $A(\mathrm{UV})$ and $Z$ are correlated.

Another trend with radius is a progressive blueing of the FUVNUV color (as can be deduced from the profiles of Fig. 4). Together with the decrease of FIR/FUV, this is equivalent to the "IRX- $\beta$ " relationship visible among the integrated galaxies observed by GALEX and is mainly discussed in other current works (e.g., Seibert et al. 2005; Gil de Paz et al. 2006). It has been previously observed in radial profiles of M83 (Boissier et al. 2005), and a sample of the largest galaxies observed with GALEX and IRAS (S. Boissier et al. 2008, in preparation). We discuss below this relation between UV color and FIR/FUV in resolved regions where it might appear more dispersed (e.g., in M51; Calzetti et al. 2005).

\subsection{Stellar Complexes, Star-forming Regions, and Associated Dust Clouds}

Our photometry of UV- and UV+IR-bright features evident on the smallest scales probed by the GALEX and Spitzer data sets has yielded two contrasting samples. They provide a means of understanding selection effects affecting UV-optical-only observations of nearby star-forming galaxies. We note that the UV and UV+IR samples allow us to compare extincted and extinctionfree perspectives of NGC 7331. A complicating factor is that the UV extinction is also expected to depend on the age of a structure (Roussel et al. 2005).

Our FUV-selected sample contained 76 sources appearing as $5 \sigma$ enhancements above the local background in both FUV and NUV GALEX bands. The FUV+IR-selected sample, defined using our "bolometric" image, consisted of 82 sources above the $5 \sigma$ limit in not only FUV and NUV but also $8 \mu \mathrm{m}$ dust and $24 \mu \mathrm{m}$ imagery. We also demanded that the regions in both samples have a minimum final size corresponding to 1.5 times the PSF area. Demanding the same $5 \sigma$ level of significance at 8 and $24 \mu \mathrm{m}$ for the FUV-selected sample cuts the number of UV-bright substructures to 59. Although there are clearly local variations in the surface number density of UV- and UV+IR-selected sources, 


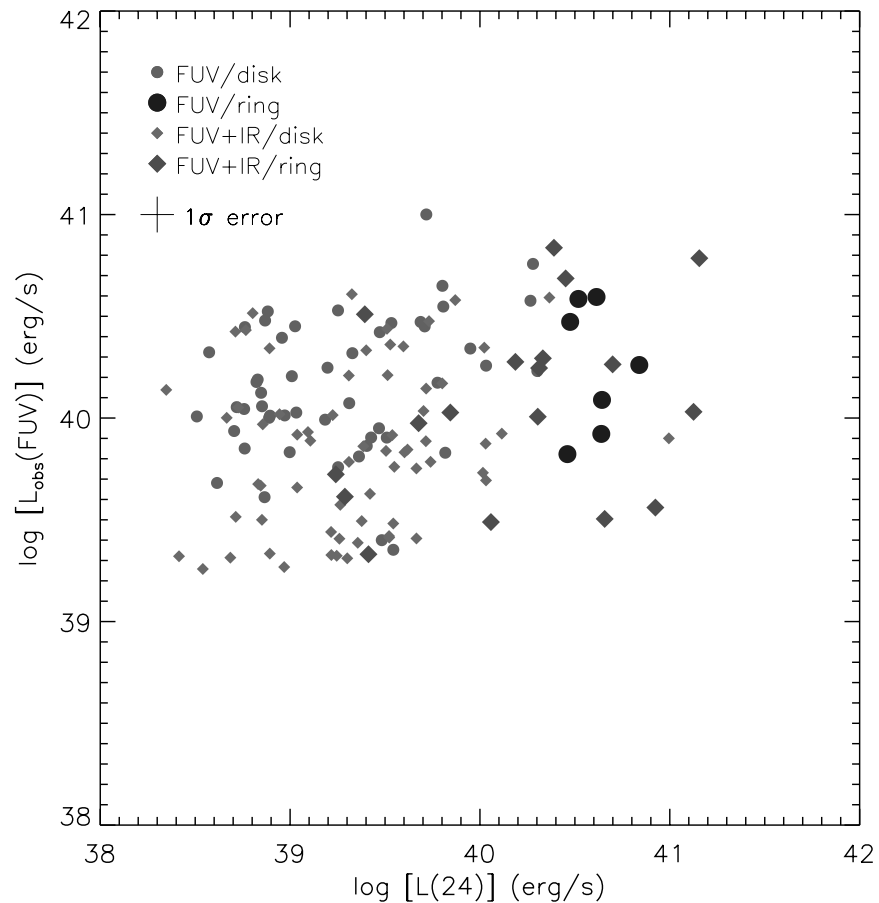

FIG. 7.-Observed FUV luminosity vs. observed $24 \mu \mathrm{m}$ luminosity for UVand UV+IR-selected substructures in NGC 7331. Diamonds represent UV+IRselected objects, whereas filled circles represent UV-selected objects. The symbol size indicates whether or not a given structure is located within NGC 7331's prominent star-forming ring (large) or the remaining portion of the disk (small). The error plotted is the maximum expected for a source barely meeting our $5 \sigma$ significance cut in the plotted bands. The observed scatter is therefore intrinsic and not due to measurement error. [See the electronic edition of the Supplement for a color version of this figure.]

reflecting their concentration along spiral arms (for UV and $\mathrm{UV}+\mathrm{IR}$ ) and in the dust ring (for $\mathrm{UV}+\mathrm{IR}$ ), the distribution of prominent star-forming regions and stellar clusters is relatively uniform throughout the inner disk $\left(<R_{25}\right)$. Outside this limit the number of detections per unit area drops sharply in both bands.

\subsubsection{Comparison of Ultraviolet and Infrared Properties}

In the following analysis, we have separated all detected substructures into "disk" and "ring" populations, with the aim of distinguishing any peculiar characteristics of the ring population. We classified sources based on their position using a slightly smoothed version of the $\mathrm{CO}$ image to estimate the spatial extent of the ring. Furthermore, we have specifically excluded NGC 7331's Seyfert nucleus from all plots.

Figure 7 presents $L_{\text {obs }}($ FUV ) versus $L(24 \mu \mathrm{m})$ for our UV and $\mathrm{UV}+\mathrm{IR}$-selected substructures. One can see that both high-significance ( $5 \sigma$ in all bands) photometric samples become clearly incomplete below limits of $L_{\text {obs }}(\mathrm{FUV}) \sim 10^{39.6} \mathrm{ergs} \mathrm{s}^{-1}$ and $L(24 \mu \mathrm{m}) \sim 10^{38.7} \mathrm{ergs} \mathrm{s}^{-1}$. As described in $\oint 3.2$, the $24 \mu \mathrm{m}$ luminosity is roughly an order of magnitude smaller than the total IR flux from 3 to $1100 \mu \mathrm{m}$. Hence, our samples are populated to about the same limit when comparing the lowest FUV and total IR fluxes. There is no significant correlation between $L_{\mathrm{obs}}(\mathrm{FUV})$ and $L(24 \mu \mathrm{m})$. The apparent scatter is much larger than can be accounted for by measurement errors. Moreover, the level of scatter is variable depending on the sample selection. The range is about $0.8 \mathrm{dex}$ in $L_{\mathrm{obs}}(\mathrm{FUV}) / L(24 \mu \mathrm{m})$ at fixed $L(24 \mu \mathrm{m})$ for the UV-selected sources, whereas the inclusion of highly extincted stellar complexes via UV+IR selection boosts the dispersion to $\sim 1.3$ dex. The scatter in this diagram probably

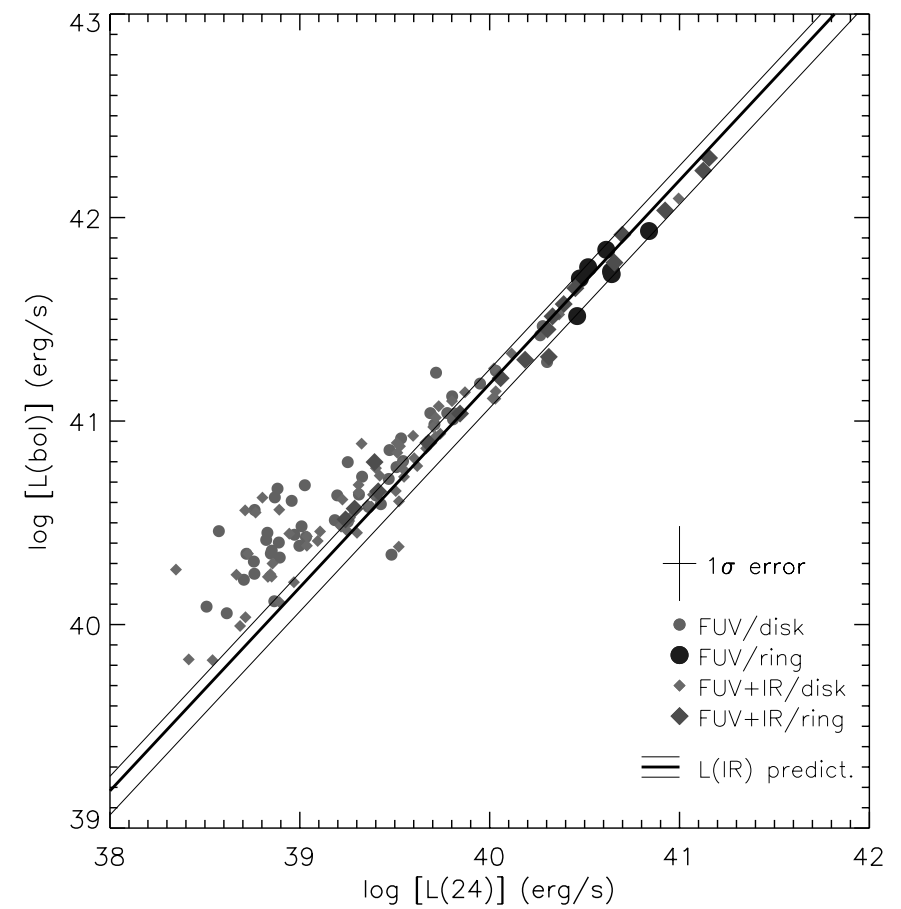

FIG. 8.-Bolometric luminosity, $L(\mathrm{bol})=\nu_{\mathrm{FUV}} L_{\nu, \mathrm{obs}}(\mathrm{FUV})+(1-\eta) L(\mathrm{IR})$, vs. observed $24 \mu \mathrm{m}$ luminosity for UV-and UV+IR-selected substructures in NGC 7331. Symbol assignment is the same as for Fig. 7. The tight correlation between these quantities is primarily a consequence of our method for determining the total IR luminosity at the resolution of our GALEX observations, based on the 8 and $24 \mu \mathrm{m}$ Spitzer imagery alone. Genuine scatter is introduced by the addition of the FUV contribution to the luminosity budget and by variation of the dust properties, which we attempt to trace using the $L_{\nu}(8 \mu \mathrm{m}) / L_{\nu}(24 \mu \mathrm{m})$ term of eq. (1). Our IR prediction for various representative $L_{\nu}(8 \mu \mathrm{m}) / L_{\nu}(24 \mu \mathrm{m})$ values is shown with the triplet of lines and is described in the text. Note the tendency at low luminosities for data points to fall above the predicted range, implying a UV contribution from less attenuated/more evolved stars or possibly even reflecting a secondary UV-bright population on the line of sight. [See the electronic edition of the Supplement for a color version of this figure.]

reflects the combined effects of aging and variable extinction (star/dust geometry) and highlights the difficulty of breaking such a degeneracy. In addition, at the small scales $\left(6^{\prime \prime} \sim 0.4 \mathrm{kpc}\right)$ we are probing, the energy budget is not fully balanced; some fraction of the UV photons likely escape and transfer their energy to dust located elsewhere.

Figure 8 demonstrates a systematic increase in the $L(\mathrm{bol}) /$ $L(24 \mu \mathrm{m})$ ratio at low $L(24 \mu \mathrm{m})$ even for FUV+IR-selected objects. The triplet of lines shown in the diagram, indicates the predicted $L($ IR) computed using equation (1) and assuming three representative values of $\log \left[F_{\nu}(8 \mu \mathrm{m}) / F_{\nu}(24 \mu \mathrm{m})\right]$. The observed, nonlinear behavior reiterates the need for both $I R$ and $U V$ photometry when studying moderate-to-low-luminosity star-forming regions, especially those below $\log L$ (bol) $\left(\mathrm{ergs} \mathrm{s}^{-1}\right)<41$. Note that source aging and geometry will tend to populate the area with an $L($ bol $) / L(24 \mu \mathrm{m})$ excess. Sources with very low attenuation would occupy this regime, as the $L$ (bol) term includes the UV luminosity of the region. In a small number of cases for which $L(\mathrm{bol}) / L(24 \mu \mathrm{m})$ is especially high, some undetermined fraction of the enhancement could be caused by a second (presumably less attenuated than the primary IR emitter) stellar population along the same light of sight. Nevertheless, we use the sum of FUV and IR luminosity to estimate the bolometric energy output of star-forming regions and young stellar complexes traced by our GALEX and Spitzer observations, as outlined in $\S 3.3$. Figure 9 shows the correlation between $L(\mathrm{bol})$ and $L(\mathrm{IR}) / L_{\mathrm{obs}}(\mathrm{FUV})$. Heckman et al. (1998) demonstrated the existence of a relation 


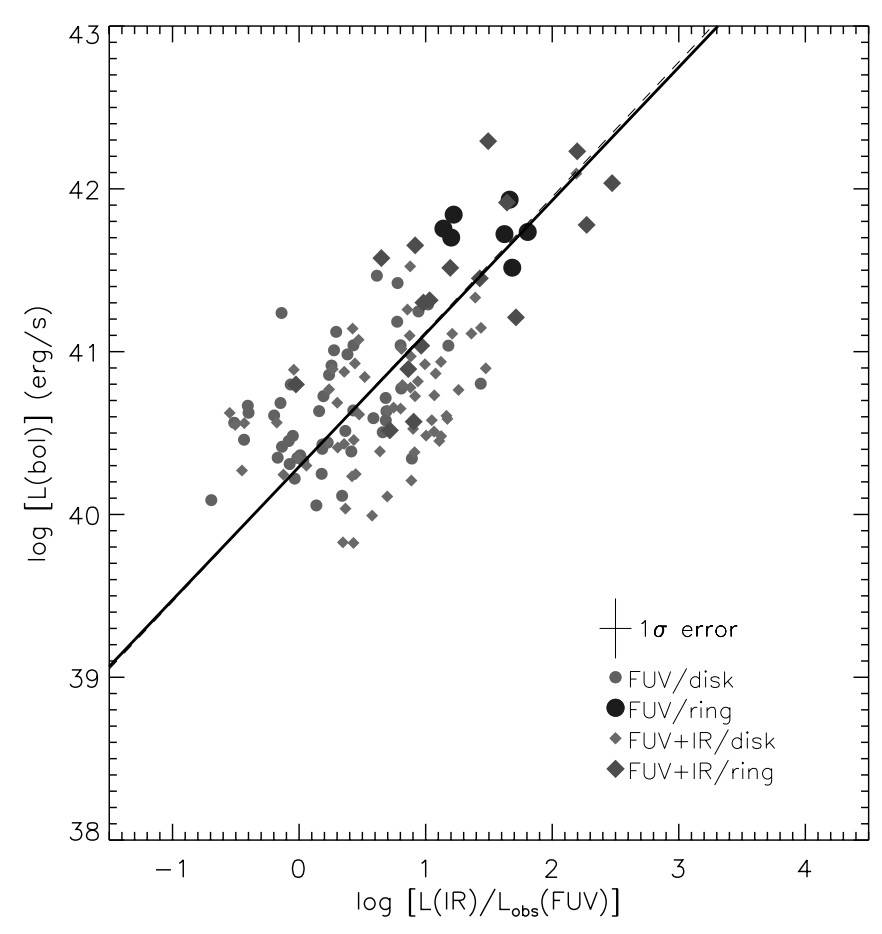

Fig. 9.- Plot of $L\left(\right.$ bol) vs. $L($ IR $) / L_{\text {obs }}($ FUV) for UV- and UV+IR-selected substructures in NGC 7331. Symbol assignment is the same as for Fig. 7. Evidently, the SFR-UV attenuation relationship holds on subgalactic scales in NGC 7331 with nearly the same slope as first established in starbursts. We have plotted a robust linear fit to our data with a solid line and the starburst relationship of Heckman et al. (1998) with a dashed line. The starburst line has been renormalized to lower luminosities by shifting to match our fit at $\log L(\mathrm{IR}) / L_{\mathrm{obs}}(\mathrm{FUV})=0$. The two lines can barely be distinguished as separate. [See the electronic edition of the Supplement for a color version of this figure.]

between SFR and UV attenuation for a sample of starburst galaxies. Photometry obtained for both our UV- and UV+IR-selected samples is consistent with the evidence of Calzetti et al. (2005), which suggests that the correlation holds (at $7 \sigma$ significance according to the Spearman rank test) for SFRs appropriate to subgalactic scales although still above $0.001 M_{\odot} \mathrm{yr}^{-1}$. Fitting our data yields a relationship equivalent to that determined by Heckman et al. (1998), excepting a renormalization to lower luminosity. We find $\log L$ (bol) $\left(\operatorname{ergs~s}^{-1}\right)=40.3+0.82 \log \left[L(\mathrm{IR}) / L_{\text {obs }}(\mathrm{FUV})\right]$ for the substructures of NGC 7331. Note that starburst galaxies show substantially less scatter about this relation, even if the trend is equivalent in slope.

Figure 10 plots $A(\mathrm{FUV})$ versus galactocentric distance for sources in NGC 7331. There is a strong decline in typical extinction with increasing distance, dropping from $\sim 5$ mag in some parts of the star-forming ring to less than 1 mag near $D_{25}$. We note that highly extincted sources are found over the entire disk, even if the typical $A(\mathrm{FUV})$ drops with galactocentric distance. In addition, at the $6^{\prime \prime} \sim 0.4 \mathrm{kpc}$ scales probed by our photometry, local extinction determinations often exceed the corresponding value derived from radial-profile analysis. In Figure 10, the UV-selected "disk" sample is somewhat displaced from the UV+IR-selected sources, showing a bias toward less extincted environments (as expected). Almost three quarters of the UV-selected disk population has $A(\mathrm{FUV})$ less than $1 \mathrm{mag}$.

Much work in recent years has been devoted to exploring the relationship between the UV opacity [ judged via $L(\mathrm{IR}) / L_{\mathrm{obs}}(\mathrm{UV})$ ] and the slope of the UV SED in diverse star-forming environments. This correlation was originally quantified in a series of papers by Calzetti et al. (1994, 1995) and Meurer et al. (1999) based on a sample of starburst galaxies. In these studies the UV slope

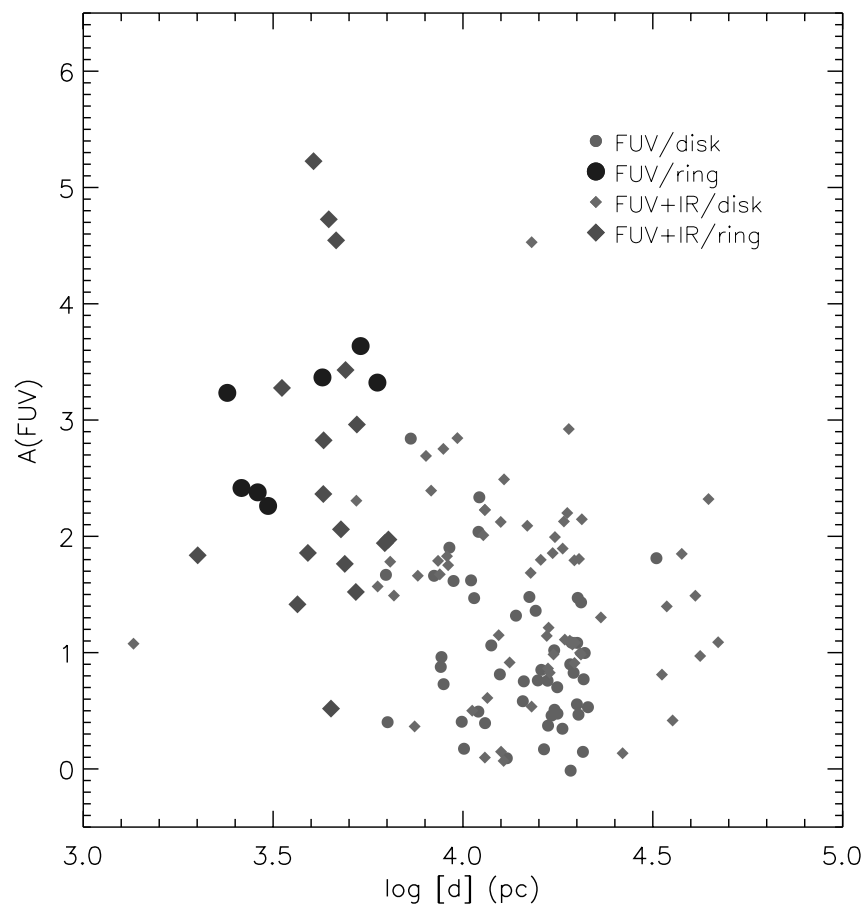

FIG. 10.-FUV extinction, $A(\mathrm{FUV})$, vs. deprojected galactocentric distance for UV- and UV+IR-selected substructures in NGC 7331. Symbol assignment is the same as for Fig. 7. The extinction $A(\mathrm{FUV})$ has been computed from $L(\mathrm{IR}) /$ $L_{\text {obs }}$ (FUV) following the $G A L E X$-specific prescription of Burgarella et al. (2005). There is a general trend toward lower $A(\mathrm{FUV})$ as a function of increasing galactocentric distance, but highly extincted stellar complexes can be found even in the outer portion of the disk. UV selection is obviously biased in identifying the least extincted portions of the true stellar population, which is an aggregate of unobscured and dust-enshrouded clusters. [See the electronic edition of the Supplement for a color version of this figure.]

came to be expressed as $\beta$, with Calzetti et al. (1994) defining $\beta_{26}$ specifically over the $0.13-0.26 \mu \mathrm{m}$ wavelength range. Bell et al. (2002) showed that "quiescent" (although still star-forming) galaxies follow a different track in the $L($ IR $) / L_{\text {obs }}(\mathrm{UV})-\beta$ plane, offset to redder UV slopes (higher relative to starburst galaxies having comparable opacity). Goldader et al. (2002) examined a sample of ultraluminous IR galaxies (ULIRGs) and demonstrated they also deviate from the starburst galaxy opacity-reddening relationship. Bell (2002) examined possible causes for such an offset, concluding that changes in dust geometry and properties are most likely the underlying cause with a possible contribution to the intrinsic UV color originating from older stellar populations. Kong et al. (2004) attempted to ascribe the displacement and broadening of the UV opacity- $\beta$ relationship to changes in SFH, parameterized in the form of $b$. Using GALEX and IRAS observations for a sample of more than 400 galaxies, Seibert et al. (2005) began ongoing efforts to further constrain a possible "secondary parameter" and tighten the relationship for galaxies of all types.

Figure 11 presents $L(\mathrm{IR}) / L_{\mathrm{obs}}(\mathrm{UV})$ versus $L_{\lambda, \mathrm{obs}}(\mathrm{FUV}) /$ $L_{\lambda \text {,obs }}$ (NUV) (gauging the UV slope) for the substructures of NGC 7331. We can use the data in Figure 11 to discriminate between possible dust models. In the figure we have overplotted curves representing the predicted relationship of $L(\mathrm{IR}) / L_{\mathrm{obs}}(\mathrm{FUV})$ to UV slope for various types (Milky Way, Small Magellanic Cloud [SMC], starburst) and geometries (mixed, foreground screen) of dust, adopting a constant SFR as a first-order stellar population. Many of the NGC 7331 sources appear to be consistent with Milky Way dust configured in a mixed star/dust geometry (left dashed line). This statement rests on the fact that many of the sources lie at bluer $L_{\lambda, \mathrm{obs}}(\mathrm{FUV}) / L_{\lambda, \mathrm{obs}}(\mathrm{NUV})$ than allowed for by the starburst 


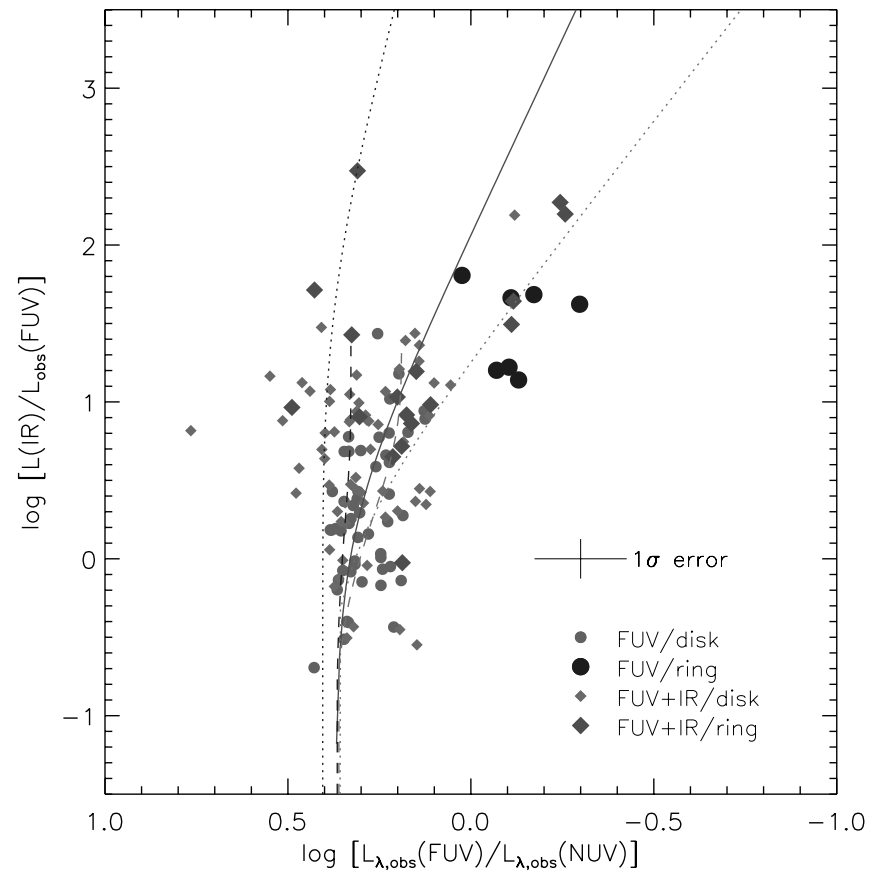

FIG. 11.-Plot of $L\left(\right.$ IR) $/ L_{\text {obs }}\left(\right.$ FUV) vs. $L_{\lambda, \text { obs }}(\mathrm{FUV}) / L_{\lambda, \text { obs }}(\mathrm{NUV})$ for UVand UV+IR-selected substructures in NGC 7331. Symbol assignment is the same as for Fig. 7. The lines represent model predictions for a continuously forming stellar population, in the case of various prescriptions for dust composition and star/dust geometry. The dust models are fully described in the text. Left lines are for Milky Way-type dust (mixed, dashed line; screen, dotted line), right lines are for SMC-type dust (mixed, dashed line; screen, dotted line), and the solid curve is specifically applicable to starburst galaxies (Calzetti law). The quantity $A$ (FUV) increases with $L(\mathrm{IR}) / L_{\mathrm{obs}}(\mathrm{FUV})$ along each of the model curves. The starburst and screen models span the entire range of $A(\mathrm{FUV})$ in the plot (from vanishingly small to $8 \mathrm{mag}$ ), whereas the mixed models "saturate" at $A(\mathrm{FUV})=2.75 \mathrm{mag}$. The disk data points appear most consistent with the Milky Way-type dust, with the possible exception of some star-forming ring sources which may be starburstlike. Again, our models are for continuous star formation. If we had instead plotted predictions for aging instantaneous bursts, the model curves would shift progressively to the right with age. [See the electronic edition of the Supplement for a color version of this figure.]

and SMC attenuation laws. This would be even more the case had we modeled aging instantaneous bursts rather than continuous star formation, as the model tracks would shift to redder UV colors. Furthermore, the relative lack of data points at larger $L$ (IR) $/ L_{\text {obs }}$ (FUV) than predicted by the Milky Way/mixed models suggests that a foreground screen is not the best description for disk sources outside of the star-forming ring. It is important to note that this could partially be a stochastic effect as we expect few of the most active (hence heavily attenuated) sources in relation to the number of average sources. Regardless, it appears that Milky Way-type dust is appropriate for a large fraction of our sample. Independent of the population-synthesis models in Figure 11 , the very weak dependency between $L($ IR $) / L_{\text {obs }}($ FUV $)$ and the FUV-NUV color already suggests Milky Way-type dust [since $A(\mathrm{FUV}) / A(V) \sim A(\mathrm{NUV}) / A(V)$ for Milky Way-type dust] or the importance of radiative transfer effects acting to flatten the UV spectrum.

However, there are several sources found at rather high $L(\mathrm{IR}) /$ $L_{\text {obs }}(\mathrm{FUV})$ and moderate $L_{\lambda, \mathrm{obs}}(\mathrm{FUV}) / L_{\lambda, \mathrm{obs}}(\mathrm{NUV})$; these are typically "ring" star-forming regions. Given that we know they are not highly aged due to the $\mathrm{H} \alpha$ emission from the ring, we suggest that the ring may be best characterized by the starburst attenuation law, rather than by a Milky Way curve. These sources, with the lowest $L_{\lambda, \text { obs }}(\mathrm{FUV}) / L_{\lambda, \text { obs }}(\mathrm{NUV})$, are generally also the

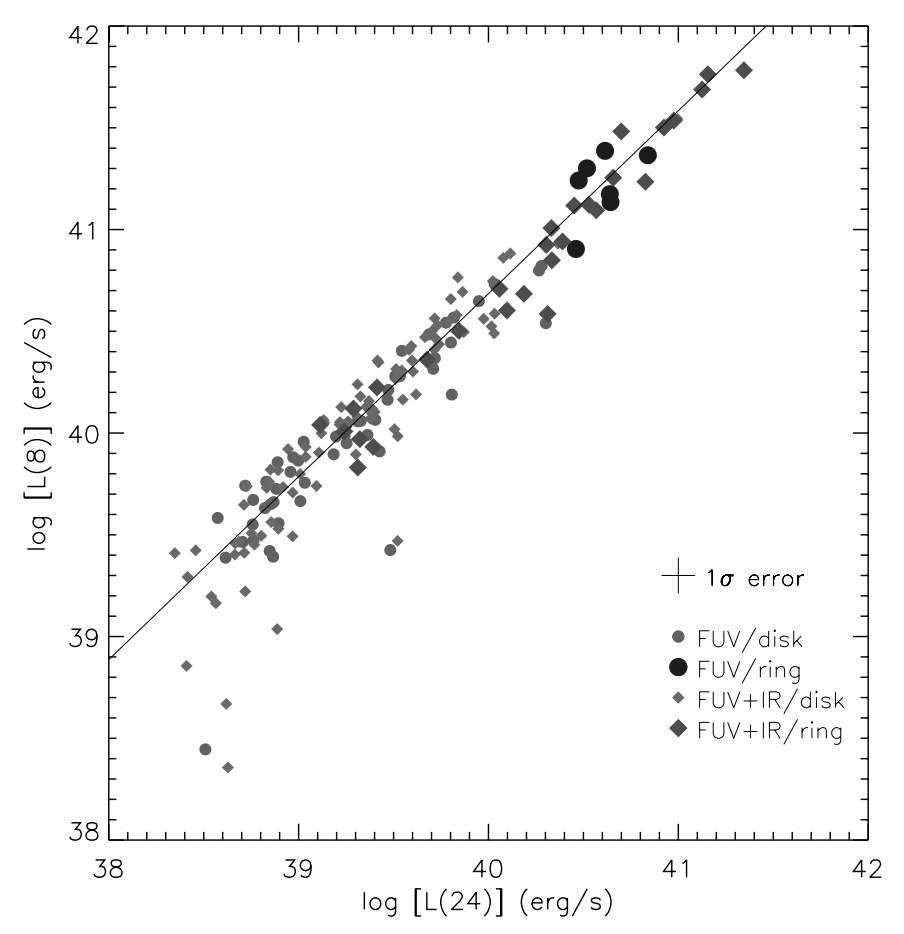

FIG. 12.-Observed $L(8 \mu \mathrm{m})$ vs. $L(24 \mu \mathrm{m})$ for UV- and UV+IR-selected substructures in NGC 7331. Symbol assignment is the same as for Fig. 7. We overplot a robust linear fit to our photometry. The (log-log) slope of the best-fit relation is 0.90 , slightly less than unity. The high degree of correlation could perhaps justify use of $L(24 \mu \mathrm{m})$ alone to estimate $L($ IR) in galaxies for which IRAC $8 \mu \mathrm{m}$ data do not exist, if a well-founded model incorporating both environmental conditions and source luminosity could be developed on the basis of a large galaxy sample. [See the electronic edition of the Supplement for a color version of this figure.]

most luminous in the galaxy [as expected based on their high $\left.L(\mathrm{IR}) / L_{\text {obs }}(\mathrm{FUV})\right]$.

\subsubsection{8 and $24 \mu \mathrm{m}$ Properties}

Figure 12 plots the observed nonstellar (dust-only) emission at $8 \mu \mathrm{m}$ as a function of the integrated $24 \mu \mathrm{m}$ luminosity for each highly significant star-forming region or stellar complex in NGC 7331 (at $0.4 \mathrm{kpc}$ resolution). The two quantities are highly correlated over 3 orders of magnitude in luminosity. A robust linear fit to the data in Figure 12 yields a relation given by $\log L(8 \mu \mathrm{m})\left(\operatorname{ergs~s}^{-1}\right)=4.72+0.90 \log L(24 \mu \mathrm{m})\left(\operatorname{ergs~s}^{-1}\right)$. The slope of the relation is slightly less than unity, as also found in M51 by Calzetti et al. (2005). We note that the rms scatter about the fit depicted in Figure 12 is remarkably small, amounting to 0.13 dex for $L(24 \mu \mathrm{m})>10^{39} \mathrm{ergs} \mathrm{s}^{-1}$.

If the 8 and $24 \mu \mathrm{m}$ luminosity of dust clouds tracing starforming regions and stellar complexes on the scale of $\sim 0.4 \mathrm{kpc}\left(6^{\prime \prime}\right)$ are so highly correlated, would this correlation remain on even smaller scales (such as the $150 \mathrm{pc}\left[2.1^{\prime \prime}\right]$ traced by the $8 \mu \mathrm{m}$ dustonly image)? We know the correlation does break down at the limit of individual star-forming regions, given the rather different morphology for the bands of interest in NGC 300 (Helou et al. 2004), with $24 \mu \mathrm{m}$ emission being more centrally concentrated on $\mathrm{H}$ II regions than $8 \mu \mathrm{m}$, evaluated at $\sim 60 \mathrm{pc}$ resolution. It seems critical, therefore, to place concentrated effort on analyzing galaxies nearer than an intermediate distance limit of $\sim 4-5 \mathrm{Mpc}$ $\left(6^{\prime \prime}=120-150 \mathrm{pc}\right)$ in order to gain morphological clues regarding systematic variation of $L_{\nu}(8 \mu \mathrm{m}) / L_{\nu}(24 \mu \mathrm{m})$.

Global studies of $L_{\nu}(8 \mu \mathrm{m}) / L_{\nu}(24 \mu \mathrm{m})$ for a variety of integrated metallicities have demonstrated a clear dependence of the ratio on $Z$, with a distinct shift to lower $L_{\nu}(8 \mu \mathrm{m}) / L_{\nu}(24 \mu \mathrm{m})$ 

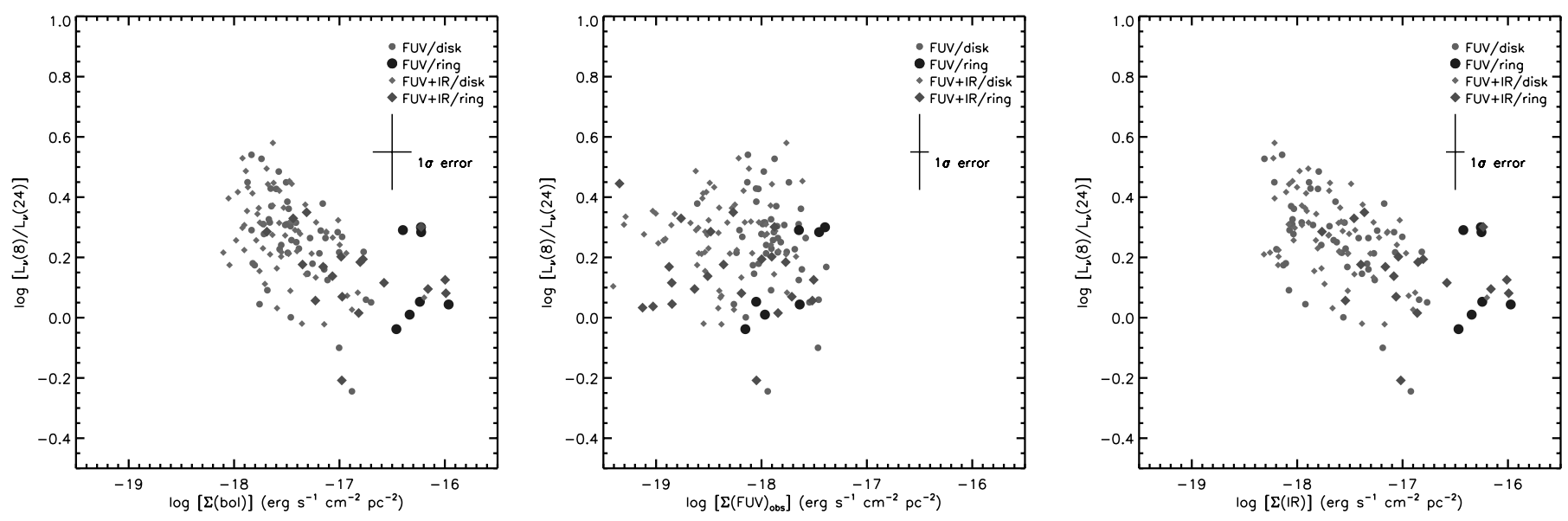

Fig. 13. - Left: The $L_{\nu}(8 \mu \mathrm{m}) / L_{\nu}(24 \mu \mathrm{m})$ vs. $\Sigma$ (bol) for UV- and UV+IR-selected substructures in NGC 7331. Symbol assignment is the same as for Fig. 7. The quantity $L_{\nu}(8 \mu \mathrm{m}) / L_{\nu}(24 \mu \mathrm{m})$ is anticorrelated with bolometric surface brightness, such that more luminous sources tend to have a lower $8 \mu \mathrm{m} / 24 \mu \mathrm{m} \mathrm{ratio}$. This result suggests that the stimulation of PAH emission is strongly dependent on the intensity of the local UV radiation field, although it has been suggested that metallicity is also important. Center: The $L_{\nu}(8 \mu \mathrm{m}) / L_{\nu}(24 \mu \mathrm{m})$ versus $\Sigma(\mathrm{FUV})_{\text {obs. }}$. The quantity $L_{\nu}(8 \mu \mathrm{m}) / L_{\nu}(24 \mu \mathrm{m})$ is uncorrelated with the observed FUV surface brightness, although a correlation is recovered if the data points are corrected for our estimated $A(\mathrm{FUV})$ per source. Right: The $L_{\nu}(8 \mu \mathrm{m}) / L_{\nu}(24 \mu \mathrm{m})$ versus $\Sigma(\mathrm{IR})$. A strong correlation is present, implying that obscured star formation activity is what drives the relation seen in Fig. 14 (left) vs. $\Sigma$ (bol). [See the electronic edition of the Supplement for a color version of this figure.]

below a limiting metallicity (Engelbracht et al. 2005). In the same way, the progressive diminishment of $L_{\nu}(8 \mu \mathrm{m}) / L_{\nu}(24 \mu \mathrm{m})$ with increasing $L_{\nu}(24 \mu \mathrm{m})$ probably reflects an underlying change in the dust composition of diverse star formation environments, in addition to the simple heating associated with more intense star formation.

Calzetti et al. (2005) showed that the most active star-forming regions of M51's disk have lower $L_{\nu}(8 \mu \mathrm{m}) / L_{\nu}(24 \mu \mathrm{m})$, even when the SFR density is judged from extinction-corrected $\mathrm{H} \alpha$ luminosity, $L_{\text {corr }}(\mathrm{H} \alpha)$, without any adjustment for the relative surface covering factor of $\mathrm{H}$ in nebulae within the aperture. Figure 13 (left) presents the $L_{\nu}(8 \mu \mathrm{m}) / L_{\nu}(24 \mu \mathrm{m})$ measured for each UV- and UV+IR-selected structure within NGC 7331 as a function of $\Sigma$ (bol), a proxy for extinction-free SFR per unit disk area. The stellar contribution to the $8 \mu \mathrm{m}$ band has been removed as described in Appendix A. A strong correlation is observed, with the typical $L_{\nu}(8 \mu \mathrm{m}) / L_{\nu}(24 \mu \mathrm{m})$ value decreasing by a factor of $\sim 3$ over an order-of-magnitude increase in $\Sigma($ bol). Most of this correlation is driven by the observed IR luminosity, rather than any UV contribution, as can be seen in Figures 13 (center and right), which plot $L_{\nu}(8 \mu \mathrm{m}) / L_{\nu}(24 \mu \mathrm{m})$ versus $\Sigma(\mathrm{FUV})_{\mathrm{obs}}$ and $\Sigma($ IR), respectively. Without correction for extinction, the average FUV surface brightness of a substructure is uncorrelated with $L_{\nu}(8 \mu \mathrm{m}) / L_{\nu}(24 \mu \mathrm{m})$. The strong dependence of $L_{\nu}(8 \mu \mathrm{m}) /$ $L_{\nu}(24 \mu \mathrm{m})$ on $\Sigma(\mathrm{IR})$, but not observed $\Sigma(\mathrm{FUV})$, is to be expected as the MIR-FIR emission (in all bands) has a common origin within the cocoon of natal dust, whereas the observed UV emission can originate both inside and outside this environment, with the internal component being highly attenuated in general. Note that after correction for extinction, the $\Sigma(\mathrm{FUV})_{\text {corr }}$ does correlate with $L_{\nu}(8 \mu \mathrm{m}) / L_{\nu}(24 \mu \mathrm{m})$.

Correcting our $\mathrm{H} \alpha$ photometry for extinction (as explained in $\S$ 4.4.2) and expressing these measurements in terms of surface brightness, we can probe the influence of star formation on $L_{\nu}(8 \mu \mathrm{m}) / L_{\nu}(24 \mu \mathrm{m})$ over even shorter timescales. Figure 14 (left) illustrates the dependence of $L_{\nu}(8 \mu \mathrm{m}) / L_{\nu}(24 \mu \mathrm{m})$ on $\Sigma(\mathrm{H} \alpha)_{\text {corr }}$ In Figure 14 (left) we overplot a fit to all substructures (both UV and $\mathrm{UV}+\mathrm{IR}$ selected). The parameters are $\log L_{\nu}(8 \mu \mathrm{m}) / L_{\nu}(24 \mu \mathrm{m})=$ $-6.46-0.33 \log \Sigma(\mathrm{H} \alpha)_{\mathrm{corr}}\left(\mathrm{ergs} \mathrm{s}^{-1} \mathrm{~cm}^{-2} \mathrm{pc}^{-2}\right)$. Given that the UV radiation field experienced by PAH carriers does in fact change on the timescale of a few megayears ( probed by $\mathrm{H} \alpha$ ) and that the $\mathrm{O}$ stars producing the $\mathrm{H}$ II emission should be the most effective at destroying PAH molecules (compared to B stars), we suggest the $\Sigma(\mathrm{H} \alpha)_{\text {corr }}$ versus $L_{\nu}(8 \mu \mathrm{m}) / L_{\nu}(24 \mu \mathrm{m})$ relation is preferred over the similar IR+UV-, IR-, or UV-based results. But what are the remaining variables in a viable model for the local $L_{\nu}(8 \mu \mathrm{m}) /$ $L_{\nu}(24 \mu \mathrm{m})$ value?

We examined the influence of metallicity on the relative strength of PAH and thermal dust emission, obtaining a surprising result. Figure 14 (center) shows the variation of $L_{\nu}(8 \mu \mathrm{m}) / L_{\nu}(24 \mu \mathrm{m})$ as a function of $Z$, taken from Dutil \& Roy (1999). The data suggest a decline in $L_{\nu}(8 \mu \mathrm{m}) / L_{\nu}(24 \mu \mathrm{m})$ at higher metallicity, contrary to the known dependence for integrated galactic measurements. However, the metallicity range $(8.6<Z<9.3)$ probed by our photometry of NGC 7331 star-forming regions is well above the value ( $Z=8.2$; Engelbracht et al. 2005) associated with a shift to categorically lower $L_{\nu}(8 \mu \mathrm{m}) / L_{\nu}(24 \mu \mathrm{m})$. Indeed, over a similar range of $Z$, the galaxies of Engelbracht et al. span an order-of-magnitude range in $L_{\nu}(8 \mu \mathrm{m}) / L_{\nu}(24 \mu \mathrm{m})$ and show no sign of correlation with $Z$. We are unaware of any other study which has addressed the relation between $L_{\nu}(8 \mu \mathrm{m}) / L_{\nu}(24 \mu \mathrm{m})$ and metallicity on the scales we are considering, making it difficult to assess the veracity of our rather unexpected result. Further analysis of the SINGS database is the means to confirm or reject the trend. Numerous galaxies within the sample have the corollary abundance data required to accomplish such a check.

At present, we see two possible ways around the apparent contradiction. First, it may be that $\Sigma(\mathrm{H} \alpha)_{\text {corr }}$ is the dominant factor in estimating PAH strength once $Z$ is above a certain threshold, overwhelming the local variation of $Z$ in environments of supersolar $Z$. In NGC 7331, the existence of the bright star-forming ring at small radii characterized by relatively high metallicity could force a plot such as Figure 14 (center) to mimic a decline in $L_{\nu}(8 \mu \mathrm{m}) / L_{\nu}(24 \mu \mathrm{m})$ caused by progressively higher $Z$. In Figure 14 (right) we look into this possibility, plotting the bivariate distribution $\left(\Sigma(\mathrm{H} \alpha)_{\text {corr }}, Z\right)$ of sources in a small number of fixedwidth bins of $\log L_{\nu}(8 \mu \mathrm{m}) / L_{\nu}(24 \mu \mathrm{m})$. Each bin was assigned to a different symbol color. If the local SFR density significantly dominated metallicity, we would expect to see a strong gradient in symbol color along the $\Sigma(\mathrm{H} \alpha)_{\text {corr }}$ axis, with little systematic 

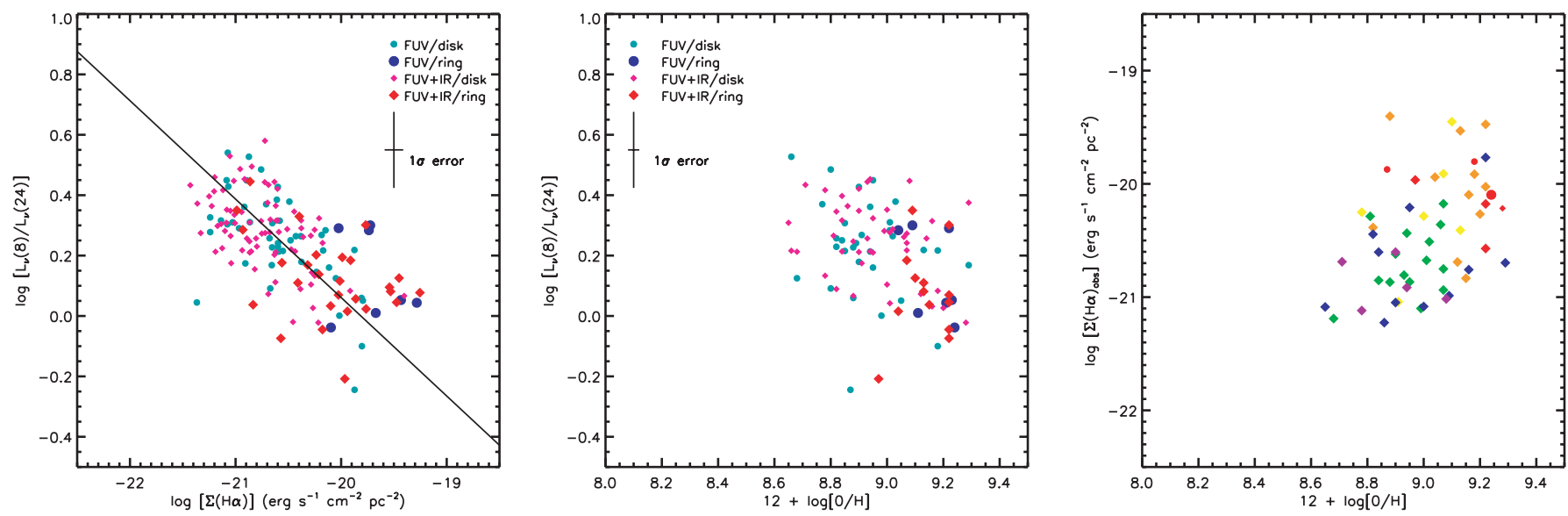

FIG. 14. - Left: Plot of $L_{\nu}(8 \mu \mathrm{m}) / L_{\nu}(24 \mu \mathrm{m})$ vs. $\Sigma(\mathrm{H} \alpha)_{\text {corr }}$ for UV- and UV+IR-selected substructures in NGC 7331. Symbol assignment is the same as for Fig. 7. The $\mathrm{H} \alpha$ surface brightness has been extinction corrected with a Milky Way extinction law, to a degree such that the measured $A(\mathrm{FUV})$ is met on a source-by-source basis. The observed correlation is best fit by a relation of the following form: $\log L_{\nu}(8 \mu \mathrm{m}) / L_{\nu}(24 \mu \mathrm{m})=-6.46-0.33 \log \Sigma(\mathrm{H} \alpha)_{\text {corr }}$, shown by the solid line in this plot. Center: $L_{\nu}(8 \mu \mathrm{m}) /$ $L_{\nu}(24 \mu \mathrm{m})$ vs. metallicity, $Z$. An apparent correlation is present, but in the opposite sense of what we expected. Higher metallicity sources appear to have smaller $L_{\nu}(8 \mu \mathrm{m}) /$ $L_{\nu}(24 \mu \mathrm{m})$. We suspect that the local UV radiation field dominates the influence of metallicity on these relatively small scales $(\sim 0.4 \mathrm{kpc})$. Indeed, the sources having the highest SFR surface density are also located in high-metallicity areas, like NGC 7331's star-forming ring. Right: Distribution of sources having varied $L_{\nu}(8 \mu \mathrm{m}) / L_{\nu}(24 \mu \mathrm{m})$ in the $\left(\Sigma(\mathrm{H} \alpha)_{\text {corr }}, Z\right)$ plane. The points are color-coded according to the following scale of $R_{8,24}=L_{\nu}(8 \mu \mathrm{m}) / L_{\nu}(24 \mu \mathrm{m}): R_{8,24}<0$ (red), $0<R_{8,24}<0.1$ (orange), $0.1<$ $R_{8,24}<0.2$ (yellow), $0.2<R_{8,24}<0.3$ (green), $0.3<R_{8,24}<0.4$ (blue), and $0.4<R_{8,24}$ ( purple).

change in the color of points as a function of $Z$ for small intervals in $\Sigma(\mathrm{H} \alpha)_{\text {corr }}$. We do find an obvious gradient with $\Sigma(\mathrm{H} \alpha)_{\text {corr }}$, but note that sources also appear to have increased $L_{\nu}(8 \mu \mathrm{m}) / L_{\nu}(24 \mu \mathrm{m})$ at higher $Z$ even for similar $\Sigma(\mathrm{H} \alpha)_{\text {corr }}$, albeit with much scatter. Another scenario possibly capable of reconciling the integrated and local estimates (of relative PAH strength vs. metallicity) is simply that the integrated measurements include a contribution from the diffuse medium and therefore are not truly representative of the $L_{\nu}(8 \mu \mathrm{m}) / L_{\nu}(24 \mu \mathrm{m})$ in star-forming regions. This seems a bit problematic as it would seemingly require a large (and possibly variable) diffuse fraction for the IR emission in a star-forming galaxy. Although such fractions have yet to be quantified, visual inspection of the Spitzer data for the SINGS sample yields an impression that diffuse emission, although certainly present, is not so prevalent energetically.

\subsubsection{Extinction-free Schmidt-Law and Star Formation Thresholds}

Schmidt (1959) first proposed a power-law correlation between the SFR and gas mass density, $\rho$. Due to the difficulty of estimating volume densities in external galaxies, the idea was eventually recast into terms of SFR and gas surface densities (e.g., Sanduleak 1969; Kennicutt 1989; Dopita \& Ryder 1994). Such a power-law relation between the two quantities $\left(\Sigma_{\mathrm{SFR}} \propto \Sigma_{\text {gas }}^{n}\right)$ has been confidently established on galaxy-wide scales (e.g., Kennicutt 1998a) and increasingly toward smaller dimensions (Rownd \& Young 1999; Wong \& Blitz 2002; Boissier et al. 2003; Heyer et al. 2004), although one does not expect the same law to hold on global and local scales, especially in regimes strongly affected by star formation thresholds. Recent theoretical modeling ( $\mathrm{Li}$ et al. 2006; Krumholz \& McKee 2005; Schaye 2004; Mac Low \& Klessen 2004; Kravtsov 2003; Elmegreen 2002) has brought insight concerning the physical basis of the scaling relation and star formation thresholds. However, there remains scarce agreement in the details or precise applicability of the Schmidt-law relations. For instance, is it most appropriate to evaluate the ISM in terms of the total gas surface density or molecular gas alone? What is the limiting scale size below which the local Schmidt law breaks down due to feedback or stochastic effects? What is the optimal tracer of SFR in the Schmidt-law context? For instance, UV and $\mathrm{H} \alpha$ emission probe rather different timescales in the context of an instantaneous starburst event (because $\mathrm{H} \alpha$-emitting $\mathrm{H}$ II is ionized mainly by $\mathrm{O}$ stars, while UV luminosity is dominated by longer lived $\mathrm{B}-\mathrm{A}$ stars).

The uncertainty described above has been sustained because observational studies have generally relied on a censored view of the star formation activity and/or gaseous content. Frequently the SFR in Schmidt-law work is gauged using $\mathrm{H} \alpha$ observations, sometimes uncorrected for the bias of differential extinction among the data set (global or local). This problem is only compounded by the correlation between attenuation and SFR; the locales underrepresented the most are generally the dominant sites of activity. Wong \& Blitz (2002) explicitly show that correcting for extinction to first order (in a radial sense) has the effect of raising the inferred power-law index of the local Schmidt law. Regarding gas content, the number of galaxies for which high-resolution CO imagery is available is dramatically smaller than the sample that have been mapped in $\mathrm{H}$ I. Furthermore, the $\mathrm{CO}$ data which do exist for nearby galaxies are not usually sensitive enough to accurately constrain the distribution of molecular gas surface density in all parts of the disk but generally just the central portion and the CO-bright, moleculeladen spiral arms.

The unique opportunity provided by Spitzer and GALEX to jointly recover the majority of the bolometric luminosity from star-forming regions allows a useful check on the proportionality between gas surface density and the average SFR. In Figure 15a, we plot azimuthally averaged estimates of $\Sigma_{\text {SFR }}$ derived from our profile analysis versus the total gas surface density, $\Sigma_{\text {gas }}=$ 1.36 $\left(\Sigma_{\mathrm{H}_{\mathrm{I}}}+\Sigma_{\mathrm{H}_{2}}\right)$, where the $\mathrm{H}_{\mathrm{I}}$ and $\mathrm{H}_{2}$ measures have been corrected for the inclination of NGC 7331. The factor of 1.36 accounts for the contribution of He to the total gaseous mass surface density. Several tracers of the SFR are shown in Figure 15a. We include the total IR, FUV, $\mathrm{H} \alpha$, and $22 \mathrm{~cm}$ data. For comparison against our radially evaluated results, we overplot the global Schmidt-law relation of Kennicutt (1998a) with an orange dashdotted line. The three black dotted lines indicate gas-consumption timescales of $0.1,1.0$, and 10 Gyr. We have plotted two representations of the local star formation threshold gas surface density of Schaye (2004) using green dashed lines. One has been computed for a characteristic metallicity of $0.5 Z_{\odot}$ and the other for $0.1 Z_{\odot}$. The $0.5 Z_{\odot}$ value was anticipated to be appropriate just 


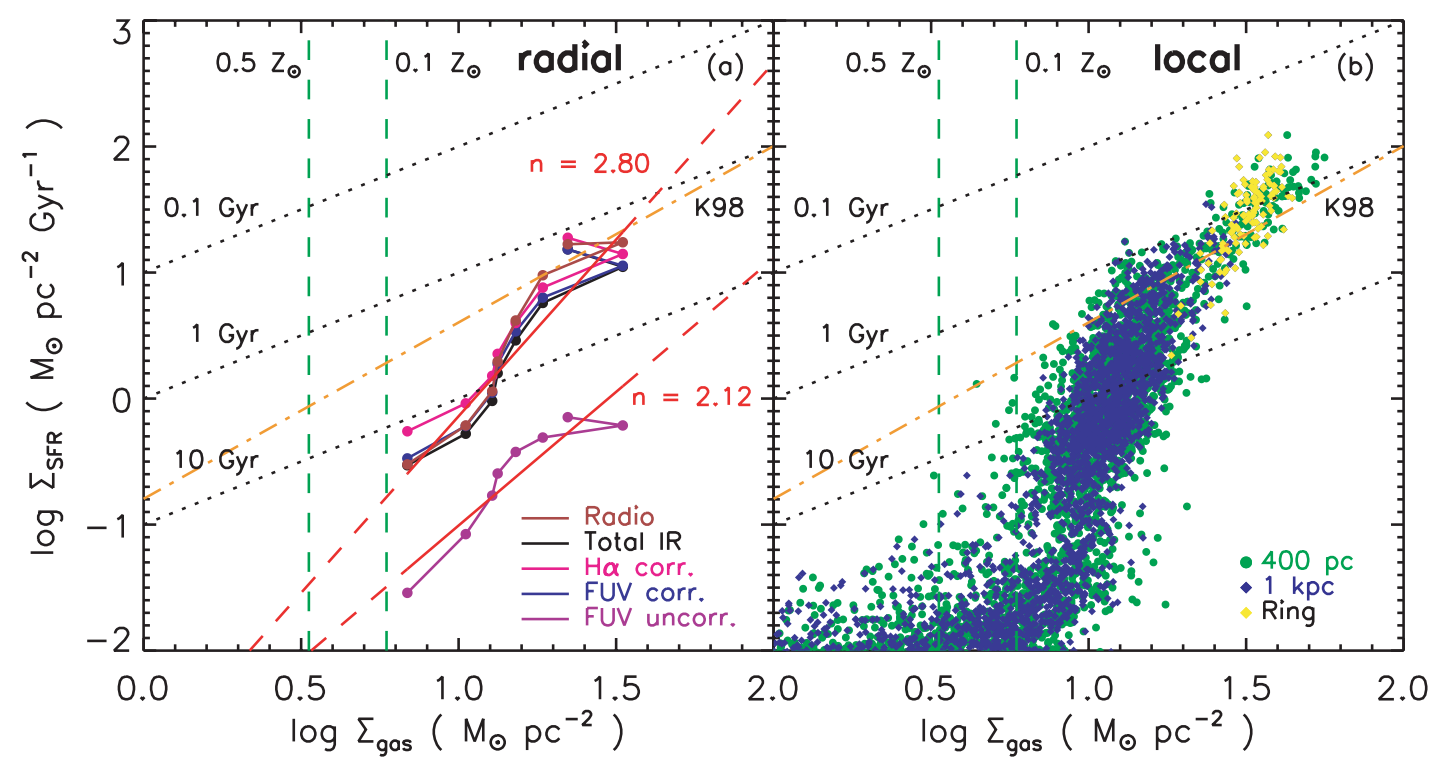

FIG. 15.-Schmidt law in NGC 7331. (a) $\Sigma_{\text {SFR }}$, estimated using various tracers as indicated in the legend, vs. total gas surface density $\left(\Sigma_{\text {gas }}\right)$. The orange dash-dotted line indicates the best-fit relation from Kennicutt (1998a) based on integrated measurements for a large sample of galaxies. The dotted lines correspond to no-recycling gasconsumption timescales of $0.1,1$, and $10 \mathrm{Gyr}$. The red lines are our fits to the observed FUV and IR-based data points. The plot shows that the radial Schmidt law is steeper after accounting for extinction. (b) Local Schmidt law for independent lines of sight in NGC 7331, using only the SFR(bol) indicator. The green points present our data at $0.4 \mathrm{kpc}$ resolution. Blue points show that smoothing the gas-phase data to $1 \mathrm{kpc}$ resolution reduces the scatter in the relationship, as expected if the star formation is disturbing the gas distribution. The yellow points are simply the blue (smoothed) data for pixels found in the star-forming ring. The green dashed lines show the Schaye (2004) local star formation threshold for various metallicities.

inside the $D_{25}$ radius on the basis of the Dutil \& Roy (1999) H II region measurements. However, abundances of $0.1 Z_{\odot}$ are commonly found in the outer spiral disks where star formation is progressively less common.

A few points deserve comment regarding Figure 15a. According to the convention of previous Schmidt-law studies, the data in Figure 15a constitute the basis for a "local" (rather than "global") law. While it is true that the azimuthal averaging process retains more information about inner workings of the galaxy than a global average, subsequently fitting a power law to the radial-profile-based data points essentially ignores the expected nonlinear (Kravtsov 2003) form of the relationship between $\Sigma_{\text {SFR }}$ and $\Sigma_{\text {gas }}$. Fits of this type (e.g., Wong \& Blitz 2002; Fig. 15a, red lines) are bound to typically result in higher power-law indices, unless the points constraining the fit are known to exclude locations susceptible to star formation threshold effects. This is not meant to suggest that fits of the variety presented in Figure $15 a$ are not useful (for galaxy modeling as an example), but rather that they need to be interpreted with care. For this reason we do not refer to the results of Figure $15 a$ as probing the "local" Schmidt law but instead as representing a "radial" parameterization of the Schmidtlaw observables.

Consistent with Wong \& Blitz (2002), we find a significantly steeper radial power-law index after correcting for extinction ( $2.7 \pm 0.4$ vs. $2.1 \pm 0.3$ for FUV) or just by using an extinctionfree SFR tracer (IR, $2.8 \pm 0.4$ ). All of the dust-corrected SFR tracers ( $\mathrm{H} \alpha, \mathrm{FUV})$ and extinction-free SFR tracers (IR, $22 \mathrm{~cm}$ radio continuum) indicate similarly steep Schmidt-law indices. However, as noted above, these indices do not accurately reflect the local, nonlinear Schmidt law on subgalactic scales. The last point to be made from Figure $15 a$ is that there does not appear to be a pending shortage of gas, at any radii, despite our previous result $(\S 4.3)$ that $b_{8}$ for galactocentric distances $\gtrsim 12 \mathrm{kpc}\left(171^{\prime \prime}\right)$ is nearly an order of magnitude greater than in the galaxy center.

In order to probe the complete range of environments in NGC 7331 , we also examined the Schmidt law at the $\sim 0.4 \mathrm{kpc}\left(6^{\prime \prime}\right)$ resolution limit of our combined Spitzer+GALEX FUV+IR image and gaseous tracers. Figure $15 b$ presents data extracted from the high-resolution datacube described in $\S 3.4$. We subsampled the cube every $6^{\prime \prime}$ to ensure that all the points plotted in Figure $15 b$ are independent of each other. The green circles in the figure are the observed data after subsampling, whereas blue triangles represent the gas surface density after smoothing to $1 \mathrm{kpc}\left(14^{\prime \prime}\right)$ resolution (and then subsampling). The $\Sigma_{\mathrm{SFR}}$ in Figure $15 b$ (and in later in Fig. 16) is estimated bolometricly (using our UV+IR formulation). Feedback between massive stars and the spatial distribution of the ISM is expected to be important on small scales, with the creation of superbubbles (e.g., Oey 2004) as one prominent manifestation. Our data (as presented in Fig. 15b) are consistent with this view. In particular, a tighter Schmidt-law relationship is found after smoothing to $1 \mathrm{kpc}$ resolution, which suggests that the spatial distribution of gas remains significantly influenced by the occurrence of star formation on scales larger than our resolution limit. By smoothing we better estimate the surface density at the location of the newly formed stars prior to their genesis. In Figure $15 b$ we have marked as yellow those points belonging to the $\mathrm{CO}$ ring. On the basis of the data in Figure $15 b$, the CO ring appears to exhibit a different regime in the local Schmidt law (flatter dependence on $\Sigma_{\text {gas }}$ ) compared to less conspicuous locations in the NGC 7331 gas distribution. We follow up on this in the next paragraph but first comment on the topic of the star formation threshold that appears so plainly in Figure 15b. In the context of a pixel-based Schmidt diagram, the local star formation threshold is evident as a shift to lower (undetected) values of $\Sigma_{\text {SFR }}$ which occurs suddenly at $0.7 \lesssim \log \Sigma_{\text {gas }} \lesssim 0.85$ in NGC 7331 . This range agrees well with the Schaye (2004) $0.1 Z_{\odot}$ prediction for the limiting column density $[\log N(\mathrm{H} \mathrm{I})=20.75$, equivalent to $\left.\log \Sigma_{\text {gas }}=0.77\right]$ at which the cool gaseous phase is expected to exist.

Wong \& Blitz (2002), Heyer et al. (2004), and Komugi et al. (2005) have demonstrated the improved quality of the Schmidtlaw relation on subgalactic scales that can be achieved by correlating 


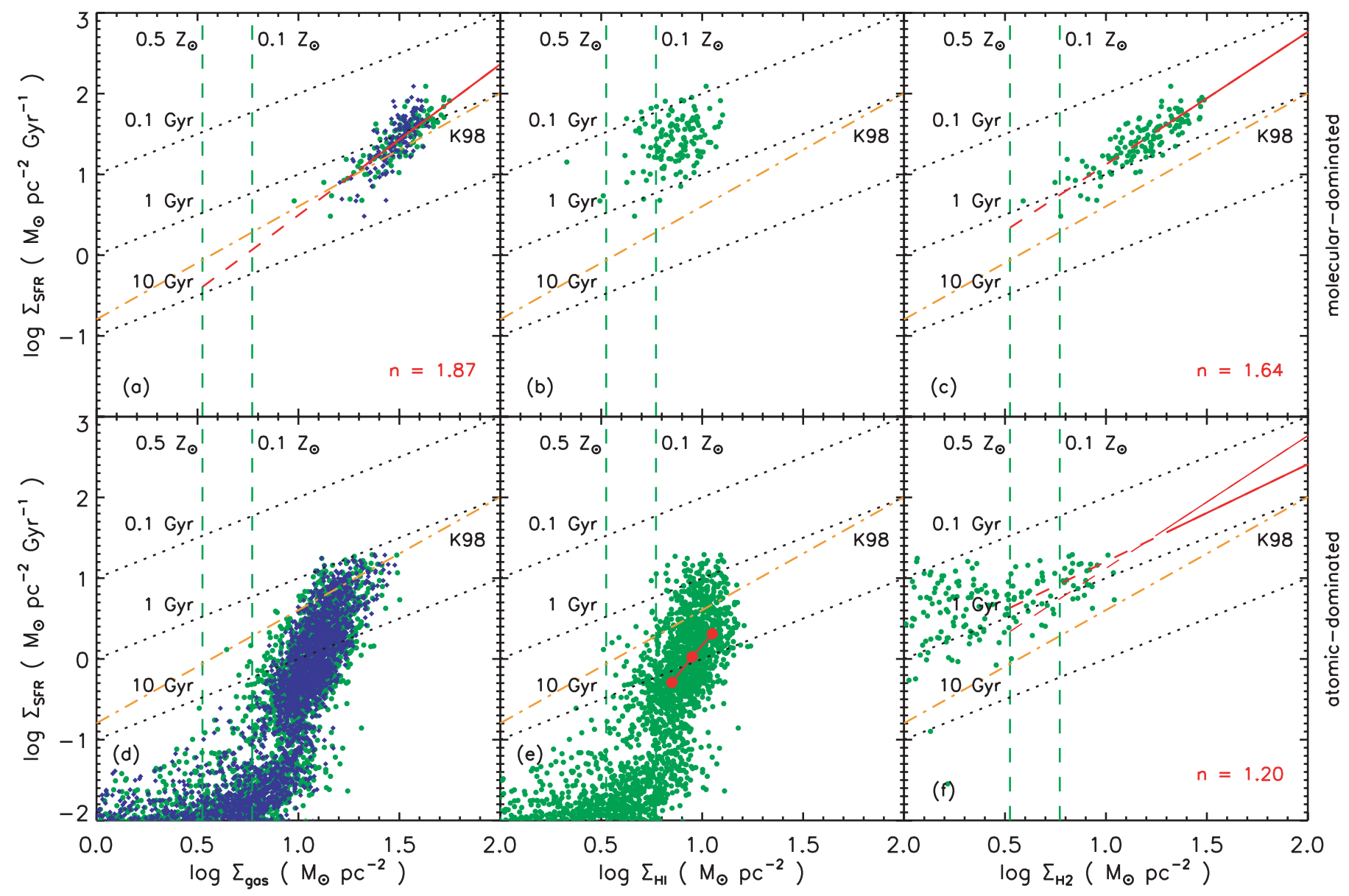

FIG. 16.-Local, extinction-free Schmidt law in NGC 7331 for molecular- and atomic-dominated regimes. (a) - (c) Our measurements for sight lines having $\Sigma_{\text {mol }} / \Sigma_{\text {atom }}>1 .(d)-(f) \Sigma_{\text {mol }} / \Sigma_{\text {atom }}<1$. Panels $(a)$ and $(d)$ present $\log \Sigma_{\mathrm{SFR}, \text { bol }}$ vs. $\log \Sigma_{\text {gas }}$ where the total gas distribution accounts for the atomic and molecular components traced by $\mathrm{H}$ i and $\mathrm{CO}$, respectively, and then scaled up to allow the contribution of He. The orange dash-dotted line, black dotted lines, and green dashed lines are as in Fig. 15. Panels $(b)$ and $(e)$ show $\log \Sigma_{\mathrm{SFR}, \text { bol }}$ Vs. $\log \Sigma_{\mathrm{H}_{\mathrm{I}}}$. Panels $(c)$ and $(f)$ illustrate the dependence of $\log \Sigma_{\mathrm{SFR}}$ on $\Sigma_{\mathrm{H}_{2}}$. In panels $(a)$ and $(c)$ the blue points show the effect of smoothing our observations to $1 \mathrm{kpc}$ resolution. The red lines in panels $(a),(c)$, and $(f)$ are linear fits to the data. The light red line in panel $(f)$ is simply a reproduction of the high-density molecular-only fit in panel (c).

the $\Sigma_{\mathrm{SFR}}$ against the total or even molecular-only gas surface density, rather than the atomic surface density. Heyer et al. (2004) showed that the correlation with molecular surface density was sustained even within locations dominated by atomic gas. Figure 16 shows the data from Figure $15 b$, as originally presented in terms of total gas surface density but also in atomic- and molecular-only forms. The data points have been segregated on the basis of the local $\Sigma_{\text {mol }} / \Sigma_{\text {atom }}$ value. We plot the two regimes, molecular-dominated $\left(\Sigma_{\text {mol }} / \Sigma_{\text {atom }}>1\right)$ and atomic-dominated $\left(\Sigma_{\text {mol }} / \Sigma_{\text {atom }}<1\right)$, in the top and bottom rows, respectively. For molecular-dominated regions, $\Sigma_{\mathrm{SFR}}$ follows a well-defined relation with both $\Sigma_{\text {gas }}$ (Fig. 16a) and $\Sigma_{\mathrm{H}_{2}}$ (Fig. 16c. However, $\Sigma_{\text {SFR }}$ and $\Sigma_{\mathrm{H}_{\mathrm{I}}}$ show no correlation (Fig. 16b). We plot the bestfitting power laws for Figures $16 a$ and $16 c$. In the moleculardominated environments being probed, the high-density end of the local Schmidt law is well represented as $\log \left(\Sigma_{\mathrm{SFR}}\right)=-1.37+$ $1.87 \log \left(\Sigma_{\text {gas }}\right)$ or $\log \left(\Sigma_{\mathrm{SFR}}\right)=-0.52+1.64 \log \left(\Sigma_{\mathrm{H}_{2}}\right)$. The expression cast in terms of total gas surface density is clearly only valid for $\log \left(\Sigma_{\text {gas }}\right)\left(M_{\odot} \mathrm{pc}^{-2}\right) \gtrsim 1.3$. To investigate the limits of application of the local Schmidt-law fit for molecular gas only, Figures $16 d-16 f$ examine the lower column density regimes, which tend to be predominately atomic. In particular, Figure $16 e$ shows that the surface density of $\mathrm{H}$ I and SFR are in fact correlated, although with a much steeper dependence than the powerlaw representations for total and molecular gas surface density in molecular-dominated locations. We attempted to obtain a bestfit representation of the correlation with $\Sigma_{\mathrm{H}_{\mathrm{I}}}$, but the distribution of data points is highly skewed to low $\Sigma_{\mathrm{SFR}}$, causing the fitting algorithm to become insensitive to the data points tracing higher $\Sigma_{\text {SFR }}$ environments. Instead of a fit, we plot the median value of $\log \Sigma_{\mathrm{SFR}}$ in three equally spaced bins of $\log \Sigma_{\mathrm{H}_{\mathrm{I}}}$ to demonstrate the systematic increase in $\log \Sigma_{\mathrm{SFR}}$ at progressively higher column densities. Figure $16 f$ illustrates that the relation between $\log \left(\Sigma_{\mathrm{H}_{2}}\right)$ and $\log \left(\Sigma_{\mathrm{SFR}}\right)$ in regions with predominately atomic gas is a continuation of the trend seen for molecular-dominated sight lines, although the data suggest a slightly smaller power-law index. Fitting over the entire range of surface densities above the $0.5 Z_{\odot}$ threshold yields a relation given by $\log \left(\Sigma_{\mathrm{SFR}}\right)\left(M_{\odot} \mathrm{pc}^{-2} \mathrm{Gyr}^{-1}\right)=0.0+1.20 \log \left(\Sigma_{\mathrm{H}_{2}}\right)$ $\left(M_{\odot} \mathrm{pc}^{-2}\right)$. The fact that the $\Sigma_{\mathrm{SFR}}-\Sigma_{\mathrm{H}_{2}}$ relation persists to low values of $\Sigma_{\mathrm{H}_{2}}$ with about the same slope as in high-density environments strengthens the claim that it is this relation which is fundamental and that the correlation with $\Sigma_{\mathrm{H}_{\mathrm{I}}}$ is merely a consequence of the increasing probability of $\mathrm{CO}$ formation in locations with copious $\mathrm{H}$ I. It would be of interest to isolate the component of $\mathrm{H}$ I emission associated with the cool neutral medium (CNM) and reexamine plots equivalent to Figures $16 b$ and $16 e$, rather than considering the aggregate emission from the warm neutral medium and CNM. Such analysis will be presented in a later paper. 


\section{CONCLUSIONS}

We have analyzed an extensive set of multiwavelength observations of NGC 7331, focusing on imagery from GALEX and Spitzer in the UV and IR, respectively. These data were complemented by radio, submillimeter, millimeter, and optical images. Our multiwavelength view of NGC 7331 revealed several important results concerning star formation and dust in this nearby, intermediate-type [SAB(rs)bcII] spiral.

1. The use of multiple tracers has allowed several independent measurements of the global SFR in NGC 7331 and on smaller scales $\left(0.4 \mathrm{kpc}\right.$, or $\left.6^{\prime \prime}\right)$ within prominent substructures. For the integrated SFR we find general agreement among the bolometric, IR, extinction-corrected UV, and extinction-corrected $\mathrm{H} \alpha$ estimates, placing the overall SFR in the range 4.4-6.3 $M_{\odot} \mathrm{yr}^{-1}$.

2. NGC 7331's morphology in the Spitzer $8 \mu \mathrm{m}$ band and at wavelengths up to at least $850 \mu \mathrm{m}$ is dominated by a central dust ring. This structure is a vigorous site of star formation (hosting approximately one-third of the total rate) but remains inconspicuous in our GALEX imagery. This is due to rather high extinction in the ring, up to $5 \mathrm{mag}$ at FUV for some of the IR-selected clumps, as determined by $L(\mathrm{IR}) / L(\mathrm{UV})$.

3. Radial-profile analysis has allowed determination of average dust properties, $\mathrm{SFH}$, and extinction as a function of position throughout NGC 7331. In $\S 4.3$, we demonstrate that changes in the dust SED are consistent with a preferentially "cooler" heating intensity distribution (see DH02) at large galactocentric distances. Based on the FIR/FUV determined in each radial bin, we measured the extinction at UV wavelengths, following Burgarella et al. (2005). A clear decrease in extinction with increasing radius was found, consistent with our results for resolved substructures. We subsequently dereddened the stellar SED with a Milky Way attenuation law, anchored by $A(\mathrm{FUV})$, prior to comparison with population-synthesis models. The UV-optical data were well matched by models having exponentially decreasing SFH. Our analysis suggests that the specific SFR is highest in the outer portion of the disk, despite the current activity in NGC 7331's dust ring.

4. We compared properties of UV-and UV+IR-selected substructures detected on $\sim 400 \mathrm{pc}$ scales in NGC 7331. For both samples we find that $L_{\mathrm{obs}}(\mathrm{FUV})$ and $L(24 \mu \mathrm{m})$ are essentially uncorrelated, perhaps reflecting unresolved complexity in these star-forming environments. The lack of a correlation, particularly for the UV+IR-selected sample, may be due to the effects of variable star-dust geometry and/or aging. Our photometry does demonstrate a correlation between $L(\mathrm{bol})$, derived from UV and IR measurements, and $L(\mathrm{IR}) / L_{\mathrm{obs}}(\mathrm{FUV})$, a measure of attenuation, as found by Calzetti et al. (2005) in M51. The power-law slope of this SFR-attenuation relation is equivalent to that first established by Heckman et al. (1998) for starburst galaxies. Translating our $L(\mathrm{IR}) / L_{\mathrm{obs}}(\mathrm{FUV})$ measurements into a UV extinction estimate for each substructure again demonstrated the radial decline in average extinction but showed that highly obscured star-forming regions can be found at any galactocentric distance and are not confined to the inner disk or the star-forming ring. We attempted to use the distribution of sources in the $\log \left[L(\right.$ IR $) / L_{\mathrm{obs}}$ (FUV) $]$ versus $\log \left[L_{\lambda, \text { obs }}(\mathrm{FUV}) / L_{\lambda, \text { obs }}(\mathrm{NUV})\right]$ plane to infer the most appropriate dust model for NGC 7331. Although the influence of burst age and more complex SFH renders this task difficult on a source-by-source basis, the overall distribution hints at Milky Way-type dust as might be anticipated given the other similarities between NGC 7331 and the Galaxy. A few of the most luminous star-forming structures appear to be exceptions to this and are better interpreted in the context of a starburst attenuation law.

5. We examine the nature of the local Schmidt law in NGC 7331, using both $\mathrm{H} \mathrm{I}$ and $\mathrm{CO}$ data to gauge the gas distribution and the bolometric estimator of SFR. Our analysis demonstrates that representation of the local star formation law in the form of azimuthal averages can lead to biased (high) values for the Schmidt-law index, as a result of neglecting nonlinearity in the underlying correlation due, for example, to the presence of a star formation threshold. In fact, we conducted a pixel-by-pixel Schmidt-law analysis which showed the clear presence of a star formation threshold and reduced star formation efficiency setting in at gas surface densities almost an order of magnitude higher than the threshold. Molecular- and atomic-dominated regions were then analyzed separately. We found that a power law of slope 1.64 (1.87) accurately described the Schmidt-law relation versus $\Sigma_{\mathrm{H}_{2}}\left(\Sigma_{\mathrm{gas}}\right)$ for molecular-dominated environments. The same locations showed no correlation between $\Sigma_{\mathrm{SFR}}$ and $\Sigma_{\mathrm{H}_{\mathrm{I}}}$. For atomicdominated regions, we found a trend for increasing $\Sigma_{\text {SFR }}$ at higher $\Sigma_{\mathrm{H}_{\mathrm{I}}}$, but the data were not well represented by a power law due to the highly skewed distribution of points (more at low values). We found that the Schmidt-law correlation with molecular-only surface density persisted in areas dominated by atomic gas, although with a possible change in slope.

GALEX (Galaxy Evolution Explorer) is a NASA Small Explorer, launched in 2003 April. We gratefully acknowledge NASA's support for construction, operation, and science analysis for the GALEX mission, developed in cooperation with the Centre National d'Etudes Spatiales of France and the Korean Ministry of Science and Technology. Support for this work, part of the Spitzer Space Telescope Legacy Science Program, was provided by NASA through contract 1224769 issued by the Jet Propulsion Laboratory (JPL), California Institute of Technology, under NASA contract 1407. This research has made use of the NASA/IPAC Extragalactic Database (NED) which is operated by JPL, California Institute of Technology, under contract with NASA. This research has made use of NASA's Astrophysics Data System.

Facilities: GALEX, Spitzer

\section{APPENDIX A}

\section{ESTIMATION OF THE STELLAR CONTRIBUTION TO $8 \mu \mathrm{m}$ EMISSION}

The following process was used to generate the model of stellar emission at $8 \mu \mathrm{m}$, assuming that the distribution of evolved stars contributing the most to the $8 \mu \mathrm{m}$ continuum are not responsible for producing PAH emission. First, convolution kernels (K. D. Gordon et al. 2008, in preparation) were used to match the 3.6 and $4.5 \mu \mathrm{m}$ images to the $8 \mu \mathrm{m}$ PSF. Many differently scaled versions of the PSFmatched $3.6 \mu \mathrm{m}$ image were then subtracted from the $8 \mu \mathrm{m}$ data, recording when the minimum correlation between the difference image and the scaled 3.6 $\mu \mathrm{m}$ image was obtained. We also demanded that no "holes" (negative dips) arise from continuum subtraction. The same method was used to determine a scale factor for the $4.5 \mu \mathrm{m}$ data relative to $8 \mu \mathrm{m}$. Finally, minor deviations from these nominal scale factors were allowed in order to maximize the agreement between the two trial $8 \mu \mathrm{m}$ dust-only images. The pair of scale factors that 
maximized such agreement was adopted and used to produce two nearly independent models of stellar emission. These models were averaged and subtracted from the observed $8 \mu \mathrm{m}$ image to obtain a stellar continuum-subtracted $8 \mu \mathrm{m}$ result. The scale factors used for 3.6 and $4.5 \mu \mathrm{m}$ were 0.37 and 0.64 , respectively, with the data in units of megajanskys per steradian. For comparison, the $3.6 \mu \mathrm{m}$ factor used by Helou et al. (2004), derived from an independent (population synthesis model-based) method, was 0.23 .

It should be noted that the 3.6 and $4.5 \mu \mathrm{m}$ bands also contain ISM emission at comparatively low levels, especially from $\mathrm{H}$ in regions (e.g., Helou et al. 2004), both in dust and gas emission (e.g., free-bound, H recombination). For truly safe stellar continuum removal, it would be ideal to use our method with $J H K$ imaging in place of the 3.6 and $4.5 \mu \mathrm{m}$ data. However, the 2MASS sensitivity was not suitable for this purpose except in the brightest parts of the galaxy. In any case, Regan et al. (2004) showed that dust emission contributes only $6 \%$ to the observed strength of the $4.5 \mu \mathrm{m}$ band. Consequently, the contribution of hot dust emission removed erroneously from our estimate of the $8 \mu \mathrm{m}$ dust image is minimal ( $<4 \%$ given the $4.5 \mu \mathrm{m}$ scale factor above) and does not significantly influence our analysis.

\section{APPENDIX B}

\section{ADOPTED CALIBRATION OF STAR FORMATION RATE TRACERS}

We used the recent calibration of Iglesias-Paramo et al. (2006), derived from Starburst99 (Leitherer et al. 1999) models assuming a Salpeter IMF $\left(0.1-100 M_{\odot}\right)$, solar metallicity, and a continuous starburst population. Taking $L($ bol $)=\nu_{\mathrm{FUV}} L_{\nu, \mathrm{obs}}(\mathrm{FUV})+(1-\eta) L(\mathrm{IR})$, the bolometric SFR estimate is given by

$$
\log \operatorname{SFR}(\mathrm{bol})\left(M_{\odot} \mathrm{yr}^{-1}\right)=\log L(\mathrm{bol})\left(L_{\odot}\right)-9.75 .
$$

The SFR computed from Spitzer data alone, SFR(IR), uses an identical calibration, after accounting for the fraction $(\eta)$ of dust luminosity associated with old stars. That is, we estimate SFR(IR) by replacing $L($ bol) with $(1-\eta) L($ IR). Following Bell (2003) and Iglesias-Paramo et al. (2006), we assume $\eta=0.32$ when calculating SFR globally and as a function of radius. This value of $\eta$ is comparable to the mean result (0.4) obtained by Hirashita et al. (2003) in a sample of "normal" (nonstarbursting) galaxies. However, for the small-scale structures of NGC 7331, we expect a negligible contribution of heating by older stellar populations. In this case, we take $\eta=0$ to better represent the starburst environment (Hirashita et al. 2003).

Starburst99 models also predict the following UV-based SFR relation,

$$
\log \operatorname{SFR}(\mathrm{FUV})\left(M_{\odot} \mathrm{yr}^{-1}\right)=\log L_{\text {corr }}(\mathrm{FUV})\left(L_{\odot}\right)-9.51,
$$

taken from Iglesias-Paramo et al. (2006). Note that the FUV luminosity in this expression must be dust corrected before use. We accomplish this following Buat et al. (2005) and Burgarella et al. (2005). They modeled the observed $L(\mathrm{IR}) / L_{\mathrm{obs}}(\mathrm{UV})$ using reddened population-synthesis models, deriving a polynomial fit for $A(\mathrm{FUV})$ and $A(\mathrm{NUV})$ as a function of $L(\mathrm{IR}) / L_{\mathrm{obs}}(\mathrm{FUV})$ and $L(\mathrm{IR}) /$ $L_{\mathrm{obs}}(\mathrm{NUV})$, respectively. Burgarella et al. (2005) confirmed the model relationship empirically using a sample of $\sim 200$ nearby galaxies. We adopt the formulation of Burgarella et al., which avoids negative $A$-values for very low $L(\mathrm{IR}) / L_{\mathrm{obs}}(\mathrm{UV})$ values. Buat et al. (2005) specifically showed that the calculation of $A$ from $L(\mathrm{IR}) / L_{\mathrm{obs}}(\mathrm{UV})$ is relatively insensitive to assumed SFH. Clearly, however, in order to provide a meaningful $A(\mathrm{FUV})$, within each source the stellar population heating the dust and producing the UV emission must be one in the same. This is justified for the galaxy as a whole (or large annular bins), but we also used this energy-balance technique to estimate $A(\mathrm{FUV})$ for each substructure in NGC 7331. In some cases on small scales, radiative energy transfer of UV light may occur, reducing the observed UV flux for a region and breaking the energy balance. However, this reduction is potentially countered by otherwise extraneous UV emission associated with non-star-forming (more evolved) stellar populations within a single aggregate source.

The breadth of our data set for NGC 7331 permits comparison of SFR metrics tracing the ISM as well as the stellar photospheric emission. Indirect measures such as recombination lines are widely used in the literature, most especially $\mathrm{H} \alpha$. It is important to note that these metrics are dependent not only on the energy flux from a stellar complex (and hence its SFH) but also the environment in which it is situated. Substantial uncertainty in the estimated SFRs for $\mathrm{H}$ iा complexes could be introduced if they are density bounded. Oey \& Kennicutt (1997) showed that up to $\sim 50 \%$ of the ionizing luminosity could be leaked to the surrounding diffuse medium, consistent with measurements of the $\mathrm{H} \alpha$ diffuse fraction. Integrating over diffuse and discrete components, we may use the following Starburst99 calibration from Hirashita et al. (2003):

$$
\mathrm{SFR}_{0}(\mathrm{H} \alpha)=7.89 \times 10^{-42} L(\mathrm{H} \alpha)\left(\mathrm{ergs} \mathrm{s}^{-1}\right) .
$$

The majority of $22 \mathrm{~cm}(1.4 \mathrm{GHz})$ continuum flux from a star-forming galaxy such as NGC 7331 is nonthermal synchrotron emission from relativistic electrons accelerated by supernovae. The lifetime of these high-energy electrons is approximately $100 \mathrm{Myr}$, making the monochromatic radio luminosity an indicator of the supernova rate (hence SFR) averaged over long timescales (similar to the UV). Schmitt et al. (2008) recalibrated the conversion from radio continuum luminosity to SFR, including nonthermal and thermal contributions to the observed radio SED. They followed the method of Condon \& Yin (1990) and Condon (1992) in predicting the spectral dependence and relative strength of these two components but used more up-to-date models (Starburst99) to link the supernova rate and SFR (a critical step in estimating the nonthermal contribution). At $\mathrm{L}$ band, in environments continuously forming massive stars, the flux density of synchrotron emission is about 7 times the thermal free-free emission from $\mathrm{H}$ in regions according to the Schmitt et al. (2008) recalibration. From Schmitt et al. (2008) we adopt the following relation for estimating the SFR from our WSRT observations:

$$
\mathrm{SFR}_{8}(1.4 \mathrm{GHz})=6.2 \times 10^{-29} L_{\nu}(1.4 \mathrm{GHz})\left(\mathrm{ergs} \mathrm{s}^{-1} \mathrm{~Hz}^{-1}\right) .
$$




\section{REFERENCES}

Alton, P. B., Lequeux, J., Bianchi, S., Churches, D., Davies, J., \& Combes, F. 2001, A\&A, 366, 451

Bell, E. F. 2002, ApJ, 577, 150 2003, ApJ, 586, 794

Bell, E. F., Gordon, K. D., Kennicutt, R. C., \& Zaritsky, D. 2002, ApJ, 565, 994

Bendo, G. J., et al. 2002, AJ, 123, 3067 2006, ApJ, 645, 134

Bianchi, L., et al. 2003, in The Local Group as an Astrophysical Laboratory, ed. M. Livio \& T. M. Brown (Cambridge: Cambridge Univ. Press), 10

Bianchi, S., Alton, P. B., Davies, J. I., \& Trewhella, M. 1998, MNRAS, 298, L49

Block, D. L., \& Puerari, I. 1999, A\&A, 342, 627

Boissier, S., Boselli, A., Buat, V., Donas, J., \& Milliard, B. 2004, A\&A, 424, 465

Boissier, S., \& Prantzos, N. 1999, MNRAS, 307, 857

Boissier, S., Prantzos, N., Boselli, A., \& Gavazzi, G. 2003, MNRAS, 346, 1215

Boissier, S., et al. 2005, ApJ, 619, L83 2007, ApJS, 173, 524

Boselli, A., Gavazzi, G., \& Sanvito, G. 2003, A\&A, 402, 37

Boselli, A., Lequeux, J., \& Gavazzi, G. 2002, A\&A, 384, 33

Bottema, R. 1999, A\&A, 348, 77

Braun, R., Oosterloo, T. A., Morganti, R., Klein, U., \& Beck, R. 2007, A\&A, 461, 455

Braun, R., Thilker, D., \& Walterbos, R. A. M. 2003, A\&A, 406, 829

Bruzual, G. \& Charlot, S. 2003, MNRAS, 344, 1000

Buat, V., \& Xu, C. 1996, A\&A, 306, 61

Buat, V., et al. 2005, ApJ, 619, L51

Burgarella, D., Buat, V., \& Iglesias-Páramo, J. 2005, MNRAS, 360, 1413

Burgarella, D., et al. 2006, A\&A, 450, 69

Calzetti, D. 2001, PASP, 113, 1449

Calzetti, D., Bohlin, R. C., Kinney, A. L., Storchi-Bergmann, T., \& Heckman, T. M. 1995, ApJ, 443, 136

Calzetti, D., Kinney, A. L., \& Storchi-Bergmann, T. 1994, ApJ, 429, 582

Calzetti, D., et al. 2005, ApJ, 633, 871

Chabrier, G. 2003, PASP, 115, 763

Condon, J. J. 1992, ARA\&A, 30, 575

Condon, J. J., \& Yin, Q. F. 1990, ApJ, 357, 97

Cowan, J. J., Romanishin, W., \& Branch, D. 1994, ApJ, 436, L139

Dale, D. A., \& Helou, G. 2002, ApJ, 576, 159 (DH02)

Dale, D. A., et al. 2000, AJ, 120, 583

de Vaucouleurs, G., \& Pence, W. D. 1978, AJ, 83, 1163

Dopita, M. A., \& Ryder, S. D. 1994, ApJ, 430, 163

Dutil, Y., \& Roy, J.-R. 1999, ApJ, 516, 62

Elmegreen, B. G. 2002, ApJ, 577, 206

Engelbracht, C. W., Gordon, K. D., Rieke, G. H., Werner, M. W., Dale, D. A., \& Latter, W. B. 2005, ApJ, 628, L29

Engelbracht, C. W., et al. 2006, ApJ, 642, L127

Fazio, G. G., et al. 2004, ApJS, 154, 10

Filho, M. E., Barthel, P. D., \& Ho, L. C. 2002, ApJS, 142, 223

Freedman, W. L., et al. 2001, ApJ, 553, 47

García-Gómez, C., Athanassoula, E., \& Barberà, C. 2002, A\&A, 389, 68

Gil de Paz, A., et al. 2007, ApJS, 173, 185

Goldader, J. D., Meurer, G., Heckman, T. M., Seibert, M., Sanders, D. B., Calzetti, D., \& Steidel, C. C. 2002, ApJ, 568, 651

Gordon, K. D., et al. 2005, PASP, 117, 503

Haynes, M. P., van Zee, L., Hogg, D. E., Roberts, M. S., \& Maddalena, R. J. 1998, AJ, 115, 62

Heckman, T. M., Robert, C., Leitherer, C., Garnett, D. R., \& van der Rydt, F. 1998, ApJ, 503, 646

Helfer, T. T., Thornley, M. D., Regan, M. W., Wong, T., Sheth, K., Vogel, S. N., Blitz, L., \& Bock, D. C.-J. 2003, ApJS, 145, 259

Helou, G., et al. 2004, ApJS, 154, 253

Heyer, M. H., Corbelli, E., Schneider, S. E., \& Young, J. S. 2004, ApJ, 602 , 723

Hirashita, H., Buat, V., \& Inoue, A. K. 2003, A\&A, 410, 83

Holwerda, B. W., Gonzalez, R. A., Allen, R. J., \& van der Kruit, P. C. 2005a, AJ, 129, 1396

. 2005b, A\&A, 444, 101
Holwerda, B. W., González, R. A., Allen, R. J., \& van der Kruit, P. C. 2004, in IAU Symp. 220, Dark Matter in Galaxies, ed. S. D. Ryder et al. (San Francisco: ASP), 323

Hoopes, C. G., \& Walterbos, R. A. M. 2003, ApJ, 586, 902

Hoopes, C. G., et al. 2005, ApJ, 619, L99 2007, ApJS, 173, 441

Iglesias-Paramo, J., et al. 2006, ApJS, 164, 38

Jarrett, T. H., Chester, T., Cutri, R., Schneider, S. E., \& Huchra, J. P. 2003, AJ, 125,525

Kennicutt, R. C. 1983, ApJ, 272, 54

. 1989, ApJ, 344, 685

. 1998a, ApJ, 498, 541

1998b, ARA\&A, 36, 189

Kennicutt, R. C., et al. 2003, PASP, 115, 928

Komugi, S., Sofue, Y., Nakanishi, H., Onodera, S., \& Egusa, F. 2005, PASJ, 57, 733

Kong, X., Charlot, S., Brinchmann, J., \& Fall, S. M. 2004, MNRAS, 349, 769

Kravtsov, A. V. 2003, ApJ, 590, L1

Kravtsov, A. V., Gnedin, O. Y., \& Klypin, A. A. 2004, ApJ, 609, 482

Krumholz, M. R., \& McKee, C. F. 2005, ApJ, 630, 250

Leitherer, C., et al. 1999, ApJS, 123, 3

Li, Y., Mac Low, M.-M., \& Klessen, R. S. 2006, ApJ, 639, 879

Mac Low, M.-M., \& Klessen, R. S. 2004, Rev. Mod. Phys., 76, 125

Martin, D. C., et al. 2005, ApJ, 619, L1

McGaugh, S. S. 2005, ApJ, 632, 859

Meurer, G. R., Heckman, T. M., \& Calzetti, D. 1999, ApJ, 521, 64

Morrissey, P., et al. 2005, ApJ, 619, L7

Muñoz-Mateos, J. C., Gil de Paz, A., Boissier, S., Zamorano, J., Jarrett, T., Gallego, J., \& Madore, B. F. 2007, ApJ, 658, 1006

Nakanishi, H., \& Sofue, Y. 2003, PASJ, 55, 191

Nussbaumer, H., \& Rusca, C. 1979, A\&A, 72, 129

Oey, M. S. 2004, Ap\&SS, 289, 269

Oey, M. S., \& Kennicutt, R. C. 1997, MNRAS, 291, 827

Petit, H. 1998, A\&AS, 131, 317

Popescu, C. C., Tuffs, R. J., Völk, H. J., Pierini, D., \& Madore, B. F. 2002, ApJ, 567, 221

Popescu, C. C., et al. 2005, ApJ, 619, L75

Prada, F., Gutierrez, C. M., Peletier, R. F., \& McKeith, C. D. 1996, ApJ, 463, L9

Regan, M. W., Thornley, M. D., Helfer, T. T., Sheth, K., Wong, T., Vogel, S. N., Blitz, L., \& Bock, D. C.-J. 2001, ApJ, 561, 218

Regan, M. W., et al. 2004, ApJS, 154, 204

Rieke, G. H., et al. 2004, ApJS, 154, 25

Roussel, H., Gil de Paz, A., Seibert, M., Helou, G., Madore, B. F., \& Martin, C. 2005, ApJ, 632, 227

Rownd, B. K., \& Young, J. S. 1999, AJ, 118, 670

Sanders, D. B., \& Mirabel, I. F. 1996, ARA\&A, 34, 749

Sanders, D. B., Soifer, B. T., Elias, J. H., Madore, B. F., Matthews, K.,

Neugebauer, G., \& Scoville, N. Z. 1988, ApJ, 325, 74

Sanduleak, N. 1969, AJ, 74, 47

Schaye, J. 2004, ApJ, 609, 667

Schlegel, D. J., Finkbeiner, D. P., \& Davis, M. 1998, ApJ, 500, 525

Schmidt, M. 1959, ApJ, 129, 243

Schmitt, H., et al. 2008, ApJ, in press

Seibert, M., et al. 2005, ApJ, 619, L23

Smith, J. D. T., et al. 2004, ApJS, 154, 199

Soifer, B. T., Sanders, D. B., Madore, B. F., Neugebauer, G., Danielson, G. E., Elias, J. H., Lonsdale, C. J., \& Rice, W. L. 1987, ApJ, 320, 238

Telesco, C. M., Gatley, I., \& Stewart, J. M. 1982, ApJ, 263, L13

Thilker, D. A., Braun, R., \& Walterbos, R. A. M. 2000, AJ, 120, 3070

Tuffs, R. J., et al. 2002, ApJS, 139, 37

Walter, F., Brinks, E., de Blok, W. J. G., Thornley, M. D., \& Kennicutt, R. C.

2005, in ASP Conf. Ser. 331, Extra-Planar Gas, ed. R. Braun (San Francisco: ASP), 269

Werner, M. W., et al. 2004, ApJS, 154, 1

Wong, T., \& Blitz, L. 2002, ApJ, 569, 157

Young, J. S., \& Scoville, N. 1982, ApJ, 260, L41 